\title{
1 Activation of a signaling pathway by the physical translocation of a 2 chromosome
}

6 Mathilde Guzzo ${ }^{1}$, Allen G. Sanderlin ${ }^{1,2}$, Lennice K. Castro ${ }^{1}$, Michael T. Laub ${ }^{1,2, \#}$

$12{ }^{1}$ Department of Biology, Massachusetts Institute of Technology, Cambridge, MA 02139

$13{ }^{2}$ Howard Hughes Medical Institute, Massachusetts Institute of Technology, Cambridge, MA $14 \quad 02139$

18 \# corresponding author: laub@mit.edu 


\section{Abstract}

21 In every organism, the cell cycle requires the execution of multiple cellular processes in a strictly 22 defined order. However, the mechanisms used to ensure such order remain poorly understood, 23 particularly in bacteria. Here, we show that the activation of the essential CtrA signaling pathway 24 that triggers cell division in Caulobacter crescentus is intrinsically coupled to the successful 25 initiation of DNA replication via the physical translocation of a newly-replicated chromosome, 26 powered by the ParABS system. We demonstrate that ParA accumulation at the new cell pole 27 during chromosome segregation recruits ChpT, an intermediate component of the CtrA signaling 28 pathway. ChpT is normally restricted from accessing the selective PopZ polar microdomain until 29 the new chromosome and ParA arrive. Consequently, any disruption to DNA replication initiation 30 prevents the recruitment of ChpT and, in turn, cell division. Collectively, our findings reveal how 31 major cell-cycle events are coordinated in Caulobacter and, importantly, how the physical 32 translocation of a chromosome triggers an essential signaling pathway. 


\section{Introduction}

34 In all domains of life, cells must ensure that DNA replication, chromosome segregation, and cell division occur in the right order. A failure to coordinate these cell-cycle processes can lead to genome instability or cell death (Storchova and Pellman, 2004). In eukaryotes, numerous checkpoint systems ensure that each step of the cell cycle has completed before proceeding to the next one (Hartwell and Weinert, 1989; Murray, 1992). Few bona fide checkpoints have been identified in bacteria (Rudner and Losick, 2001) and the mechanisms that ensure orderly progression through the bacterial cell cycle remain largely unknown.

41 The $\alpha$-proteobacterium Caulobacter crescentus has a tightly regulated cell cycle with DNA 42 replication occurring only once per cell cycle under all growth conditions (Marczynski, 1999). C. 43 crescentus also features an asymmetric cell division that generates two distinct daughter cells: a sessile stalked cell that immediately initiates replication and a motile swarmer cell that cannot initiate replication until it differentiates into a stalked cell (Fig. 1A). The initiation of DNA replication requires (i) the inactivation of CtrA, a response regulator that can directly silence the origin of replication (Domian et al., 1997; Quon et al., 1998), and (ii) accumulation of ATP-bound DnaA, the conserved replication initiator protein in bacteria (Gorbatyuk and Marczynski, 2001; Fernandez-Fernandez et al., 2011; Jonas et al., 2011). CtrA must then be produced de novo and activated by phosphorylation after DNA replication has initiated. In predivisional cells, CtrA directly promotes the expression of nearly 100 target genes critical to many cellular processes, including cell division (Laub et al., 2000, 2002). If DNA replication is blocked, CtrA is not produced (Wortinger et al., 2000) and cell division does not occur (Iniesta et al., 2010b), but the mechanism responsible for coupling replication to CtrA activation has not been identified.

CtrA activity is controlled by a phosphorelay from the histidine kinase CckA to the histidine phosphotransferase ChpT to CtrA (Biondi et al., 2006) (Fig. S1A). Following DNA replication, CckA is recruited to the nascent swarmer pole of predivisional cells where its kinase activity is stimulated by the atypical histidine kinase DivL (Jacobs et al., 1999; Chen et al., 2009; Angelastro et al., 2010; Iniesta et al., 2010a; Tsokos et al., 2011) (Fig. 1A, S1A). CckA and ChpT also drive phosphorylation of CpdR to prevent it from promoting CtrA degradation (Jenal and Fuchs, 1998; Biondi et al., 2006; Iniesta et al., 2006; Chien et al., 2007) (Fig. S1A). The localization of CckA and DivL to the swarmer pole and CckA kinase activity depend on DNA replication initiation

63 (Iniesta et al., 2010b), but the underlying mechanism is not known. Additionally, whether the 64 localization of CckA and DivL is the key means of coupling DNA replication to CtrA activation and 65 subsequent cell division has not been established. 
Here, we show that swarmer pole localization of CckA and DivL can be restored in the absence of DNA replication by ectopically producing the scaffold protein PodJ. Surprisingly though, this polarization of CckA and DivL is not sufficient to activate CtrA. Instead, we find that the activation of CtrA after DNA replication has initiated depends on the recruitment of the intermediate component of the phosphorelay, ChpT, to the swarmer pole by the chromosome segregation machinery. Replication initiation occurs near the stalked pole in Caulobacter. One of the duplicated chromosomes is then translocated to the opposite cell pole via a complex interplay between the nucleoid-bound ATPase ParA and the protein ParB bound to origin-proximal parS sites (Toro et al., 2008; Lim et al., 2014). We show that the accumulation of nucleoid-free ParA at the swarmer pole once chromosome translocation has completed drives the recruitment of ChpT, thereby completing the phosphorelay from CckA to CtrA. When DNA replication initiation is blocked or chromosome translocation is disrupted, ChpT fails to accumulate at the swarmer pole, preventing cell division. Thus, our work now reveals the molecular basis of a substrate-product relationship, rather than a checkpoint, by which replication and cell division are coupled in Caulobacter crescentus. More broadly, our results also demonstrate how a physical cue, in this case the translocation of a chromosome, can serve as the activating signal for a complex signal transduction pathway.

\section{Results}

\section{CtrA activation in predivisional cells depends on DNA replication}

85 Prior studies have indicated that CtrA activation in predivisional cells depends on DNA replication initiation (Wortinger et al., 2000; Iniesta et al., 2010b). To corroborate this observation and establish a genetic system for further studying it, we used a strain in which the endogenous $d n a A$ promoter was replaced by the E. coli lac promoter and the lacl gene was integrated at the $h f a$ locus (Badrinarayanan et al., 2015). We grew cells in the absence of IPTG for 90 minutes to deplete DnaA, and then synchronized and released cells into fresh minimal medium (M2G+) with or without IPTG to induce dnaA expression or not, respectively. In the presence of DnaA, DNA replication initiated within 40 min., as judged by flow cytometry (Fig. S1B), whereas in DnaA-

93 depleted cells, DNA replication had not initiated even by the end of the time-course (120 min.)

94 (Gorbatyuk and Marczynski, 2001) (Fig. S1B).

To test whether CtrA was activated in the absence of DNA replication, we first used Western blots 
accumulation (Quon et al., 1998). In cells producing DnaA, CtrA accumulated in late predivisional cells, $\sim 120$ min. post-synchronization, as expected (Fig. 1B), but in cells lacking DnaA, CtrA did not accumulate (Fig. 1B) because ctrA is not transcribed in cells that lack DnaA (Holtzendorff et al., 2004). Therefore, we ectopically expressed ctrA from a xylose-inducible promoter on a plasmid in cells also depleted of DnaA, but CtrA still did not accumulate by 120 min. postsynchronization in the presence of xylose, presumably because it gets degraded (Fig. 1B).

104 The lack of CtrA accumulation in cells depleted of DnaA supports the notion that CtrA activation 105 is dependent on DNA replication initiation. However, cells lacking DnaA could be competent for 106 CtrA phosphorylation, but also be actively degrading CtrA. To rule out this possibility, we expressed a constitutively stable variant, $\operatorname{ct} A \Delta 3 \Omega$ that can still be regulated by phosphorylation (Domian et al., 1997) (Fig. S1C), in cells depleted of DnaA. We now observed an accumulation of this stabilized CtrA (Fig. S1D). To assess CtrA phosphorylation, we used qRT-PCR to measure mRNA levels of the CtrA target gene divK. In cells expressing dnaA, divK mRNA levels increased substantially in predivisional cells (Fig. 1C). In contrast, for cells depleted of DnaA, divK expression remained low throughout the cell cycle either with or without $\operatorname{Ctr} A \triangle 3 \Omega$ being produced

113 (Fig. 1C). Thus, even if abundant in cells, CtrA is not phosphorylated in the absence of DnaA and 114 DNA replication initiation.

115 Known regulators cannot account for a failure to activate CtrA after inhibiting DNA 116 replication

117 DnaA promotes the transcription of gcrA (Hottes et al., 2005), a key transcriptional regulator in 118 Caulobacter (Haakonsen et al., 2015). Thus, in the absence of DnaA, either GcrA or an unknown 119 GcrA-regulated factor that is required for CtrA activation could be missing. To test this possibility, 120 we ectopically expressed gcrA-3xflag (Fig. S1E) and ctrA in cells depleted of DnaA. However, 121 CtrA did not accumulate in predivisional cells of this strain (Fig. S1E), and divK was not activated 122 even when expressing non-degradable $\operatorname{CtrA} \Delta 3 \Omega$ (Fig. S1F). These results indicate that a lack of 123 GcrA in the absence of DnaA is not responsible for blocking CtrA activation.

124 Next, we considered whether inhibiting DNA replication initiation somehow inactivated CckA, 125 which can be directly inhibited by c-di-GMP (Lori et al., 2015). To test this model, we reduced c126 di-GMP levels in cells depleted of DnaA by expressing a phosphodiesterase (PA5295) from 127 Pseudomonas aeruginosa (Abel et al., 2013). In this strain, low c-di-GMP levels prevented CtrA 128 degradation at the swarmer-to-stalk cell transition, so CtrA remained present throughout the cell 129 cycle (Fig. S1G). However, divK expression was not upregulated in the absence of DnaA when 
expressing this phosphodiesterase (Fig. S1H). Thus, we infer that blocking DNA replication does not prevent CtrA activation through c-di-GMP-dependent inhibition of CckA.

132 Finally, we tested whether SciP, a protein that inhibits CtrA transcriptional activity in swarmer cells

133 (Gora et al., 2010), somehow accumulated following a block to replication initiation. However, 134 SciP was still properly degraded $30 \mathrm{~min}$. after synchronization and remained low in cells depleted 135 of $d n a A$ (Fig. S1I), indicating that an accumulation of SciP does not explain the inhibition of CtrA 136 if DNA replication fails to initiate.

\section{Polar localization of DivL and CckA is not sufficient to activate CtrA following a block to} 138 DNA replication

139 CtrA phosphorylation in predivisional cells depends on the polar localization of the histidine 140 kinases CckA and DivL (Jacobs et al., 1999; Chen et al., 2009; Angelastro et al., 2010; Iniesta et al., 2010a; Tsokos et al., 2011). This localization of CckA and DivL depends on DNA replication (Iniesta et al., 2010b), and we confirmed that in the absence of DnaA, CckA-GFP and DivL-GFP do not localize to the nascent swarmer pole of predivisional cells (Fig. 1D-F). We then wanted to test if this defect in CckA and DivL localization was the limiting step for CtrA activation in the absence of DnaA. Notably, DnaA directly upregulates podJ expression after replication initiation (Hottes et al., 2005) (Fig. S1C) and the polar localization of DivL and CckA both depend on PodJ (Curtis et al., 2012). Thus, to test if PodJ was the limiting factor for CckA and DivL localization (and ultimately for CtrA activation) in the absence of DnaA, we ectopically expressed podJ using a cumate-inducible promoter (Kaczmarczyk et al., 2013) in the dnaA depletion strain (Fig. S1C). Inducing PodJ was sufficient to restore CckA-GFP and DivL-GFP to the nascent swarmer pole in the absence of DnaA (Fig. 1D-F; -dnaA + podJ condition). To test if CtrA activation was restored, we took cells depleted of DnaA and ectopically expressed ctrA and podJ. CtrA levels remained low even $120 \mathrm{~min}$. after synchronization (Fig. 1B) and divK expression was not upregulated, even when expressing the $c t r A \Delta 3 \Omega$ stable variant (Fig. 1C). These results indicate that PodJ production is sufficient to polarize CckA and DivL in the absence of DNA replication, but that CtrA is still not phosphorylated.

157 Importantly, the phosphorylation of CtrA requires autophosphorylated CckA to transfer a 158 phosphoryl group to the histidine phosphotransferase ChpT which then serves as the direct 159 phosphodonor to CtrA (Biondi et al., 2006) (Fig. S1A). ChpT was not considered in previous 160 studies of how DNA replication initiation affects CtrA. To test whether ChpT localization depends 161 on replication initiation, we engineered the DnaA-depletion strain to produce ChpT-YFP from its 162 native genomic locus. In the presence of DnaA, ChpT-YFP foci were seen at the nascent swarmer 
pole of predivisional cells (Fig. 1D,G). In contrast, for cells depleted of DnaA, no foci were seen at that pole, only the stalked pole (Fig. 1D,G). Thus, like CckA and DivL, ChpT localization to the nascent swarmer pole depends on the successful initiation of DNA replication. However, in sharp contrast to our observations for CckA and DivL, the ectopic production of PodJ was not sufficient to localize ChpT-YFP (Fig. 1D,G). Thus, ChpT localization to the swarmer pole is not determined simply by the localization of CckA. Additionally, this finding indicates that localization of ChpT may be the limiting step in coupling CtrA activation to DNA replication initiation.

\section{Only partial replication of the chromosome is required for CtrA activation}

171 The findings presented thus far suggest that the requirement for DnaA in localizing ChpT to the 172 swarmer pole, and in turn activating CtrA, is not related to its role as a transcription factor. Thus, 173 we favored the possibility that something about the act of DNA replication itself, which is triggered 174 by DnaA, is required to activate CtrA. To further explore how replication controls CtrA activation, 175 we examined a strain in which wild-type DnaA was depleted but ectopically produces $176 \operatorname{DnaA}(\mathrm{R} 357 \mathrm{~A})$, which is likely locked in the active, ATP-bound form (Fernandez-Fernandez et al., 177 2011; Jonas et al., 2011). This strain overinitiates DNA replication and the chromosome content 178 per cell exceeds $2 \mathrm{~N}$, as judged by flow cytometry (Fig. S2A). Using qPCR at seven loci along the chromosome (Fig. 2A), we found that cells producing DnaA(R357A), accumulated up to 5 copies of genomic regions near the origin (Fig. 2A). In contrast, cells producing wild-type DnaA had at most 2 copies, as expected for wild-type Caulobacter (Marczynski, 1999) (Fig. 2A). Despite the high initiation rate of cells producing DnaA(R357A), the genomic copy number was less than 2 at $0.67 \mathrm{Mb}$ and unreplicated beyond $1 \mathrm{Mb}$ (Fig. 2A). Thus, this strain enabled us to ask whether only partial $(\sim 1 / 3)$ replication of the chromosome was sufficient to activate CtrA.

We first examined ctrA transcription, finding that it remained relatively low in cells producing DnaA(R357A) (Fig. S2B) despite the accumulation of GcrA (Fig. S2C), possibly because the ctrA locus at $0.764 \mathrm{Mb}$ remains fully methylated, which reduces its transcription (Holtzendorff et al., 2004). We therefore placed ctrA under the control of a vanillate-inducible promoter in cells also engineered to deplete wild-type DnaA and produce DnaA(R357A). We depleted DnaA for 90 minutes, synchronized cells, and released them into a medium that enables repression of wildtype DnaA and induction of DnaA(R357A) and ctrA (Fig. S2D). CtrA now strongly accumulated in

192 late predivisional cells despite the absence of full chromosome replication (Fig. 2B). Additionally, 193 the CtrA-regulated genes $\operatorname{divK}$ and $p d e A$ were strongly upregulated in cells expressing both $194 d n a A(R 357 A)$ and ctrA compared to cells depleted of DnaA (Fig. 2C). We conclude that replication 
of the entire chromosome is not required for CtrA activation. Either the act of replication initiation itself or an event coupled to the earliest stages of replication is required.

197 Disrupting chromosome segregation prevents CtrA activation

198 Our experiments with the overinitiating $\operatorname{dnaA}(R 357 A)$ strain suggested that replication of the first 199 third of the chromosome is sufficient to trigger CtrA activation in predivisional cells (Fig. 2). 200 Notably, chromosome segregation is initiated upon replication of the parS site, which is located 201 just $8 \mathrm{~kb}$-away from oriC, the chromosomal origin of replication (Toro et al., 2008). After the parS 202 locus is duplicated, one of the copies separates away from the stalked pole, and then ParA and 203 ParB drive its rapid translocation across the cell to the nascent swarmer pole (Ptacin et al., 2010; 204 Schofield et al., 2010; Shebelut et al., 2010; Lim et al., 2014) (Fig. 3A). Thus, we decided to 205 investigate whether segregation of the chromosome is a critical step in CtrA activation. Such a 206 role for chromosome segregation was previously dismissed (Iniesta et al., 2010b) because 207 synthesizing ParA(K20R), a variant of ParA that blocks chromosome segregation without altering 208 replication (Toro et al., 2008) (Fig. S3A), did not affect CckA-GFP localization. However, the 209 impact of ParA(K20R) on CtrA activation and ChpT localization was not tested.

210 We first tested if disrupting chromosome segregation by ectopically expressing parA(K20R) would 211 affect CtrA activation. To try and maximize the disruption of chromosome segregation but retain 212 synchronizability of cells, we induced parA(K20R) 60 minutes before synchronizing cells (Fig. 3B$213 \mathrm{C})$. We found that the expression levels of two CtrA-activated genes, $\operatorname{divK}$ and $p d e A$, and CtrA 214 protein levels were both modestly, but significantly reduced in predivisional cells (100 min. post215 synchronization) expressing $\operatorname{parA}(K 20 R)$ compared to the non-induced condition (Fig. 3B-C).

216 These results indicated that improper segregation of the chromosome impacts CtrA activation. $217 \operatorname{ParA}(\mathrm{K} 20 \mathrm{R})$ did not completely eliminate CtrA activation, likely due to the heterogeneity of $218 \operatorname{ParA}(\mathrm{K} 20 \mathrm{R})$ accumulation and an incomplete disruption of chromosome segregation, which we 219 return to later.

220 To corroborate the results with $\operatorname{ParA}(K 20 R)$, we sought to perturb chromosome segregation, 221 again without disrupting DNA replication, in an alternative way. To do this, we overexpressed the 222 spreading-deficient mutant parB(G101S) (Tran et al., 2018), which contains a point mutation in 223 the arginine-rich patch important for CTP binding (Jalal et al., 2020). Inducing parB(G101S) does 224 not affect DNA replication (Fig. S3B), but prevents chromosome segregation, as indicated by a 225 failure to segregate the ParB-associated protein MipZ to the nascent swarmer pole (Fig. S3C). 226 As with $\operatorname{parA}(K 20 R)$, inducing the expression of $\operatorname{parB}(\mathrm{G} 101 S) 60$ minutes before synchronization 227 also modestly, but significantly, reduced the expression levels of two CtrA-regulated genes, divK 
228 and $p d e A$, and CtrA protein levels (Fig. 3D-E). Additionally, we observed that inducing

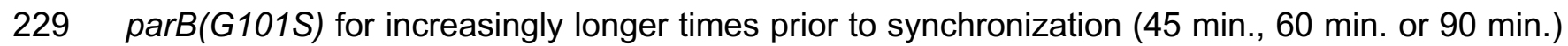
230 enhanced the effect on CtrA activation (Fig. 3F), suggesting that the reduction in CtrA activation

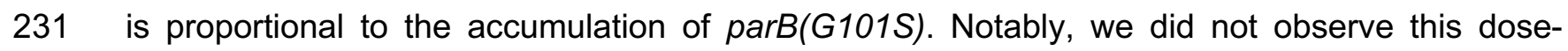
232 response effect when expressing wild-type parB instead of parB(G101S) (Fig. 3F). Taken 233 together, our results suggest that chromosome segregation is critical in triggering the proper 234 accumulation of phosphorylated, active CtrA following DNA replication initiation.

235 Disrupting chromosome segregation affects the localization of ChpT, but not CckA and 236 DivL

237 To investigate the connection between chromosome translocation and the polarization of DivL, 238 CckA, and ChpT, we induced parA(K20R) from a vanillate-inducible promoter in cells also 239 expressing MipZ-CFP, CckA-GFP, DivL-GFP, or ChpT-sfGFP at their native genomic loci. When 240 inducing $\operatorname{parA}(K 20 R)$ for $30 \mathrm{~min}$. before synchronization, some cells can divide while some cannot 241 and instead become elongated. The percentage of elongated cells when expressing $\operatorname{par} A(K 20 R)$ 242 was 38\% for MipZ-CFP ( $n=309), 50 \%$ for CckA-GFP ( $n=335), 36 \%$ for DivL-GFP $(n=207)$ and $24335 \%$ for ChpT-sfGFP ( $n=310)$. This heterogeneity likely reflects heterogeneity in ParA(K20R) 244 accumulation, with elongated, undivided cells representing those with enough $\operatorname{ParA}(K 20 \mathrm{R})$ to 245 disrupt chromosome segregation. We therefore focused on elongated cells that had not divided 246 by $270 \mathrm{~min}$. after synchronization in the following time-lapse experiments; for reference, non247 induced cells handled identically divide after 150 min. (Fig. 4A). In the elongated cells, MipZ248 CFP (which binds and labels ParB-parS complexes (Thanbichler and Shapiro, 2006)) localization 249 to the new pole was strongly reduced, as expected if chromosome segregation is disrupted (Toro 250 et al., 2008) (Fig. 4B-C). For both dividing and elongating cells, CckA-GFP and DivL-GFP 251 accumulated at the new swarmer pole in almost all cells of the population (Fig. 4B, D, S4A) 252 (Iniesta et al., 2010b), consistent with our findings that CckA and DivL localization depends 253 primarily on PodJ accumulation at the pole (Fig. 1D-F), not DNA replication or subsequent 254 chromosome segregation. In sharp contrast to CckA and DivL, only $30 \%$ of elongated cells 255 producing ParA(K20R) had detectable ChpT-sfGFP foci at the nascent swarmer pole (Fig. 4B, E). 256 These results suggest that, unlike CckA and DivL, ChpT localization to the swarmer pole depends 257 on proper chromosome segregation.

258 We also examined ChpT-sfGFP localization in cells overexpressing a wild-type copy of parA (Fig. 259 4F-G), which does not prevent chromosome translocation as with parA(K20R) (Fig. 4G, S4B). In 260 these cells, ChpT-sfGFP now accumulated at the nascent swarmer pole (Fig. 4F-G). In fact, 
whereas ChpT-sfGFP localization to that pole is typically transient in WT cells (Fig. 4A) and not always easily detectable (Fig. 4B), overexpression of wild-type parA strongly increased the stability and detectability of ChpT-sfGFP at the new pole in most of the cells in the population (Fig. 4F-G), and resembled MipZ-CFP localization under the same conditions (compare Fig. 4F and S4B). Collectively, our results support the conclusion that localization of ChpT to the swarmer pole, and subsequent activation of CtrA, depends on successful chromosome translocation, which occurs shortly after replication initiation.

ChpT localization to the new pole and access to the PopZ microdomain depends on ParA

269 For ChpT-sfGFP to localize to the swarmer pole, it must access the microdomain formed by the 270 polar organizer protein PopZ, which excludes most cytoplasmic proteins (Ebersbach et al., 2008; 271 Bowman et al., 2010) unless they bind a component of the microdomain (Lasker et al., 2020).

272 The formation of the PopZ microdomain was not affected by the overexpression of $\operatorname{parA}(K 20 R)$ 273 (Laloux and Jacobs-Wagner, 2013) (Fig. S4C), and ChpT-sfGFP appeared confined to the rest 274 of the cell (Fig. 4E) suggesting that ChpT is excluded from the PopZ microdomain in the absence 275 of chromosome translocation. Overexpressing popZ can expand the PopZ microdomain, especially at the old cell pole (Ebersbach et al., 2008; Bowman et al., 2010; Lasker et al., 2020). We induced the expression of popZ after synchronization (of cells also producing ParA(K20R) to disrupt chromosome segregation) and asked how this affected CckA and ChpT localization within that first cell cycle. CckA-GFP remained bipolar (Fig. 5A, S5A), suggesting that an excess of PopZ does not disrupt recruitment or retention of CckA in these conditions. In contrast, ChpT-sfGFP completely failed to accumulate at the swarmer pole following popZ overexpression, with a significant drop in the ChpT-sfGFP cytoplasmic signal and concomitantly stronger signal at the old, stalked pole (Fig. 5B, S5B). These results indicated that ChpT is indeed excluded from the swarmer pole microdomain and that an additional component is necessary for ChpT to access that pole. Additionally, overexpressing popZ has recently been shown to reduce CtrA activation (Lasker et al., 2020), consistent with a reduction of ChpT access to the new pole where phosphotranfer from CckA to ChpT to CtrA can occur.

288 Our single-cell observations showed that (i) overexpression of wild-type parA led to prolonged localization of ChpT-sfGFP at the swarmer pole compared to wild-type cells (compare Fig. 4A and 4F) and (ii) in cells overexpressing $\operatorname{parA}(K 20 R)$, which we found prevents ChpT-sfGFP 291 localization (Fig. 4E), ParA accumulation at the swarmer pole is reduced (Laloux and Jacobs292 Wagner, 2013). Given these observations, we hypothesized that ParA may normally promote 
293 ChpT localization to the new pole in predivisional cells and provide it access to the PopZ 294 microdomain.

295 Consistent with a role for ParA in recruiting ChpT, we found that cells expressing an ectopic copy 296 of parA(K20R) also developed a single dynamic and non-polar internal cluster of CckA-GFP, DivL297 GFP, and ChpT-sfGFP (Fig. 4D-E, S4A). This internal cluster was seen in a majority of elongated 298 cells for each fusion protein (70\% for CckA-GFP, 96\% for DivL-GFP and 86\% for ChpT-sfGFP 299 with $n=50$ elongated cells in each case). These internal clusters arose relatively late in our time300 lapse experiments, likely after additional rounds of DNA replication had initiated at the stalked 301 pole, as evidenced by the fact that MipZ-CFP also formed internal clusters in elongated cells 302 overexpressing $\operatorname{parA}(K 20 R)$ (Fig. 4C). However, whereas MipZ-CFP often accumulated in 303 multiple internal clusters (Fig. 4C), we only ever saw the formation of a single internal cluster of 304 CckA-GFP, DivL-GFP, and ChpT-sfGFP (Fig. 4D-E, S4A). We hypothesized that ParA(K20R) was accumulating at one of the newly formed parS/ParB/MipZ complexes (next to a newly replicated origin) and was recruiting CckA-GFP, DivL-GFP, and ChpT-sfGFP to this cytoplasmic position within the cell. To test this hypothesis, we expressed ParA(K20R) fused to CFP in cells also expressing YFP-MipZ or ChpT-YFP. We found that ParA(K20R)-CFP accumulated in a single internal cluster that colocalized with one of the YFP-MipZ internal clusters (Fig. S5C) and with the single ChpT-YFP internal cluster (Fig. 5C).

311 Previous work found that ChpT, CckA, and ParA all interact with PopZ (Schofield et al., 2010;

312 Holmes et al., 2016). We therefore tested if PopZ was accumulating at these internal clusters and 313 recruiting CckA and ChpT. In elongated cells expressing parA(K20R), we found that while PopZ314 YFP accumulated strongly at the swarmer pole, very little PopZ-YFP signal was found in internal 315 clusters (Fig. S4C), suggesting that ChpT and CckA accumulation in the internal clusters does 316 not depend on PopZ.

\section{ParA not bound to DNA recruits ChpT to the swarmer pole}

318 Our results suggest that ChpT-sfGFP gets recruited to internal parS/ParB/MipZ complexes only 319 when an excess of ParA molecules accumulates at that complex (Fig. 4E-F, 5C). In wild-type 320 stalked cells undergoing chromosome translocation, dimers of ParA-ATP coat the nucleoid and 321 the newly formed parS/ParB/MipZ complex moves toward the swarmer pole through ParB322 mediated hydrolysis of ParA-ATP into ParA-ADP (Ptacin et al., 2010; Schofield et al., 2010; 323 Shebelut et al., 2010; Lim et al., 2014). ParA-ADP molecules released from the DNA are thought 324 to relocate to the swarmer pole where they bind PopZ (Ptacin et al., 2014) (Fig. 3A). We 325 hypothesized that ChpT is normally targeted to the swarmer pole or to internal clusters by binding 
ParA molecules not bound to DNA. Consistent with this hypothesis, we did not observe ChpT moving across cells with the translocating chromosome in wild-type cells when ParA is mostly nucleoid-associated (Fig. 4A). To further test this hypothesis and to examine whether ParA and ChpT interact, we used a bacterial two-hybrid system in which two proteins of interest are fused to the T18 and T25 portions of adenylate cyclase. If the two proteins interact, they reconstitute adenylate cyclase activity, leading to production of a red pigment. We did not detect any interaction between ChpT and wild-type ParA (Fig. 5D). However, when testing ChpT and ParA(R195E), a DNA-binding deficient mutant of ParA (Ptacin et al., 2010; Schofield et al., 2010; Lim et al., 2014), we now observed colonies with intense red staining, indicative of an interaction (Fig. 5D), and supporting a model in which ChpT can bind ParA that is not bound to DNA.

336 ParA can dimerize and cycle through both ATP and ADP bound states. To test how these features of ParA impact interaction with ChpT, we tested different point mutants of ParA using our twohybrid assay: ParA(G16V), a dimerization-deficient mutant that can presumably bind ATP or ADP but not DNA; ParA(K20R), an ATP-binding deficient mutant described above; and ParA(D44A), an ATP-locked mutant (Ptacin et al., 2010). For G16V, we still observed some red staining, suggesting that this variant still binds ChpT (Fig. S5D), likely because disrupting dimerization prevents or diminishes DNA binding by ParA. For K20R and D44A, these mutants of ParA did not interact with ChpT (Fig. S5D). However, combining each with the R195E mutation that disrupts DNA-binding produced red colonies indicative of an interaction (Fig. S5D). We conclude that the DNA-binding status of ParA, and not its ATP/ADP state, is most critical for an interaction with ChpT.

347 We also detected an interaction between the histidine kinase domain of CckA (CckA $\mathrm{HK}_{\text {) }}$ and $348 \operatorname{ParA}(\mathrm{R} 195 \mathrm{E})$, but not with wild-type ParA (Fig. 5D). Further, we found that ChpT and CckA $\mathrm{HK}_{\mathrm{H}}$ can each interact to some extent with ParB in our bacterial two-hybrid assay (Fig. 5D), but did not observe any interaction with either MipZ or the DNA-binding deficient mutant MipZ(R194A) (Corrales-Guerrero et al., 2020) (Fig. 5D). Finally, we found that the C-terminal domain of ChpT, which weakly resembles the ATP-binding domain of a histidine kinase (Fioravanti et al., 2012; Blair et al., 2013), was required for its interaction with ParA and ParB (Fig. 5D). The N-terminal domain of ChpT, which resembles the $\mathrm{DHp}$ domain found in histidine kinases, is critical for mediating phosphotransfer from CckA and to CtrA. The function of ChpT's C-terminal domain had been unclear, although required for CckA binding (Fig. 5D), but our results now suggest it mediates binding to ParA that is not bound to the nucleoid. 
Based on our bacterial two-hybrid assay, we infer that ChpT and CckA are recruited to internal clusters by excess levels of ParA that are unbound to DNA and associated with a ParB/parS complex. However, while ChpT recruitment to the new pole is disrupted when chromosome segregation is incomplete, CckA polarization is not (Iniesta et al., 2010b), likely because it depends on PodJ (Fig. 1D-E) rather than ParA (Fig. 4B, D). To confirm that ChpT was being recruited to internal clusters independent of CckA, we constructed, informed by the available ChpT structures (Fioravanti et al., 2012; Blair et al., 2013), a ChpT variant containing the substitutions R167E, R169E, and R171E, which we call ChpT*, that cannot bind CckA but retains interaction with ParA(R195E) and ParB (Fig. 5D). We then ectopically expressed either ChpT or $\mathrm{ChpT}^{*}$ fused to sfGFP in cells with a vanillate-inducible copy of $\operatorname{parA}(K 20 R)$. In the absence of vanillate, ChpT-sfGFP localized to both poles (Fig. S5E), but ChpT*-sfGFP did not localize at either cell pole at any stage of the cell cycle (Fig. S5F). These results support the notion that ChpT interaction with CckA is necessary but not sufficient for ChpT to accumulate at the poles. In the presence of vanillate to induce parA(K20R), ChpT-sfGFP accumulated at the old, stalked pole and in an internal cluster (Fig. 5E). By contrast, ChpT*-sfGFP accumulated only in an internal cluster (Fig. 5F). These results demonstrate that ChpT recruitment to internal clusters occurs in a CckA-independent manner and likely reflects its direct interaction with the chromosome segregation machinery. Taken all together, our results support a model in which chromosome translocation normally leads to an accumulation of ParA unbound to DNA at the nascent swarmer pole, which recruits ChpT to enable phosphotransfer from CckA to CtrA and, ultimately, successful completion of the cell cycle.

\section{Discussion}

\section{A substrate-product relationship that couples cell division to DNA replication}

381 How organisms ensure the correct order of events during the cell cycle is critical to their survival. Hartwell and Weinert outlined two general mechanisms for enforcing order (Hartwell and Weinert, 1989). One involves dedicated checkpoints, which they envisioned as surveillance systems that are not involved in executing either of two events but are involved only in monitoring and promoting their relative order of execution. The second involves substrate-product relationships in which the product of one event is the substrate for the next, thereby intrinsically ensuring they occur only in succession. Here, we uncovered a substrate-product relationship that couples cell division in Caulobacter to the successful initiation of DNA replication (Fig. 6). The late stages of the Caulobacter cell cycle include flagellar and pili biogenesis, as well as cell division. These 
localization of these latter two factors to the swarmer cell pole is necessary for CtrA activation and dependent on the initiation of DNA replication (Fig. 1D-G, Fig. 6) (Iniesta et al., 2010b). DnaA, likely in the ATP-bound form, is a transcription factor that promotes the synthesis of PodJ (Hottes et al., 2005), which then recruits CckA and DivL to the swarmer pole. However, even in cells ectopically producing PodJ (and, consequently, with CckA and DivL at the pole), CtrA is not activated (Fig. 1B-C, Fig. 6). We found that ChpT is not localized unless the ori-proximal region of a newly replicated chromosome is properly translocated across the cell to the swarmer pole (Fig. 4E, Fig. 6). Our findings suggest that ParA, which is nucleoid-associated when promoting the directional movement of the parS/ParB complex, eventually accumulates at the pole and is not bound to DNA, enabling it to recruit ChpT and complete the CckA-ChpT-CtrA phosphorelay (Fig. 6). In this manner, the translocating chromosome is a physical cue that ultimately triggers CtrA activation, thereby ensuring that cell division, and other CtrA-dependent processes, do not occur until after DNA replication has successfully initiated.

404 The use of chromosome position to couple cell-cycle processes also arises in many species to tie the final stages of chromosome segregation to the onset of cytokinesis. For instance, in E. coli, unsegregated chromosomes at mid-cell accumulate the cell division inhibitor SImA such that cytokinesis cannot occur until after chromosomes are properly separated away from mid-cell (Bernhardt and De Boer, 2005). Noc in B. subtilis (Wu and Errington, 2004) and MipZ in C. crescentus (Thanbichler and Shapiro, 2006) play analogous roles. Notably, these systems for coupling chromosome segregation to cell division and the mechanism identified here for coupling

411 replication to cell division feature substrate-product relationships, rather than checkpoint 412 mechanisms involving regulatory feedback systems, as is commonly found in eukaryotes. 413 Understanding why bacteria may rely more heavily on substrate-product relationships to order 414 their cell cycles remains a fascinating question for the future.

\section{Activation of a signaling pathway by the physical translocation of a chromosome}

416 For most two-component signaling pathways, the signals that activate them remain unknown. For 417 cases in which a signal has been identified, most feature small molecules or low molecular weight 418 proteins that bind to a periplasmic or transmembrane domain of a top-level histidine kinase. 419 Although CckA and its activator DivL both reside in the inner membrane, no periplasmic or 420 extracellular signals have been identified that regulate their activities. DivL is directly regulated by 421 the cytoplasmic response regulator DivK (Tsokos et al., 2011) and CckA by cytoplasmic c-di-GMP 422 (Lori et al., 2015). Our work here now indicates that a major cue for the CtrA phosphorelay 423 involves the initially duplicated region of the chromosome, which serves to recruit ChpT via the 
424 ParA and ParB segregation complex. There is some precedent for signal sensing by intermediate 425 components of complex phosphorelays, with Rap phosphatases in B. subtilis known to regulate 426 the response regulator Spo0B, which shuttles phosphoryl groups from KinA/B/C/D/E to the 427 histidine phosphotransferase SpoOF (Solomon et al., 1996; Perego, 2013). However, in that 428 system, the Rap phosphatases respond to small Phr peptides, not a physical cue like 429 chromosome translocation.

430 Notably, our work indicates that CckA alone is not sufficient to recruit ChpT to the swarmer pole, 431 despite being sufficient to bind and phosphorylate ChpT in vitro (Biondi et al., 2006). This 432 difference likely reflects a constraint, or barrier, imposed by the PopZ matrix that is established at 433 the swarmer cell pole. PopZ forms a dense, interconnected matrix with possible phase-separated 434 properties that enable the compartmentalization of the cytoplasm (Bowman et al., 2010; Schofield 435 et al., 2010). We found that ChpT cannot access this polar microdomain unless translocation of 436 the parS/ParB complex has occurred, likely because the movement of this complex across the 437 cell is required to produce a pool of ParA at the pole that is not nucleoid bound. Although CckA is 438 not sufficient to recruit ChpT inside the PopZ matrix, ChpT interaction with CckA is required for its accumulation at the nascent swarmer pole.

440 We found that in a bacterial two-hybrid system, ChpT and CckA could interact with ParA, but only 441 if ParA was not DNA bound. Additionally, we found that in Caulobacter cells in which chromosome 442 translocation was disrupted, ChpT and CckA were recruited to internal sites within the cytoplasm 443 harboring the parS/ParB/ParA machinery. However, only ChpT access to the swarmer pole was 444 dependent on a successful segregation of the chromosome; CckA accumulation at the swarmer 445 pole was not. Importantly, ChpT co-localization with the Par machinery at internal clusters 446 occurred even for a ChpT variant engineered to ablate binding to CckA, supporting the notion that 447 ChpT recruitment by ParA and ParB is independent of CckA. Whether ChpT binds simultaneously 448 to both ParA/ParB and CckA/CtrA at the swarmer pole is not yet clear. Further work is needed to 449 explore the biochemical and biophysical details of ChpT's multiple protein-protein interactions.

\section{Concluding Remarks}

451 Our results indicate that the PopZ microdomain may serve multiple functions in Caulobacter. As 452 suggested previously, it may increase the effective concentration of various signaling proteins at 453 the pole to promote or increase their activities (Bowman et al., 2010; Holmes et al., 2016; Lasker 454 et al., 2020). In addition, we propose that it also serves as a gatekeeper that selectively excludes 455 some proteins like ChpT until others have arrived, thereby providing regulatory capabilities to 456 cells. In a similar way, phase-separated granules and bodies in the cytoplasm of eukaryotic cells 
457 are emerging as powerful regulators and organizers of many cellular processes (Banani et al.,

458 2017; Gomes and Shorter, 2019).

459 The mechanism identified here in which chromosome translocation serves as a physical cue to

460 trigger a signaling pathway is likely conserved. Homologs of ChpT, PopZ, and the parS/ParB/ParA

461 system are found throughout the a-proteobacteria and could work in similar ways. The Par system

462 is found even more broadly and it has become increasingly clear that ParB and ParA can interact

463 with a wide variety of proteins other than each other (Thanbichler and Shapiro, 2006; Gruber and

464 Errington, 2009; Schofield et al., 2010; Mercy et al., 2019; Kawalek et al., 2020; Pióro and

465 Jakimowicz, 2020), and so could participate in the regulated recruitment of proteins in other

466 contexts and for other purposes.

467 In sum, our work reveals a new mechanism by which bacterial cells coordinate two cell-cycle 468 events, ensuring that cell division does not inadvertently occur before DNA replication in $C$. 469 crescentus. Although the timing and execution of each individual process - DNA replication 470 initiation and cell division - has been extremely well-studied, how cells sense whether the former 471 has occurred and relay this information to the latter has remained unclear. We found a novel 472 connection linking the physical translocation of the origin-proximal region of a chromosome to the 473 activation of a signaling pathway that controls cell-cycle progression. Chromosome movement 474 and positioning is a common signal in eukaryotes; for instance, the failure to properly segregate 475 chromosomes activates the spindle checkpoint pathway (Gorbsky, 2015). Our work suggests that 476 bacteria may similarly use chromosome dynamics as an important cue for regulating cell-cycle 477 progression. 


\section{Acknowledgments}

480 We thank S. Srikant, K. Gozzi, M. Guo, and C. Tsokos for comments on the manuscript, and C.

481 Tsokos and J. Dyer for early discussions on the project. Instrumentation resources from the 482 BioMicro Center in the Department of Biology at MIT are gratefully acknowledged. This work was 483 supported by a long-term fellowship (LT000322/2017-L) from the Human Frontier Science 484 Program to M.G. and an NIH grant to M.T.L. (R01GM082899), who is also an Investigator of the 485 Howard Hughes Medical Institute.

\section{Author Contributions}

487 M.G. performed all experiments, with assistance from A.G.S and L.K.C. M.G. and M.T.L. designed 488 experiments, analyzed data, prepared figures, and wrote the manuscript.

\section{Declaration of Interests}

490 The authors declare no competing interests. 


\section{Figure Legends}

493 Figure 1: Polarization of CckA and DivL is not sufficient to activate CtrA if DNA replication 494 is inhibited.

495 (A) Schematic of the Caulobacter crescentus cell cycle.

496 (B) CtrA immunoblots in synchronized cells expressing $d n a A$ (+IPTG) or depleted of $d n a A(-I P T G)$ 497 with ectopic expression of ctrA (+0.075\% xyl) and podJ (+cumate) when indicated. Times indicate 498 minutes post-synchronization. Graph shows CtrA band intensity normalized to RpoA control.

499 (C) mRNA levels of the CtrA-activated gene divK measured by qRT-PCR and normalized to $r p o A$ 500 mRNA levels in cells expressing dnaA (+IPTG) or depleted of dnaA (-IPTG) with ectopic 501 expression of the proteolytically stable mutant $\operatorname{ctr} A \Delta 3 \Omega(+0.075 \% \mathrm{xyl})$ and podJ (+cumate) when 502 indicated.

503 (D-G) Localization of CckA-GFP (E), DivL-GFP (F) and ChpT-YFP (G) in fixed predivisional cells 504 (90 min. post-synchronization) expressing dnaA (+IPTG) or depleted of dnaA (-IPTG) with or 505 without ectopic expression of podJ (+cumate). Green arrows indicate the swarmer pole of one 506 representative cell for each condition. (D) Histogram represents the percentage of cells with 507 detectable foci of CckA-GFP, DivL-GFP, or ChpT-YFP at the swarmer pole (includes bipolar and 508 unipolar clusters at the swarmer pole) in each condition. Total number of cells examined from two 509 biological replicates is indicated in each case. Scale bar $=2 \mu \mathrm{m}$.

510 Figure 2: Replication of the full chromosome is not required for CtrA activation.

511 (A) qPCR on genomic DNA extracted from cells expressing $d n a A$ (+IPTG) or depleted of $d n a A$ 512 and expressing the $\operatorname{dnaA}(R 357 A)$ ATP-locked mutant (-IPTG +xyl) at different timepoints post513 synchronization. Map of $C$. crescentus chromosome (right) shows the localization of primers used 514 to assess replication and their distances from oriC. Samples were normalized internally to DNA 515 levels close to the terminus (CCNA_01869) and each timepoint was then normalized to $\mathrm{t}=0 \mathrm{~min}$. 516 to evaluate fold-change from $1 \mathrm{~N}$. Note that for the ' $+d n a A$ ' condition, samples after $\mathrm{t}=90 \mathrm{~min}$. were 517 excluded because DNA close to the terminus has been replicated.

518 (B) CtrA protein levels at the times indicated post-synchronization in cells expressing $d n a A$ 519 (+IPTG) or depleted of $\operatorname{dnaA}(-\mathrm{IPTG})$ with ectopic expression of wild-type ctrA (+van) and with $520(+x y l)$ or without ectopic expression of $d n a A(R 357 A)$. Graph shows CtrA protein band intensity 521 normalized to RpoA. 
522 (C) mRNA levels of the CtrA-activated genes divK and $p d e A$ measured by qRT-PCR and

523 normalized to rpoA mRNA levels for cells treated as in (B).

524 Figure 3: CtrA activation in predivisional cells is reduced when chromosome segregation

525 is perturbed.

526 (A) Schematic of chromosome segregation during the C. crescentus cell cycle.

527 (B) mRNA levels of the CtrA-activated genes divK and $p d e A$ measured by qRT-PCR and 528 normalized to $r p o A$ mRNA levels at the times indicated post-synchronization in cells expressing 529 (+van) or not (-van) parA(K20R). Expression of parA(K20R) was induced 60 min. pre530 synchronization and post-synchronization with $500 \mu \mathrm{M}$ vanillate. Data represent the mean \pm SD of 531 three biological replicates.

532 (C) CtrA protein levels at the times indicated post-synchronization in cells from the same 533 conditions as in (B). Graph shows CtrA protein band intensity normalized to RpoA.

534 (D) mRNA levels of the CtrA-activated genes divK and $p d e A$ measured by qRT-PCR and 535 normalized to rpoA mRNA levels at the times indicated post-synchronization in cells expressing 536 (+van) or not (-van) the spreading-deficient parB(G101S) mutant. Expression of parB(G101S) 537 was induced $60 \mathrm{~min}$. pre-synchronization and post-synchronization with $500 \mu \mathrm{M}$ vanillate. Data 538 represent the mean \pm SD of three biological replicates.

539 (E) CtrA protein levels at the times indicated post-synchronization in cells from the same 540 conditions as in (D). Graph shows CtrA protein band intensity normalized to RpoA.

541 (F) Relative fold-change in mRNA levels of the CtrA-regulated gene divK measured by qRT-PCR 542 and normalized to $r p o A$ mRNA levels at timepoint 100 min. post-synchronization, when inducing 543 parB or $\operatorname{parB}(G 101 S) 45 \mathrm{~min}$., $60 \mathrm{~min}$. or $90 \mathrm{~min}$. before synchronization compared to the 544 uninduced condition. Shown are averages from two biological replicates with the individual 545 datapoints for each replicate.

546 Figure 4: ChpT access to the new swarmer pole is blocked when chromosome segregation 547 is disrupted.

548 (A) Time lapse of ChpT-sfGFP dynamics in a wild-type cell, imaged at 10 min. intervals from 60 549 to $150 \mathrm{~min}$. (before division) post-synchronization, with consecutive frames pasted side-by-side 550 to generate the concatenated figure. Green arrow indicates ChpT-sfGFP transient localization at 551 the new pole. Scale bar $=1 \mu \mathrm{m}$. 
552 (B) Percentage of cells with detectable foci at the swarmer pole at any time during time-lapse 553 imaging of MipZ-CFP, CckA-GFP, DivL-GFP, and ChpT-sfGFP without parA(K20R) induction 554 (dividing cells; -van) and with parA(K20R) induction (elongated cells only; +van). Expression of $555 \operatorname{parA}(K 20 R)$ was induced $30 \mathrm{~min}$. before synchronization and after synchronization with $500 \mu \mathrm{M}$ 556 vanillate. Total number of cells examined from two biological replicates is indicated in each case.

557 (C-E) Examples of a single elongated cell from each condition quantified in (B). Scale bars = 1 $558 \mu \mathrm{m}$. (C) Time-lapse of MipZ-CFP dynamics within a single cell expressing the dominant negative $559 \operatorname{parA}(K 20 R)$ mutant, imaged at $10 \mathrm{~min}$. intervals from 50 to $270 \mathrm{~min}$. post-synchronization, with 560 consecutive timeframes pasted side-by-side to generate the concatenated figure (left) and corresponding kymograph (right). Red arrows indicate MipZ-CFP internal clusters next to the newly replicated chromosomal origins. (D) Same as (C) but for CckA-GFP. Green arrow shows CckA-GFP localization at the new swarmer pole upon expression of parA(K20R). Red arrow shows CckA-GFP accumulation in a single internal cluster. (E) Same as (C) but for ChpT-sfGFP. Red arrow shows ChpT-sfGFP accumulation in a single internal cluster.

(F) Timelapse of ChpT-sfGFP dynamics within a single cell expressing ectopic wild-type parA, imaged at $10 \mathrm{~min}$. intervals from 50 to $270 \mathrm{~min}$. post-synchronization, with consecutive timeframes pasted side-by-side to generate the concatenated figure (left) and corresponding kymograph (right). Expression of parA was induced $30 \mathrm{~min}$. before synchronization and during imaging postsynchronization in the agarose pad with addition of $500 \mu \mathrm{M}$ vanillate. Green arrow shows ChpTsfGFP localization at the new swarmer pole upon expression of parA. Red arrow shows ChpTsfGFP accumulation in a single internal cluster. Scale bar $=1 \mu \mathrm{m}$.

(G) Percentage of cells with detectable foci at the swarmer pole at any time during time lapse imaging of ChpT-sfGFP (see Fig. 4F) and MipZ-CFP (see Fig. S4B) ( $n=100$ cells for each condition) without (-van condition) or with parA induction (+van).

Figure 5: ParA not bound to DNA recruits ChpT to the swarmer pole

577 (A-B) Fluorescence micrographs (top) of CckA-GFP (A) or ChpT-sfGFP (B) in cells expressing $\operatorname{parA}(K 20 R)(+\mathrm{van})$ with (+xyl) or without (-xyl) induction of popZ post-synchronization (for full time lapses see Fig. S5A-B for CckA-GFP and ChpT-sfGFP, respectively). Graphs (bottom) show the average fluorescence intensities (solid line) from the old pole to the new pole from 15 cells for each condition. Shading around the solid line represents the SD. Scale bars $=1 \mu \mathrm{m}$.

582 (C) ChpT-YFP internal cluster colocalizes with ectopically produced ParA(K20R)-CFP. Time583 lapse of ChpT-YFP and ParA(K20R)-CFP dynamics within a single cell expressing parA(K20R) 
584 (+van), imaged at $10 \mathrm{~min}$. intervals from 150 to $330 \mathrm{~min}$. post-synchronization, with consecutive

585 frames pasted side-by-side to generate the concatenated figure (left) and corresponding 586 kymograph (right). parA(K20R) was induced $30 \mathrm{~min}$. pre-synchronization and during imaging 587 post-synchronization in the agarose pad by the addition of $500 \mu \mathrm{M}$ vanillate. In the overlay, ChpT588 YFP is shown in green and ParA(K20R)-CFP in red. Red arrows show the ChpT-YFP and 589 ParA(K20R)-CFP internal cluster. Scale bar $=1 \mu \mathrm{m}$.

590 (D) Bacterial two-hybrid assay. BTH101 reporter cells producing the indicated proteins or domains 591 fused to the T18 or T25 domain of Bordetella adenylate cyclase were spotted on MacConkey agar 592 plates supplemented with IPTG and maltose. Interaction between two fusion proteins results in a 593 red color of the bacterial colony. ChpT $\mathrm{DHp}_{\mathrm{D}}$ : ChpT Dimerization and Histidine phosphotransfer 594 domain; CckA $A_{H K}$ : CckA Histidine Kinase domain; CckARD: CckA receiver domain; ChpT*: 595 ChpT(R167E, R169E, R171E).

596 (E) Time-lapse imaging of ChpT-sfGFP dynamics when produced (+xyl) in cells expressing parA(K20R) (+van), imaged at 10 min. intervals from 30 to 270 min. post-synchronization, with consecutive frames pasted side-by-side to generate the concatenated figure. $\operatorname{par} A(K 20 R)$ was induced $30 \mathrm{~min}$. pre-synchronization and during imaging post-synchronization in the agarose pad with addition of $500 \mu \mathrm{M}$ vanillate. chpT-sfGFP was induced at $\mathrm{t}=0 \mathrm{~min}$. after synchronization and in the agarose pad with $0.3 \%$ xylose. Red arrow shows the ChpT-sfGFP internal cluster. Scale bar $=1 \mu \mathrm{m}$.

(F) As in (E) but for ChpT(R167E,R169E,R171E)-sfGFP (ChpT*-sfGFP). Note that ChpT*-sfGFP still forms a single internal cluster (red arrow). Scale bar $=1 \mu \mathrm{m}$.

Figure 6: Model for coupling DNA replication initiation and proper chromosome translocation to the activation of the CckA-ChpT signaling pathway

607 Schematic of the polarization of CtrA regulators at the new pole. In wild-type conditions (top 608 panel), initiation of DNA replication and proper accumulation of ParA at the new pole within the 609 PopZ matrix enables accumulation of the ChpT intermediate component of the CckA-ChpT-CtrA 610 phosphorelay. In cells that do not initiate DNA replication (bottom panel), production of the 611 scaffold protein PodJ can restore the polarization of CckA and DivL, but does not allow ChpT 612 accumulation within the PopZ matrix, leading to an incomplete pathway missing the intermediate 613 ChpT histidine phosphotransferase for CtrA activation. 


\section{Supplemental Figure Legends}

616 Figure S1: Polarization of CckA and DivL is not sufficient to activate CtrA in predivisional 617 cells in the absence of DNA replication.

618 (A) Schematic of the CckA-ChpT phosphorelay activity at the new pole in predivisional cells.

619 (B) Flow cytometry profiles after SYTOX staining showing DNA content of synchronized cells 620 expressing $d n a A(+\mathrm{IPTG})$ or depleted of $d n a A(-\mathrm{IPTG})$ with ectopic expression of $\operatorname{ctr} A(+0.075 \%$ $621 \mathrm{xyl}$ ) and podJ (+cumate) when indicated.

622 (C) mRNA levels of the ctrA and podJ genes measured by qRT-PCR and normalized to rpoA 623 mRNA levels in cells expressing dnaA (+IPTG) or depleted for dnaA (-IPTG) with ectopic 624 expression of the stable ctrA point mutant $\operatorname{ctrA} \triangle 3 \Omega(+0.075 \% \mathrm{xyl})$ and podJ (+cumate) when 625 indicated.

626 (D) $C \operatorname{tr} A \Delta 3 \Omega$ protein levels in cells depleted for $d n a A$ (-IPTG) with ectopic expression of the stable 627 ctrA point mutant $\operatorname{ctr} A \Delta 3 \Omega(+0.075 \% \mathrm{xyl})$ when indicated.

628 (E) CtrA and GcrA protein levels at the times indicated post-synchronization in cells expressing $629 d n a A(+I P T G)$ or depleted of $d n a A$ (-IPTG) with ectopic expression of wild-type ctrA (+van) and 630 gcrA-3xflag (+xyl) when indicated. Graphs show CtrA and GcrA protein band intensity normalized 631 to RpoA.

632 (F) mRNA levels of the CtrA-activated gene divK measured by qRT-PCR and normalized to rpoA 633 mRNA levels at the times indicated post-synchronization in cells expressing dnaA (+IPTG) or 634 depleted of $d n a A$ (-IPTG) with ectopic expression of the proteolytically stable mutant $\operatorname{ctr} A \Delta 3 \Omega$ 635 (+van) and with (+xyl) or without ectopic expression of gcrA-3xflag.

636 (G) CtrA protein levels in cells expressing (+ $50 \mu \mathrm{M}$ van) or not (-van) the PA5295 637 phosphodiesterase in cells depleted for dnaA (-IPTG).

$638(\mathrm{H})$ mRNA levels of the CtrA-activated gene divK measured by qRT-PCR and normalized to $r p o A$ 639 mRNA levels at the times indicated post-synchronization in cells expressing dnaA (+IPTG) or 640 depleted of $d n a A$ (-IPTG) and the Pseudomonas aeruginosa phosphodiesterase PA5295 (+50 $641 \mu \mathrm{M}$ van) when indicated. This strain also expressed an ectopic copy of podJ (+cumate), when 642 indicated. 
643 (I) SciP protein levels at the times indicated post-synchronization in cells expressing $d n a A$

644 (+IPTG) or depleted of $\operatorname{dnaA}(-\mathrm{IPTG})$ with ectopic expression of ctrA (+xyl) and podJ (+cumate)

645 when indicated.

646 Figure S2: Replication of the full chromosome is not required for CtrA activation.

647 (A) Flow cytometry profiles after SYTOX staining showing DNA content of synchronized cells 648 expressing $d n a A(+\mathrm{IPTG})$ or depleted of $d n a A(-\mathrm{IPTG})$ with ectopic expression of ctrA (+van) and $649 d n a A(R 357 A)(+x y l)$ when indicated.

650 (B) ctrA mRNA levels measured by qRT-PCR and normalized to rpoA mRNA levels in cells 651 expressing dnaA (+IPTG) or depleted of $d n a A$ (-IPTG) with ectopic expression of $d n a A(R 357 A)$ $652(+x y l)$ when indicated.

653 (C) GcrA protein levels in cells expressing dnaA (+IPTG) or depleted of dnaA (-IPTG) with ectopic 654 expression of wild-type ctrA (+van) and $d n a A(R 357 A)(+x y l)$. RpoA is shown as a loading control.

655 (D) mRNA levels of ctrA measured by qRT-PCR and normalized to rpoA mRNA levels in cells 656 expressing dnaA (+IPTG) or depleted of $d n a A$ (-IPTG) with ectopic expression of ctrA (+van) and $657 d n a A(R 357 A)(+x y l)$ when indicated.

658 Figure S3: CtrA activation in predivisional cells is reduced when chromosome segregation 659 is perturbed.

660 (A) Flow cytometry profiles after SYTOX staining showing DNA content of synchronized cells with 661 (+van) or without (-van) ectopic expression of parA(K20R). Expression of parA(K20R) was 662 induced $60 \mathrm{~min}$. before synchronization and after synchronization with $500 \mu \mathrm{M}$ vanillate.

663 (B) Flow cytometry profiles after SYTOX staining showing DNA content of synchronized cells with 664 (+van) or without (-van) ectopic expression of parB(G101S). Expression of parB(G101S) was 665 induced $60 \mathrm{~min}$. before synchronization and after synchronization with $500 \mu \mathrm{M}$ vanillate.

666 (C) Time lapse of MipZ-CFP dynamics within a single cell expressing the parB(G101S) mutant, 667 imaged at $10 \mathrm{~min}$. intervals from 50 to $270 \mathrm{~min}$. post-synchronization, with consecutive frames 668 pasted side-by-side to generate the concatenated figure (left) and corresponding kymograph 669 (right). Expression of parB(G101S) was induced $30 \mathrm{~min}$. before synchronization and during 670 imaging post-synchronization in the agarose pad with addition of $500 \mu \mathrm{M}$ vanillate. The red arrows 671 indicate MipZ-CFP internal clusters next to the newly replicated chromosomal origins. Scale bar $672=1 \mu \mathrm{m}$. 
673 Figure S4: ChpT access to the new swarmer pole is affected when chromosome 674 segregation is disrupted.

675 (A) Time lapse of DivL-GFP dynamics within a single cell expressing the dominant negative $676 \operatorname{parA}(K 20 R)$ mutant, imaged at $10 \mathrm{~min}$. intervals from 50 to $270 \mathrm{~min}$. post-synchronization, with 677 consecutive frames pasted side-by-side to generate the concatenated figure (left) and 678 corresponding kymograph (right). Expression of parA(K20R) was induced 30 min. before 679 synchronization and during imaging post-synchronization in the agarose pad with addition of 500 $680 \mu \mathrm{M}$ vanillate. The green arrow shows DivL-GFP localization at the new swarmer pole upon 681 expression of parA(K20R). The red arrow shows DivL-GFP accumulation in a single internal 682 cluster. Scale bar $=1 \mu \mathrm{m}$.

683 (B) Timelapse of MipZ-CFP dynamics within a single cell expressing ectopic wild-type parA, 684 imaged at $10 \mathrm{~min}$. intervals from 20 to $260 \mathrm{~min}$. post-synchronization, with consecutive timeframes 685 pasted side-by-side to generate the concatenated figure (left) and corresponding kymograph 686 (right). Expression of parA was induced $30 \mathrm{~min}$. before synchronization and during imaging post687 synchronization in the agarose pad with addition of $500 \mu \mathrm{M}$ vanillate. Green arrow shows MipZ688 CFP localization at the new swarmer pole upon expression of parA. Red arrow shows MipZ-CFP 689 internal cluster. Scale bar $=1 \mu \mathrm{m}$.

690 (C) As in (A) but for PopZ-YFP dynamics. The green arrow shows PopZ-YFP localization at the 691 new swarmer pole upon expression of $\operatorname{parA}(K 20 R)$. Scale bar $=1 \mu \mathrm{m}$.

\section{Figure S5: ParA not bound to DNA recruits ChpT to the swarmer pole}

693 (A-B) Full time lapses of CckA-GFP (A) and ChpT-sfGFP (B) dynamics in cells expressing parA(K20R) (+van) with (+xyl) or without (-xyl) induction of popZ expression, corresponding to Fig. $5 \mathrm{~A}$ and $\mathrm{B}$ respectively. Scale bar $=1 \mu \mathrm{m}$.

(C) YFP-MipZ internal clusters colocalize with ectopically produced ParA(K20R)-CFP. Time lapse imaging of YFP-MipZ and ParA(K20R)-CFP dynamics within a single cell expressing parA(K20R)

698 (+van), imaged at $10 \mathrm{~min}$. intervals from 150 to $330 \mathrm{~min}$. post-synchronization, with consecutive 699 frames pasted side-by-side to generate the concatenated figure (left) and corresponding 700 kymograph (right). Expression of parA(K20R) was induced $30 \mathrm{~min}$. before synchronization and 701 during imaging post-synchronization in the agarose pad with addition of $500 \mu \mathrm{M}$ vanillate. In the 702 overlay, YFP-MipZ is shown in green and ParA(K20R)-CFP in red. Red arrows show the YFP703 MipZ and ParA(K20R)-CFP internal cluster. Scale bar $=1 \mu \mathrm{m}$. 
704 (D) Bacterial two-hybrid assay testing the interaction of ChpT fused to the T25 Bordetella

705 adenylate cyclase domain with ParA and ParA point mutants fused to the Bordetella T18

706 adenylate cyclase domain. When spotted on MacConkey agar plates supplemented with IPTG

707 and maltose, interactions between the two fusion proteins result in a red color shift of the bacterial

708 colony.

709 (E-F) Time lapse of the dynamics of ectopic ChpT-sfGFP and the ChpT(R167E,R169E,R171E)-

710 sfGFP (ChpT*-sfGFP) mutant (+xyl) in cells that are not expressing parA(K20R). Compare to Fig.

$7115 \mathrm{E}$ and F, respectively. Note that the $\mathrm{ChpT}^{*}$-sfGFP point mutant fails to accumulate at the new

712 pole prior to cell division, compared to ectopically produced wild-type ChpT-sfGFP (red arrow).

713 Scale bar $=1 \mu \mathrm{m}$. 


\section{Methods}

\section{Growth conditions}

717 Caulobacter crescentus strains were grown in PYE (2 g/L bactopeptone, $1 \mathrm{~g} / \mathrm{L}$ yeast extract, 0.3 $\left.718 \mathrm{~g} / \mathrm{L} \mathrm{MgSO}_{4}, 0.5 \mathrm{mM} \mathrm{0.5M} \mathrm{CaCl}_{2}\right)$ or in fresh M2G+ $\left(0.87 \mathrm{~g} / \mathrm{L} \mathrm{Na}_{2} \mathrm{HPO}_{4}, 0.53 \mathrm{~g} / \mathrm{L} \mathrm{KH}_{2} \mathrm{PO}_{4}, 0.5 \mathrm{~g} / \mathrm{L}\right.$ $719 \mathrm{NH}_{4} \mathrm{Cl}, 0.5 \mathrm{mM} \mathrm{MgSO}_{4}, 10 \mu \mathrm{M} \mathrm{FeSO}_{4}, 0.1 \mathrm{mM} \mathrm{CaCl}_{2}, 1 \% \mathrm{PYE}, 0.2 \%$ glucose) at $30^{\circ} \mathrm{C}$. Expression 720 from the $\mathrm{P}_{\text {lac }}$ promoter was induced with $1 \mathrm{mM}$ IPTG. Expression from the $\mathrm{P}_{x y l}$ promoter was 721 repressed with glucose $(0.2 \%)$ and induced with xylose $(0.3 \%)$ unless noted. Expression from the $722 \mathrm{P}_{\text {cumate }}$ promoter was induced with $0.2 \mu \mathrm{M}$ cumate. Expression from the $\mathrm{P}_{\text {van }}$ promoter was induced 723 with vanillate $(500 \mu \mathrm{M})$ unless noted. To maintain plasmids, antibiotics were added at the following 724 concentrations (liquid/plate): kanamycin $\left(5 \mu \mathrm{g} \mathrm{mL}^{-1} / 25 \mu \mathrm{g} \mathrm{mL}{ }^{-1}\right)$, oxytetracycline $(1 \mu \mathrm{g} \mathrm{mL}-1 / 2$ $\left.725 \mu \mathrm{g} \mathrm{mL}{ }^{-1}\right)$, gentamycin $\left(2.5 \mu \mathrm{g} \mathrm{mL}^{-1} / 5 \mu \mathrm{g} \mathrm{mL}^{-1}\right)$, chloramphenicol $\left(2 \mu \mathrm{gL}^{-1} / 1 \mu \mathrm{g} \mathrm{mL}^{-1}\right)$.

726 E. coli strains were grown in LB $(10 \mathrm{~g} / \mathrm{L} \mathrm{NaCl}, 10 \mathrm{~g} / \mathrm{L}$ tryptone, $5 \mathrm{~g} / \mathrm{L}$ yeast extract) and 727 supplemented with antibiotics at the following concentrations unless noted (liquid/plate): 728 kanamycin $\left(30 \mu \mathrm{g} \mathrm{mL}^{-1} / 50 \mu \mathrm{gL}^{-1}\right)$, oxytetracycline $\left(12 \mu \mathrm{g} \mathrm{mL} \mathrm{m}^{-1} / 12 \mu \mathrm{gL}^{-1}\right)$, gentamycin (15 $\left.729 \mu \mathrm{g} \mathrm{mL}-1 / 20 \mu \mathrm{g} \mathrm{mL}^{-1}\right)$, chloramphenicol $\left(20 \mu \mathrm{g} \mathrm{mL}^{-1} / 30 \mu \mathrm{g} \mathrm{mL}^{-1}\right)$, carbenicillin $\left(50 \mu \mathrm{gL} \mathrm{mL}^{-1} / 100\right.$ $\left.730 \mu \mathrm{g} \mathrm{mL}^{-1}\right)$.

\section{Strain construction}

732 All Caulobacter strains were derivative of the wild-type isolate ML76 from the CB15N/NA1000 733 strain.

734 Strains ML3364 and ML3365 were constructed using ФCr30 phage transduction (Ely, 1991) to 735 move pMT585-gcrA-3xFLAG integrated at the $x y l$ locus from ML2297 into ML2000 and selecting 736 on kanamycin + glucose + IPTG. This intermediate strain was then electroporated with pRVMCS737 5-ctrA or pRVMCS-5-ctrA $\Delta 3 \Omega$ respectively selecting on tetracycline + kanamycin + glucose + 738 IPTG.

739 To construct ML3361 and ML3362, ML2000 was first transduced using $\Phi$ Cr30 phage transduction 740 (Ely, 1991) with ML1681 or ML1756 respectively and selected on gentamycin + IPTG plates.

741 These intermediate strains were then electroporated with pQF-podJ and selected on 742 oxytetracycline + gentamycin + IPTG. Strain ML3378 was constructed by electroporating 743 pBXMCS-2-dnaA(R357A) into the same intermediate strain as above from ML2000's transduction 744 with ML1681 and selecting on kanamycin + IPTG + glucose plates. Strain ML3379 was 
745 constructed by electroporating pRVMCS-5-ctrA into ML3378 and selecting on oxytetracycline +

746 kanamycin + IPTG + glucose plates.

747 ML1071 is a lab collection strain. Although the details of this construction are unknown, the linker

748 between ChpT and the C-terminal YFP was sequenced and contains the following sequence:

749 CGGGGCTCGGGCGCCACCATG.

750 ML2000 was transformed with pQF-podJ by electroporation followed by selection on 751 oxytetracycline + IPTG. This intermediate strain was used to construct: ML3363 using $\Phi$ Cr30 752 phage transduction (Ely, 1991) to move ChpT-YFP from ML1071; ML3359 and ML3360 by

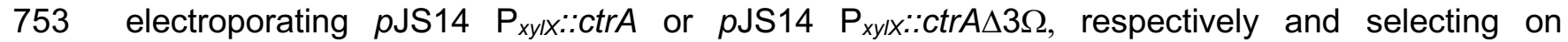
754 oxytetracycline + chloramphenicol + glucose + IPTG plates; ML3367 by electroporating pBVMCS755 4-PA5295 and selecting on oxytetracycline + gentamycin + IPTG plates.

756 To construct ML3370, ML3372 and ML3379, strains ML2395, ML1756 and ML1900 were 757 electroporated with pRVMCS-6-parA(K20R). To construct ML3371, ML3382 and ML3383, ML76 758 was electroporated with pRVMCS-6-parA(K20R) selecting on chloramphenicol plates, and this 759 intermediate strain was then transduced with CckA-eGFP from ML1681 for ML3371, or 760 electroporated with $p X$ XFPC-2-chpT-sfgfp or pXGFPC-2-chpT(R167E)(R169E)(R171E)-sfgfp 761 and selected on chloramphenicol + kanamycin + glucose plates, to construct ML3382 and 762 ML3383 respectively.

763 Strain ML3373 was constructed by electroporating pYFPC-2-chpT-sfgfp into ML76 and selecting 764 on kanamycin plates. Strains ML3374 and ML3375 were constructed by electroporating 765 pRVMCS-6-parA(K20R) or pRVMCS-6-parA, respectively, into ML3373.

766 Strain ML3376 was constructed by electroporating pRVMCS-6-parA into ML2395 and selecting 767 on chloramphenicol + gentamycin plates.

768 Strains ML3377 and ML3378 were constructed by electroporating pRVMCS-6-parA(K20R)-cfp 769 into ML3415 and ML1071 respectively.

770 Strains ML3380 and ML3381 were constructed by electroporating pBXMCS-4-popZ and 771 pBXMCS-2-popZ into ML3374 and ML3371 respectively and selecting on chloramphenicol + 772 gentamycin + kanamycin plates.

773 To construct ML3384, TLS1629 was transduced with ML2397 (MipZ-CFP, $\operatorname{kan}^{\mathrm{R}}$ ) and selected on 774 kanamycin + chloramphenicol plates. 


\section{Time courses and synchronization}

776 Cells were grown overnight in PYE with appropriate antibiotics and inducers (1 mM IPTG for Plac$777 d n a A$ strains) or repressors (0.2\% glucose for xylose inducible constructions). Cells were diluted 778 in $\mathrm{M} 2 \mathrm{G}+$ supplemented with appropriate antibiotics and inducers when necessary and grown to $779 \mathrm{OD}_{600} \sim 0.1$ to 0.4 . For synchronization, cells were first centrifuged for $10 \mathrm{~min}$. at $10,000 \mathrm{~g}$. 780 G1/swarmer cells were isolated using Percoll (GE Healthcare) density gradient centrifugation. 781 Briefly, pellets were resuspended in equal amounts of $\mathrm{M} 2$ buffer $\left(0.87 \mathrm{~g} / \mathrm{liter} \mathrm{Na}_{2} \mathrm{HPO}_{4}, 0.53 \mathrm{~g} / \mathrm{liter}\right.$ $782 \mathrm{KH}_{2} \mathrm{PO}_{4}, 0.5 \mathrm{~g} /$ liter $\mathrm{NH}_{4} \mathrm{Cl}$ ) and Percoll and centrifuged at 10,000 $\mathrm{g}$ for $20 \mathrm{~min}$. The upper ring was 783 aspirated, and the lower ring, corresponding to swarmer cells, was transferred into a new 15-ml

784 Falcon tube. Swarmer cells were washed in $13 \mathrm{ml}$ of M2 buffer and centrifuged for $5 \mathrm{~min}$. at 10,000 $785 \mathrm{~g}$. The pellet was resuspended in $2 \mathrm{ml} \mathrm{M} 2$ buffer and centrifuged for $1 \mathrm{~min}$. at 21,000 g. Cells 786 were released into $\mathrm{M} 2 \mathrm{G}+$ supplemented with appropriate antibiotics (and adjusted to $\mathrm{OD}_{600} \sim 0.05$ 787 to 0.2 when necessary) and the culture was split into different flasks with different inducers. At 788 the indicated time points, samples were harvested from each flask for flow cytometry $(0.15 \mathrm{ml})$, 789 immunoblotting $(1 \mathrm{ml})$, and RNA extraction followed by qRT-PCR $(2 \mathrm{ml})$. For flow cytometry, 790 samples were stored in $30 \%$ ethanol at $4^{\circ} \mathrm{C}$. For immunoblotting and RNA extraction, cells were centrifuged for $1 \mathrm{~min}$. at 15,000 rpm, aspirated, and frozen in liquid nitrogen.

792 For the DnaA depletion strains, prior to synchronization, at $\mathrm{OD}_{600} \sim 0.1$ to 0.25 , cells were 793 centrifuged at $10,000 \mathrm{~g}$ for $10 \mathrm{~min}$, washed 2 times with $\mathrm{M} 2 \mathrm{G}+$, released into $\mathrm{M} 2 \mathrm{G}+$ with 794 appropriate antibiotics without IPTG for 1.5 hours. Cells were then synchronized as described 795 above.

796 For cells induced presynchronization, the culture was split into two flasks and one was induced 797 with $500 \mu \mathrm{M}$ vanillate for the indicated time. $\mathrm{OD}_{600}$ before induction were adjusted to be less than $798 \quad 0.4$ at the end of the induction time. Cells were then synchronized as described above.

\section{$799 \quad$ Flow cytometry}

800 Flow cytometry was performed as described previously (Guzzo et al., 2020). A fraction of fixed 801 cells from the time course sampling (corresponding to an $\mathrm{OD}_{600}$ of $\sim 0.005$ ) were centrifuged at $8026,000 \mathrm{rpm}$ for $4 \mathrm{~min}$. Pelleted cells were resuspended in $1 \mathrm{ml}$ of $\mathrm{Na}_{2} \mathrm{CO}_{3}$ buffer containing $3 \mu \mathrm{g} / \mathrm{ml}$ 803 RNase A (Qiagen) and incubated at $50^{\circ} \mathrm{C}$ for at least $4 \mathrm{~h}$. Cells were supplemented with $0.5 \mu \mathrm{l} / \mathrm{ml}$ 804 SYTOX Green nucleic acid stain (Invitrogen) in $\mathrm{Na}_{2} \mathrm{CO} 3$ buffer and analyzed on a MACSQuant 805 VYB flow cytometer. 


\section{Reverse transcription coupled to quantitative PCR}

807 Reverse transcription coupled to quantitative PCR was performed as described previously (Guzzo

808 et al., 2020). RNA was extracted using hot TRIzol lysis and the Direct-zol RNA miniprep kit 809 (Zymo). 2.5- $\mu$ l of RNA at 50-100 ng/ $\mu$ l was mixed with $0.5 \mu l$ of $100-n g / \mu l$ random hexamer primers 810 (Invitrogen), $0.5 \mu \mathrm{l}$ of $10 \mathrm{mM}$ deoxynucleoside triphosphates (dNTPs) and $3 \mu \mathrm{l}$ of 811 diethylpyrocarbonate (DEPC) water; incubated at $65^{\circ} \mathrm{C}$ for $5 \mathrm{~min}$; and then placed on ice for 1 $812 \mathrm{~min}$. Two microliters of first-strand synthesis buffer, $0.5 \mu \mathrm{l}$ of $100 \mathrm{mM}$ dithiothreitol (DTT), $0.5 \mu \mathrm{l}$ 813 of SUPERase-In (ThermoFisher), and $0.5 \mu$ l of Superscript III (ThermoFisher) were added to each 814 tube, and the following thermocycler program was used: $10 \mathrm{~min}$. at $25^{\circ} \mathrm{C}, 1 \mathrm{~h}$ at $50^{\circ} \mathrm{C}$, and 15 $815 \mathrm{~min}$. at $70^{\circ} \mathrm{C}$. One microliter of RNase $\mathrm{H}(\mathrm{NEB})$ was added to each tube, and each reaction mixture 816 was incubated at $37^{\circ} \mathrm{C}$ for $20 \mathrm{~min}$.

817 cDNA solutions were diluted 10 times in nuclease-free water for quantitative PCR (qPCR). One 818 microliter of diluted cDNA or serially diluted genomic DNA (gDNA) used as a standard curve was 819 mixed with an appropriate pair of primers, i.e., either rpoA_qPCR_1 and rpoA_qPCR_2 as a 820 control, ctrA_qPCR_1 and ctrA_qPCR_5, divK_qPCR_1 and divK_qPCR_2, pdeA_qPCR_3 and 821 pdeA_qPCR_5 or podJ_qPCR_1 and podJ_qPCR_2, and 2X qPCR Master Mix. All experimental 822 samples were loaded as duplicates and with standard curves on a 384-well plate for qPCR. qPCR 823 was conducted in a LightCycler 480 system (Roche) using the following thermocycler program: $82495^{\circ} \mathrm{C}$ for $10 \mathrm{~min}, 95^{\circ} \mathrm{C}$ for $15 \mathrm{~s}, 60^{\circ} \mathrm{C}$ for $30 \mathrm{~s}$, and $72^{\circ} \mathrm{C}$ for $30 \mathrm{~s}$ with 40 cycles of steps 2 to 4 .

\section{Immunoblotting}

826 Immunoblotting was performed as described previously (Guzzo et al., 2020). Frozen pellets from 827 the time course sampling were normalized by $\mathrm{OD}_{600}$ for resuspension in $1 \mathrm{x}$ blue loading buffer 828 (NEB) supplemented with $1 \mathrm{x}$ reducing agent (DTT), boiled at $95^{\circ} \mathrm{C}$ for $10 \mathrm{~min}$, and loaded on $12 \%$ 829 gels (Bio-Rad) for electrophoresis. Proteins were transferred from the gel into polyvinylidene 830 difluoride (PVDF) membranes and immunoblotted. Antibodies were used at the concentrations 831 shown in parentheses: anti-RpoA (1:5,000, BioLegend), anti-CtrA (1:5,000), anti-SciP (1:2000) 832 and anti-GcrA (1:5,000). Horseradish peroxidase (HRP)-conjugated secondary antibodies 833 (ThermoFisher) were used at the concentrations shown in parentheses: anti-mouse $(1: 10,000)$ 834 and anti-rabbit (1:5,000). The membranes were developed with SuperSignal West Femto 835 maximum-sensitivity substrate (ThermoFisher) and visualized with a FluorChem R Imager 836 (ProteinSimple). 


\section{Microscopy}

838 Fixed cells: Cells were grown overnight in PYE with appropriate antibiotics and 1 mM IPTG. Cells

839 were diluted in M2G+ supplemented with appropriate antibiotics and $1 \mathrm{mM}$ IPTG and grown to

$840 \mathrm{OD}_{600} \sim 0.1$ to 0.25 . DnaA was depleted and cells were synchronized as described above (see

841 Time courses and synchronization section). Cells were released into M2G+ supplemented with

842 appropriate antibiotics (and adjusted to $\mathrm{OD}_{600} \sim 0.05$ to 0.2 when necessary) and the culture was

843 split into different flasks with different inducers. At $\mathrm{t}=90 \mathrm{~min}, 1 \mathrm{~mL}$ of culture for each condition

844 was harvested and $25 \mu \mathrm{l}$ of $16 \%$ Paraformaldehyde Aqueous Solution (Fisher Scientific) was

845 added to the cells and gently mixed by inversion. After $1 \mathrm{~min}$. of fixation at room temperature,

846 cells were centrifuged for $1 \mathrm{~min}$. at 10,000 g. Pelleted cells were washed once in $1 \times$ PBS and

847 then resuspended in 1 x PBS. One microliter of cells was spotted onto PBS-1.5\% agarose pads

848 and imaged. Phase-contrast and epifluorescence images were taken on a Zeiss Observer Z1

849 microscope using a 100x/1.4 oil immersion objective and an LED-based Colibri illumination

850 system using MetaMorph software (Universal Imaging, PA).

851 Live imaging: Cells were grown overnight in PYE with appropriate antibiotics and repressor when 852 appropriate $(0.2 \%$ glucose for xylose inducible constructions). Cells were diluted in M2G+ 853 supplemented with appropriate antibiotics and grown to $\mathrm{OD}_{600} \sim 0.1$ to 0.3 . For cells induced 854 presynchronization, the culture was split into two flasks and one was induced with $500 \mu \mathrm{M}$ 855 vanillate for 30 minutes. Cells were then synchronized as described above. After synchronization, 856 cells were released in M2G+ with or without inducer (note that xylose when indicated is added at $857 \mathrm{t}=0$ and not prior to synchronization). Cells were grown in flask for $10 \mathrm{~min}$. and then one microliter 858 of cells was spotted onto M2G+-1.5\% agarose pads containing or not appropriate inducers in a $85935 \mathrm{~mm}$ Glass bottom dish. Phase-contrast and epifluorescence images were taken on a Zeiss 860 Observer Z1 microscope using a 100x/1.4 oil immersion objective and an LED-based Colibri 861 illumination system using MetaMorph software (Universal Imaging, PA). Images were taken every 86210 minutes for up to 250 minutes starting 20 minutes or 30 minutes after synchronization in a $86330^{\circ} \mathrm{C}$ incubation chamber.

\section{Bacterial two-hybrid}

865 Protein interactions were assayed using the bacterial adenylate cyclase two-hybrid system 866 (Karimova et al., 1998). Genes of interest were fused to the 3' or 5' end of the T18 or T25 867 fragments of Bordetella adenylate cyclase using the pUT18C, pUT18, pKT25, or pKNT25 vectors. 868 Different combinations of T18-/T25-fusion plasmids were co-transformed into the BTH101 E. coli 869 strain. Co-transformants were grown in LB supplemented with kanamycin and carbenicillin 
overnight. Saturated cultures were spotted onto MacConkey agar (40 g/L) plates supplemented with maltose (1\%), IPTG $(1 \mathrm{mM})$, kanamycin $\left(25 \mu \mathrm{g} \mathrm{mL}^{-1}\right)$ and carbenicillin $\left(50 \mu \mathrm{g} \mathrm{mL}^{-1}\right)$. Plates were incubated at $30^{\circ} \mathrm{C}$ and pictures taken after two days.

874 Cells were grown overnight in PYE with appropriate antibiotics, $1 \mathrm{mM} \mathrm{IPTG}$ and $0.2 \%$ glucose.

875 Cells were diluted in M2G+ supplemented with appropriate antibiotics and 1 mM IPTG and grown 876 to $\mathrm{OD}_{600} \sim 0.4$. To deplete DnaA, cells were centrifuged at 10,000 $\mathrm{g}$ for $10 \mathrm{~min}$, washed 3 times 877 in M2 and diluted into M2G+ with appropriate antibiotics without IPTG for 2 hours. After the 2878 hour depletion, the cultures $\left(\mathrm{OD}_{600} \sim 0.4\right)$ were synchronized as described above. After 879 synchronization, cells were released into $\mathrm{M} 2 \mathrm{G}+$ and split in different flasks with the indicated 880 inducers. Samples were harvested at the indicated timepoints for genomic DNA (gDNA) 881 extraction. gDNA was extracted by resuspending pellets in $600 \mu \mathrm{L}$ Cell Lysis Solution (QIAGEN) 882 and incubating at $80^{\circ} \mathrm{C}$ for $5 \mathrm{~min}$. to lyse cells. RNAs were removed by treatment with $50 \mu \mathrm{g}$ 883 RNase A (QIAGEN) at $37^{\circ} \mathrm{C}$ for $30 \mathrm{~min}$. $200 \mu \mathrm{L}$ Protein Precipitation Solution (QIAGEN) was 884 added, the sample vortexed, and left on ice for $30 \mathrm{~min}$. to precipitate proteins. After spinning at $88514,000 \mathrm{rpm}$ for $10 \mathrm{~min}$, the supernatant was transferred to a tube containing $600 \mu \mathrm{L}$ isopropanol 886 and mixed by inversion. DNA was harvested by spinning at $14,000 \mathrm{rpm}$ for $1 \mathrm{~min}$. followed by a 887 wash with $600 \mu \mathrm{L}$ of $70 \%$ ethanol. The DNA pellet was resuspended in $50-100 \mu \mathrm{L} \mathrm{H}{ }_{2} \mathrm{O}$. For qPCR, 888 DNAs were diluted 1:500 and mixed with either Cori_2 (chromosomal position: 0 Mb), 889 CCNA_03518 (chr. pos.: $3.676 \mathrm{Mb}$ ), CCNA_00623 (chr. pos.: $0.669 \mathrm{Mb}$ ), CCNA_02846 (chr. pos.: $8902.999 \mathrm{Mb}$ ), CCNA_02567 (chr. pos.: $2.717 \mathrm{Mb})$, CCNA_02189 (2.340 Mb) or CCNA_01869 (chr. 891 pos.: $2.008 \mathrm{Mb}$ ) forward/reverse primer mix and 2X qPCR Master Mix. All experimental samples 892 and standard curves were loaded onto a 384-well plate in duplicate for qPCR. qPCR was 893 conducted in a LightCycler 480 system (Roche) using the following thermocycler program: $95^{\circ} \mathrm{C}$ 894 for $10 \mathrm{~min}, 95^{\circ} \mathrm{C}$ for $15 \mathrm{~s}, 60^{\circ} \mathrm{C}$ for $30 \mathrm{~s}$, and $72^{\circ} \mathrm{C}$ for $30 \mathrm{~s}$ with 40 cycles of steps 2 to 4.

\section{Plasmid construction}

896 Integration plasmids

897 pYFPC-2-chpTsfGFP: the pYFPC-2 plasmid (Thanbichler et al., 2007) was amplified using 898 primers pYFPC2_chpT_up_R and sfGFP_dwn_pYFPC_2_F and the chpT gene was amplified 899 using primers pYFPC2_chpT_up_F and chpT_dwn_sfGFP_up_R. The chpT PCR product, a 900 sfGFP gblock codon optimized for Caulobacter from IDT, and the PCR fragment of the plasmid 901 were assembled together using the Hifi DNA assembly mix (NEB). The construction was verified 902 by sequencing. 
pXGFPC-2-chpT-sfGFP: The chpT-sfGFP insert was amplified from pYFPC-2-chpT-sfgfp using primers pBX_chpT_up_F and sfGFP_pXGFPC_down_R2. The pXGFPC-2 plasmid (Thanbichler et al., 2007) was amplified using primers sfGFP_pXGFPC_down_F2 and pBX_chpT_up_R. The two PCR products were assembled together using the Hifi DNA assembly mix (NEB). The construction was verified by sequencing.

908 pXGFPC-2-chpT(R167E)(R169E)(R171E)-sfGFP: same cloning protocol as pXGFPC-2-chpTsfGFP but using pKNT25-ChpT(R167E)(R169E)(R171E)-T25 as a template. The construction was verified by sequencing.

911 Replicative plasmids

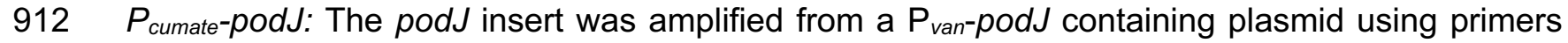
913 podJ_4pQF_1..24 and podJ_4pQF_last24 and. The pQF plasmid (Kaczmarczyk et al., 2013) was 914 amplified using primers pQF_CPEC_up and pQF_CPEC_noFLAG_down. The two PCR products 915 were assembled together using circular polymerase extension cloning (CPEC). The construction 916 was verified by sequencing.

917 pRVMCS-5-ctrA : This plasmid was cloned by first amplifying ctrA from genomic DNA using 918 primers ctrA-Ndel-F and ctrA-extrastop-Nhel-R. The PCR product was digested with Ndel and 919 Nhel-HF and ligated into similarly digested pRVMCS-5 plasmid (Thanbichler et al., 2007) using 920 T4 DNA ligase. The construction was verified by sequencing.

921 pRVMCS-5-ctrA $\triangle 3 \Omega$ : This plasmid was cloned using the same protocol as for $p R V M C S-5-$ 922 ctrA but by amplifying ctrA from ML46 using primers ctrA-Ndel-F and ctrAD3W-Nhel-R. The 923 construction was verified by sequencing.

$924 p B X M C S-2-d n a A(R 357 A)$ : This plasmid was cloned by first using around-the-horn PCR to 925 introduce the R357A mutation into $d n a A$ (from a $\mathrm{P}_{v a n}-d n a A$ containing plasmid) using primers 926 dnaA 1068..1048 and dnaA 1069..1095 R357A. The dnaA(R357A) allele was then amplified using 927 primers DnaA_rev_3xTAA_EcoRI and Ndel_DnaA_1..23 and cloned into pBXMCS-2 928 (Thanbichler et al., 2007) using EcoRI/Ndel. The construction was verified by sequencing.

929 pRVMCS-6-parA(K20R): genomic DNA from ML2414 (Badrinarayanan et al., 2015) was used as 930 a template to amplify $\operatorname{parA}(K 20 R)$ using primers pXYFP_ParA_Ndel_up_F and 931 ParA_TAATAA_Sacl_R. The PCR product was digested with Ndel and Sacl restriction enzymes 932 and ligated into similarly digested pRVMCS-6 plasmid (Thanbichler et al., 2007) using T4 DNA 933 ligase. The construction was verified by sequencing. 
934 pRVMCS-6-parA: the parA gene was amplified from pKT25-parA using primers 935 pRVMCS6_parA_up_F and pRVMCS-6_parA_dwn_R and the pRVMCS-6-parA(K20R) plasmid 936 template was amplified in two separate fragments using primers pRVMCS6_parA_dwn_F and 937 pRVMCS6_upcat_R, and pRVMCS6_parA_up_R and pRMCS_mid_F. The parA fragment and 938 the two plasmid fragments were assembled using the Hifi DNA assembly mix (NEB). The 939 construction was verified by sequencing.

940 pRVMCS-6-parA(K20R)-CFP: the parA(K20R) fragment was amplified from pRVMCS-6941 parA(K20R) using primers pRVMCS-6-parA_up_F and parA_CFP_R. The cfp fragment was 942 amplified from pXCFPC-6 (Thanbichler et al., 2007) using primers parA_CFP_F and 943 CFP_pRVMCS-6_dwn_R. The pRVMCS-6-parA(K20R) plasmid template was amplified in two 944 separate fragments using primers and CFP_pRVMCS-6_dwn_F and pRVMCS6_upcat_R, and 945 pRVMCS6_parA_up_R and pRMCS_mid_F. The parA(K20R) fragment, the cfp insert and the two 946 plasmid fragments were assembled using the Hifi DNA assembly mix (NEB). The construction 947 was verified by sequencing.

948 pBXMCS-2-popZ and pBXMCS-4-popZ: The popZ insert was amplified from genomic DNA using 949 pBX_popZ_up_F and pB_popZ_down_R and the pBXMCS-2 or pBXMCS-4 (Thanbichler et al., 950 2007) plasmids were amplified using primers pB_popZ_down_F and pBX_popZ_up_R. The popZ 951 insert and the plasmid PCR product were assembled together using the Hifi DNA assembly mix 952 (NEB). The construction was verified by sequencing.

953 Bacterial two-hybrid plasmids

954 All bacterial two-hybrid plasmids were constructed using the Gibson assembly mix (NEB) or the 955 Hifi DNA assembly mix (NEB). All constructions (gene of interest and T18- or T25-fragments) 956 were verified by sequencing.

957 pKNT25-ChpT: The chpT fragment was amplified using primers pKNT25_chpT_up_F and 958 pKNT25_chpT_down_R. The pKNT25 plasmid was amplified using primers pKNT25_chpT_up_R 959 and pKNT25_chpT_down_F.

960 pKNT25-ChpT $T_{\text {DHP }}$ : The $c h p T_{D H P}$ fragment was amplified using primers pKNT25_chpT_up_F and 961 pKNT25_chpTDHP_down_R. The pKNT25 plasmid was amplified using primers 962 pKNT25_chpT_up_R and pKNT25_chpTDHP_down_F.

963 pKT25-CckAн: The $c c k A_{H K}$ fragment was amplified using primers pKT25_cckA_HK_up_F and 964 pKT25_cckA_HK_down_R. The pKT25 plasmid was amplified using primers pKT25_up_R and 965 pKT25_down_F. 
pKNT25-ChpT(R167E)(R169E)(R171E)-T25 : Using pKNT25-ChpT as a template, the plasmid was amplified using primers R167E169E171E_short2_F and R167E169E171E_short2_R. The two R167E169E171E ultramers (forward and reverse) were mixed together in equal molar amounts and duplexed using the following thermocycler program: $94^{\circ} \mathrm{C}$ for 3 minutes, cool down to $25^{\circ} \mathrm{C}$ over 45 minutes at a pace of $1.5^{\circ} \mathrm{C}$ per minute. The duplexed ultramers were diluted 100 -

971 fold before addition to the Gibson reaction with the plasmid fragment.

972 pUT18C-ParB: The parB fragment was amplified using primers pUT18C_parB_up_F and 973 pUT18C_parB_down_R. The pUT18C plasmid was amplified using primers pUT18C_parB_up_R 974 and pUT18C_parB_down_F.

975 pUT18C-ParA and pUT18C-ParA(K20R): The parA or parA(K20R) fragment were amplified using 976 primers pUT18C_parA_up_F and pUT18C_parA_down_R. The pUT18C plasmid was amplified 977 using primers pUT18C_parA_up_R and pUT18C_parA_down_F.

978 pUT18C-ParA(R195E): Using pUT18C-ParA as a template, the plasmid was amplified using 979 primers ParAR195E_F and ParAR195E_R. The two ParA(R195E) ultramers (forward and 980 reverse) were duplexed and used as described for pKNT25-ChpT(R167E)(R169E)(R171E)-T25.

981 pUT18C-ParA(K20R)(R195E): Using pUT18C-ParA(K20R) as a template, the plasmid was 982 amplified using primers ParAR195E_F and ParAR195E_R. The two ParA(R195E) ultramers 983 (forward and reverse) were duplexed and used as described for pKNT25984 ChpT(R167E)(R169E)(R171E)-T25.

985 pUT18C-ParA(D44A): Using pUT18C-ParA as a template, the plasmid was amplified using primers ParAD44A_F and ParAD44A_R. The two ParA(D44A) ultramers (forward and reverse) were duplexed and used as described for pKNT25-ChpT(R167E)(R169E)(R171E)-T25. pUT18C-ParA(D44A)(R195E): Using pUT18C-ParA(R195E) as a template, the plasmid was amplified using primers ParAD44A_F and ParAD44A_R. The two ParA(D44A) ultramers (forward 990 and reverse) were duplexed and used as described for pKNT25-ChpT(R167E)(R169E)(R171E)991 T25.

992 pUT18C-ParA(G16V): Using pUT18C-ParA as a template, the plasmid was amplified using 993 primers ParAG16V_F and ParAG16V_R. The two ParA(G16V) ultramers (forward and reverse) 994 were duplexed and used as described for pKNT25-ChpT(R167E)(R169E)(R171E)-T25. 
995 pUT18-ChpT: The chpT fragment was amplified using primers pUT18_chpT_up_F and 996 pUT18_chpT_down_R. The pUT18 plasmid was amplified using primers pUT18_chpT_up_R and 997 pUT18_chpT_down_F.

998 pUT18C-CckA $A_{R D}$ : The cckA $A_{R D}$ fragment was amplified using primers pUT18C_cckA_RD_up_F 999 and pUT18C_cckA_RD_down_R. The pUT18C plasmid was amplified using primers 1000 pUT18C_cckA_RD_up_R and pUT18C_cckA_RD_down_F.

1001 pUT18C-MipZ: The mipZ fragment was amplified using primers pUT18C_mipZ_up_F and 1002 pUT18C_mipZ_down_R. The pUT18C plasmid was amplified using primers pUT18C_mipZ_up_R 1003 and pUT18C_mipZ_down_F.

1004 pUT18C-MipZ(R194A): this plasmid was first constructed by inserting the R194A mutation into 1005 mipZ in the pKNT25-mipZ plasmid using primers mipZR194A_F and mipZR194A_R. The two 1006 MipZ(R194A) ultramers (forward and reverse) were duplexed and used as described for $p K N T 25-$ 1007 ChpT(R167E)(R169E)(R171E)-T25. The pUT18C-MipZ(R194A) plasmid was then constructed by 1008 amplifying the mipZ(R194A) fragment from pKNT25-mipZ(R194A) using primers 1009 pUT18C_mipZ_up_F and pUT18C_mipZ_down_R and the pUT18C plasmid was amplified using 1010

1011 Quantification and statistical analysis

1012 qRT-PCR

1013 Crossing point (Cp) values were calculated from LightCycler 480 software at the second derivative 1014 maximum. Technical replicates were averaged to yield a final $\mathrm{Cp}$ value for each sample and 1015 relative quantities of cDNA were calculated based on a 3-fold dilution standard curve. Each time 1016 point value for each gene of interest was normalized to the rpoA measured value, as $r p o A$ 1017 expression remains constant in exponential phase.

1018 Dose response fold-differences at timepoint 100 minutes are the ratios between the normalized 1019 cDNA relative quantity of the induce condition and the non-induced condition. Independent 1020 biological replicates individual datapoints are shown as well as the average ratio.

1021 qPCR/origin-ter ratio

$1022 \mathrm{Cp}$ values were calculated from LightCycler 480 software at the second derivative maximum. 1023 Technical replicates were averaged to yield a final $\mathrm{Cp}$ value for each sample and standard curve 1024 point. Relative quantities of cDNA were calculated based on a 3-fold dilution standard curve. 1025 Chromosome copy numbers were normalized internally to CCNA_01869 (terminus region) as a 
1026 loading control and then normalized to the origin abundance after synchrony ( 0 min) to evaluate

1027 fold-change from $1 \mathrm{~N}$.

\section{Quantification of protein levels by immunoblotting}

1029 Image quantification and analysis was done with Fiji/lmageJ. Band intensities were measured for 1030 each protein. RpoA band intensities were used as a loading control for each sample, meaning 1031 that each protein band intensity in a specific lane were normalized to RpoA band intensity in that 1032 same lane.

\section{Flow cytometry analysis}

1034 Flow cytometry was analyzed with FlowJo from 50,000 total SYTOX positive events for each 1035 experiment. For each experiment, 1N was determined based on the distribution of $\mathrm{G} 1$ cells and $10362 \mathrm{~N}$ based on the control condition.

\section{Microscopy}

1038 Polar clusters were counted manually or using MicrobeJ. The swarmer pole was determined by 1039 identifying the stalk on phase contrast images. The percentage of cells with polar clusters at the 1040 new pole were calculated for a total number of cells as specified in the figures using at least two 1041 biological replicates for each condition. For the live microscopy, a cell was counted as containing 1042 a polar cluster at the swarmer pole or an internal cluster if a cluster was identified at any time 1043 during the course of the time-lapse. Kymographs were built using Fiji and the time lapse alignment 1044 were made using the "Straighten" function in Fiji. The fluorescence intensities from the old pole 1045 to the new pole were measured automatically using MicrobeJ. For quantifying the percentage of 1046 elongated cells in strains expressing parA(K20R) and MipZ, DivL, CckA, or ChpT fused to a 1047 fluorescent protein, we examined cells from two independent cultures. 


\section{References}

Abel, S., Bucher, T., Nicollier, M., Hug, I., Kaever, V., Abel zur Wiesch, P. and Jenal, U. (2013) 'Bi-modal Distribution of the Second Messenger c-di-GMP Controls Cell Fate and Asymmetry during the Caulobacter Cell Cycle', PLoS Genetics, 9(9), pp. 5-11. doi: 10.1371/journal.pgen.1003744.

Angelastro, P. S., Sliusarenko, O. and Jacobs-Wagner, C. (2010) 'Polar localization of the CckA histidine kinase and cell cycle periodicity of the essential master regulator CtrA in Caulobacter crescentus', Journal of Bacteriology, 192(2), pp. 539-552. doi: 10.1128/JB.00985-09. homologous loci enables double-strand break repair in bacteria', Journal of Cell Biology, 210(3), pp. 385-400. doi: 10.1083/jcb.201505019.

Banani, S. F., Lee, H. O., Hyman, A. A. and Rosen, M. K. (2017) 'Biomolecular condensates: Organizers of cellular biochemistry', Nature Reviews Molecular Cell Biology. Nature Publishing Group, pp. 285-298. doi: 10.1038/nrm.2017.7.

Bernhardt, T. G. and De Boer, P. A. J. (2005) 'SImA, a nucleoid-associated, FtsZ binding protein required for blocking septal ring assembly over chromosomes in E. coli', Molecular Cell, 18(5), pp. 555-564. doi: 10.1016/j.molcel.2005.04.012.

1066 Biondi, E. G., Reisinger, S. J., Skerker, J. M., Arif, M., Perchuk, B. S., Ryan, K. R. and Laub, M. 1067 T. (2006) 'Regulation of the bacterial cell cycle by an integrated genetic circuit', Nature, 1068 444(7121), pp. 899-904. doi: 10.1038/nature05321.

1069 Blair, J. A., Xu, Q., Childers, W. S., Mathews, I. I., Kern, J. W., Eckart, M., Deacon, A. M. and 1070 Shapiro, L. (2013) 'Branched signal wiring of an essential bacterial cell-cycle phosphotransfer 1071 protein', Structure. doi: 10.1016/j.str.2013.06.024.

1072 Bowman, G. R. et al. (2010) 'Caulobacter PopZ forms a polar subdomain dictating sequential 1073 changes in pole composition and function', Molecular Microbiology, 76(1), pp. 173-189. doi: 1074 10.1111/j.1365-2958.2010.07088.x.

1075 Chen, Y. E., Tsokos, C. G., Biondi, E. G., Perchuk, B. S. and Laub, M. T. (2009) 'Dynamics of 1076 two phosphorelays controlling cell cycle progression in Caulobacter crescentus', Journal of 1077 Bacteriology, 191(24), pp. 7417-7429. doi: 10.1128/JB.00992-09.

1078 Chien, P., Perchuk, B. S., Laub, M. T., Sauer, R. T. and Baker, T. A. (2007) 'Direct and adaptor1079 mediated substrate recognition by an essential AAA+ protease', Proceedings of the National 
Academy of Sciences of the United States of America, 104(16), pp. 6590-6595. doi: 10.1073/pnas.0701776104.

1082 Corrales-Guerrero, L., He, B., Refes, Y., Panis, G., Bange, G., Viollier, P. H., Steinchen, W. and Thanbichler, M. (2020) 'Molecular architecture of the DNA-binding sites of the P-loop ATPases MipZ and ParA from Caulobacter crescentus', Nucleic acids research, 48(9), pp. 4769-4779. doi: 10.1093/nar/gkaa192.

1086

Curtis, P. D., Quardokus, E. M., Lawler, M. L., Guo, X., Klein, D., Chen, J. C., Arnold, R. J. and Brun, Y. V. (2012) 'The scaffolding and signalling functions of a localization factor impact polar development', Molecular Microbiology, 84(4), pp. 712-735. doi: 10.1111/j.13652958.2012.08055.x.

1090 Domian, I. J., Quon, K. C. and Shapiro, L. (1997) 'Cell Type-Specific Phosphorylation and Proteolysis of a Transcriptional Regulator Controls the G1-to-S Transition in a Bacterial Cell Cycle', Cell, 90(3), pp. 415-424. doi: 10.1016/S0092-8674(00)80502-4.

Ebersbach, G., Briegel, A., Jensen, G. J. and Jacobs-Wagner, C. (2008) 'A Self-Associating Protein Critical for Chromosome Attachment, Division, and Polar Organization in Caulobacter', Cell, 134(6), pp. 956-968. doi: 10.1016/j.cell.2008.07.016.

Ely, B. (1991) '[17] Genetics of Caulobacter crescentus', Methods in Enzymology, 204, pp. 372384. doi: 10.1016/0076-6879(91)04019-K.

1098 Fernandez-Fernandez, C., Gonzalez, D. and Collier, J. (2011) 'Regulation of the activity of the dual-function DnaA protein in Caulobacter crescentus', PLoS ONE, 6(10). doi: 10.1371/journal.pone.0026028.

1101 Fioravanti, A., Clantin, B., Dewitte, F., Lens, Z., Verger, A., Biondi, E. G. and Villeret, V. (2012) 1102 'Structural insights into ChpT, an essential dimeric histidine phosphotransferase regulating the cell cycle in Caulobacter crescentus', Acta Crystallographica Section F: Structural Biology and Crystallization Communications, 68(9), pp. 1025-1029. doi: 10.1107/S1744309112033064. 7127. doi: 10.1074/jbc.TM118.001192.

1108 Gora, K. G., Tsokos, C. G., Chen, Y. E., Srinivasan, B. S., Perchuk, B. S. and Laub, M. T. (2010) 1109 'A cell-type-specific protein-protein interaction modulates transcriptional activity of a master 1110 regulator in caulobacter crescentus', Molecular Cell, 39(3), pp. 455-467. doi: 
1112 Gorbatyuk, B. and Marczynski, G. T. (2001) 'Physiological consequences of blocked Caulobacter 1113 crescentus dnaA expression, an essential DNA replication gene', Molecular Microbiology, 40(2), 1114 pp. 485-497. doi: 10.1046/j.1365-2958.2001.02404.x.

1115 Gorbsky, G. J. (2015) 'The spindle checkpoint and chromosome segregation in meiosis', FEBS 1116 Journal, 282(13), pp. 2471-2487. doi: 10.1111/febs.13166.

1117 Gruber, S. and Errington, J. (2009) 'Recruitment of Condensin to Replication Origin Regions by 1118 ParB/SpoOJ Promotes Chromosome Segregation in B. subtilis', Cell, 137(4), pp. 685-696. doi: 1119 10.1016/j.cell.2009.02.035.

1120 Guzzo, M., Castro, L. K., Reisch, C. R., Guo, M. S. and Laub, M. T. (2020) 'A CRISPR Interference 1121 System for Efficient and Rapid Gene Knockdown in Caulobacter crescentus', 11(1), pp. 1-16.

1122 Haakonsen, D. L., Yuan, A. H. and Laub, M. T. (2015) 'The bacterial cell cycle regulator GcrA is 1123 a $\sigma 70$ cofactor that drives gene expression from a subset of methylated promoters.', Genes \& 1124 development, 29(21), pp. 2272-86. doi: 10.1101/gad.270660.115.

1125 Hartwell, L. H. and Weinert, T. A. (1989) 'Checkpoints: Controls that ensure the order of cell cycle 1126 events', Science, 246(4930), pp. 629-634. doi: 10.1126/science.2683079.

1127 Holmes, J. A., Follett, S. E., Wang, H., Meadows, C. P., Varga, K. and Bowman, G. R. (2016) 1128 'Caulobacter PopZ forms an intrinsically disordered hub in organizing bacterial cell poles ', 1129 Proceedings of the National Academy of Sciences, 113(44), pp. 12490-12495. doi: 1130 10.1073/pnas.1602380113.

1131 Holtzendorff, J., Holtzendorff, J., Hung, D., Brende, P., Reisenauer, A., Viollier, P. H., Mcadams, 1132 H. H. and Shapiro, L. (2004) 'Oscillating global regulators control the genetic circuit driving a 1133 bacterial cell cycle.', Science, 304(2004), pp. 10-15. doi: 10.1126/science.1095191.

1134 Hottes, A. K., Shapiro, L. and McAdams, H. H. (2005) ‘DnaA coordinates replication initiation and 1135 cell cycle transcription in Caulobacter crescentus', Molecular Microbiology, 58(5), pp. 1340-1353. 1136 doi: 10.1111/j.1365-2958.2005.04912.x.

1137 Iniesta, A. A., Hillson, N. J. and Shapiro, L. (2010a) 'Cell pole-specific activation of a critical 1138 bacterial cell cycle kinase', Proceedings of the National Academy of Sciences, 107(15), pp. 70121139 7017. doi: 10.1073/pnas.1001767107.

1140 Iniesta, A. A., Hillson, N. J. and Shapiro, L. (2010b) 'Polar remodeling and histidine kinase 1141 activation, which is essential for Caulobacter cell cycle progression, are dependent on DNA 
1142 replication initiation', Journal of Bacteriology, 192(15), pp. 3893-3902. doi: 10.1128/JB.00468-10.

1143 Iniesta, A. A., McGrath, P. T., Reisenauer, A., McAdams, H. H. and Shapiro, L. (2006) 'A phospho1144 signaling pathway controls the localization and activity of a protease complex critical for bacterial 1145 cell cycle progression', Proceedings of the National Academy of Sciences, 103(29), pp. 109351146 10940. doi: 10.1073/pnas.0604554103.

1147 Jacobs, C., Domian, I. J., Maddock, J. R. and Shapiro, L. (1999) 'Cell cycle-dependent polar 1148 localization of an essential bacterial histidine kinase that controls DNA replication and cell 1149 division', Cell, 97(1), pp. 111-120. doi: 10.1016/S0092-8674(00)80719-9.

1150 Jalal, A. S., Tran, N. T. and Le, T. B. (2020) 'ParB spreading on DNA requires cytidine 1151 triphosphate in vitro', eLife, 9, pp. 1-24. doi: 10.7554/eLife.53515.

1152 Jenal, U. and Fuchs, T. (1998) 'An essential protease involved in bacterial cell-cycle control', 1153 EMBO Journal, 17(19), pp. 5658-5669. doi: 10.1093/emboj/17.19.5658.

1154 Jonas, K., Chen, Y. E. and Laub, M. T. (2011) 'Modularity of the bacterial cell cycle enables 1155 independent spatial and temporal control of DNA replication', Current Biology, 21(13), pp. 10921156 1101. doi: 10.1016/j.cub.2011.05.040.

1157 Kaczmarczyk, A., Vorholt, J. A. and Francez-Charlot, A. (2013) 'Cumate-inducible gene 1158 expression system for sphingomonads and other Alphaproteobacteria', Applied and 1159 Environmental Microbiology, 79(21), pp. 6795-6802. doi: 10.1128/AEM.02296-13.

1160 Karimova, G., Pidoux, J., Ullmann, A. and Ladant, D. (1998) 'A bacterial two-hybrid system based on a reconstituted signal transduction pathway', Proceedings of the National Academy of Sciences of the United States of America, 95(10), pp. 5752-5756. doi: 10.1073/pnas.95.10.5752.

1163 Kawalek, A., Wawrzyniak, P., Bartosik, A. A. and Jagura-Burdzy, G. (2020) 'Rules and 1164 exceptions: The role of chromosomal ParB in DNA segregation and other cellular processes', 1165 Microorganisms. MDPI AG, p. 105. doi: 10.3390/microorganisms8010105.

1166 Laloux, G. and Jacobs-Wagner, C. (2013) 'Spatiotemporal control of PopZ localization through 1167 cell cycle-coupled multimerization', Journal of Cell Biology, 201(6), pp. 827-841. doi: 1168 10.1083/jcb.201303036.

1169 Lasker, K., von Diezmann, L., Zhou, X., Ahrens, D. G., Mann, T. H., Moerner, W. E. and Shapiro, 1170 L. (2020) 'Selective sequestration of signalling proteins in a membraneless organelle reinforces 1171 the spatial regulation of asymmetry in Caulobacter crescentus', Nature Microbiology. Nature 1172 Research, pp. 418-429. doi: 10.1038/s41564-019-0647-7. 
1173 Laub, M. T., Chen, S. L., Shapiro, L. and McAdams, H. H. (2002) 'Genes directly controlled by

1174 CtrA, a master regulator of the Caulobacter cell cycle', Proceedings of the National Academy of

1175 Sciences, 99(7), pp. 4632-4637. doi: 10.1073/pnas.062065699.

1176 Laub, M. T., Perlmann, T., Jansson, L. and Dev, G. (2000) 'Global Analysis of the Genetic Network

1177 Controlling a Bacterial Cell Cycle', Science, 2144(2000), pp. 2144-2149. doi:

1178 10.1126/science.290.5499.2144.

1179 Lim, H. C., Surovtsev, I. V., Beltran, B. G., Huang, F., Bewersdorf, J. and Jacobs-Wagner, C. 1180 (2014) 'Evidence for a DNA-relay mechanism in ParABS-mediated chromosome segregation', 1181 eLife, 2014(3). doi: 10.7554/eLife.02758.

1182 Lori, C., Ozaki, S., Steiner, S., Böhm, R., Abel, S., Dubey, B. N., Schirmer, T., Hiller, S. and Jenal, 1183 U. (2015) 'Cyclic di-GMP acts as a cell cycle oscillator to drive chromosome replication', Nature, 1184 523(7559), pp. 236-239. doi: 10.1038/nature14473.

1185 Marczynski, G. T. (1999) 'Chromosome methylation and measurement of faithful, once and only 1186 once per cell cycle chromosome replication in Caulobacter crescentus', Journal of Bacteriology, 1187 181(7), pp. 1984-1993. doi: 10.1128/jb.181.7.1984-1993.1999.

1188 Mercy, C. et al. (2019) 'RocS drives chromosome segregation and nucleoid protection in 1189 Streptococcus pneumoniae', Nature Microbiology, 4(10), pp. 1661-1670. doi: 10.1038/s415641190 019-0472-z.

1191 Murray, A. W. (1992) 'Creative blocks: Cell-cycle checkpoints and feedback controls', Nature. 1192 Nature Publishing Group, pp. 599-604. doi: 10.1038/359599a0.

1193 Perego, M. (2013) 'Forty Years in the Making: Understanding the Molecular Mechanism of 1194 Peptide Regulation in Bacterial Development', PLoS Biology, 11(3), p. e1001516. doi: 1195 10.1371/journal.pbio.1001516.

1196 Pióro, M. and Jakimowicz, D. (2020) ‘Chromosome Segregation Proteins as Coordinators of Cell 1197 Cycle in Response to Environmental Conditions', Frontiers in Microbiology. Frontiers Media S.A. 1198 doi: 10.3389/fmicb.2020.00588.

1199 Ptacin, J. L., Gahlmann, A., Bowman, G. R., Perez, A. M., von Diezmann, A. R. S., Eckart, M. R., 1200 Moerner, W. E. and Shapiro, L. (2014) 'Bacterial scaffold directs pole-specific centromere 1201 segregation', Proceedings of the National Academy of Sciences, 111(19), pp. E2046-E2055. doi: 1202 10.1073/pnas.1405188111.

1203 Ptacin, J. L., Lee, S. F., Garner, E. C., Toro, E., Eckart, M., Comolli, L. R., Moerner, W. E. and 
1204 Shapiro, L. (2010) 'A spindle-like apparatus guides bacterial chromosome segregation', Nature 1205 Cell Biology, 12(8), pp. 791-798. doi: 10.1038/ncb2083.

1206 Quon, K. C., Yang, B., Domian, I. J., Shapiro, L. and Marczynski, G. T. (1998) 'Negative control 1207 of bacterial DNA replication by a cell cycle regulatory protein that binds at the chromosome origin', 1208 Proceedings of the National Academy of Sciences, 95(1), pp. 120-125. doi: 1209 10.1073/PNAS.95.1.120.

1210 Rudner, D. Z. and Losick, R. (2001) 'Morphological Coupling in Development: Lessons from 1211 Prokaryotes', Developmental Cell. Cell Press, pp. 733-742. doi: 10.1016/S1534-5807(01)0009412126.

1213 Schofield, W. B., Lim, H. C. and Jacobs-Wagner, C. (2010) 'Cell cycle coordination and regulation 1214 of bacterial chromosome segregation dynamics by polarly localized proteins', EMBO Journal, 1215 29(18), pp. 3068-3081. doi: 10.1038/emboj.2010.207.

1216 Shebelut, C. W., Guberman, J. M., van Teeffelen, S., Yakhnina, A. A. and Gitai, Z. (2010) 1217 'Caulobacter chromosome segregation is an ordered multistep process', Proceedings of the 1218 National Academy of Sciences, 107(32), pp. 14194-14198. doi: 10.1073/pnas.1005274107.

1219 Solomon, J. M., Lazazzera, B. A. and Grossman, A. D. (1996) 'Purification and characterization of an extracellular peptide factor that affects two different developmental pathways in Bacillus subtilis', Genes and Development, 10(16), pp. 2014-2024. doi: 10.1101/gad.10.16.2014.

1222 Storchova, Z. and Pellman, D. (2004) 'From polyploidy to aneuploidy, genome instability and cancer', Nature Reviews Molecular Cell Biology. Nature Publishing Group, pp. 45-54. doi: 10.1038/nrm1276.

Thanbichler, M., Iniesta, A. A. and Shapiro, L. (2007) 'A comprehensive set of plasmids for vanillate- and xylose-inducible gene expression in Caulobacter crescentus.', Nucleic acids research, 35(20), p. e137. doi: 10.1093/nar/gkm818.

1228 Thanbichler, M. and Shapiro, L. (2006) 'MipZ, a Spatial Regulator Coordinating Chromosome 1229 Segregation with Cell Division in Caulobacter', Cell, 126(1), pp. 147-162. doi: $1230 \quad 10.1016 /$ j.cell.2006.05.038.

1231 Toro, E., Hong, S.-H., McAdams, H. H. and Shapiro, L. (2008) 'Caulobacter requires a dedicated 1232 mechanism to initiate chromosome segregation', Proceedings of the National Academy of 1233 Sciences, 105(40), pp. 15435-15440. doi: 10.1073/pnas.0807448105.

1234 Tran, N. T., Stevenson, C. E., Som, N. F., Thanapipatsiri, A., Jalal, A. S. B. and Le, T. B. K. (2018) 
1235 'Permissive zones for the centromere-binding protein ParB on the Caulobacter

1236 crescentuschromosome', Nucleic Acids Research, 46(3), pp. 1196-1209. doi:

1237 10.1093/nar/gkx1192.

1238 Tsokos, C. G., Perchuk, B. S. and Laub, M. T. (2011) 'A Dynamic Complex of Signaling Proteins

1239 Uses Polar Localization to Regulate Cell-Fate Asymmetry in Caulobacter crescentus',

1240 Developmental Cell, 20(3), pp. 329-341. doi: 10.1016/j.devcel.2011.01.007.

1241 Wortinger, M., Sackett, M. J. and Brun, Y. V. (2000) 'CtrA mediates a DNA replication checkpoint

1242 that prevents cell division in Caulobacter crescentus', EMBO Journal, 19(17), pp. 4503-4512. doi:

1243 10.1093/emboj/19.17.4503.

1244 Wu, L. J. and Errington, J. (2004) 'Coordination of cell division and chromosome segregation by

1245 a nucleoid occlusion protein in Bacillus subtilis', Cell, 117(7), pp. 915-925. doi: 1246 10.1016/j.cell.2004.06.002. 
1245 'Permissive zones for the centromere-binding protein ParB on the Caulobacter

1246 crescentuschromosome', Nucleic Acids Research, 46(3), pp. 1196-1209. doi:

$1247 \quad 10.1093 /$ nar/gkx1192.

1248 Tsokos, C. G., Perchuk, B. S. and Laub, M. T. (2011) 'A Dynamic Complex of Signaling Proteins

1249 Uses Polar Localization to Regulate Cell-Fate Asymmetry in Caulobacter crescentus', 1250 Developmental Cell, 20(3), pp. 329-341. doi: 10.1016/j.devcel.2011.01.007.

1251 Wortinger, M., Sackett, M. J. and Brun, Y. V. (2000) 'CtrA mediates a DNA replication checkpoint 1252 that prevents cell division in Caulobacter crescentus', EMBO Journal, 19(17), pp. 4503-4512. doi: 1253 10.1093/emboj/19.17.4503.

1254 Wu, L. J. and Errington, J. (2004) 'Coordination of cell division and chromosome segregation by 1255 a nucleoid occlusion protein in Bacillus subtilis', Cell, 117(7), pp. 915-925. doi: 1256 10.1016/j.cell.2004.06.002. 
bioRxiv preprint doi: https://doi.org/10.1101/2021.03.08.434431; this version posted March 8, 2021. The copyright holder for this preprint (which was not certified by peer review) is the author/funder, who has granted bioRxiv a license to display the preprint in perpetuity. It is mad available under aCC-BY-NC-ND 4.0 International license.

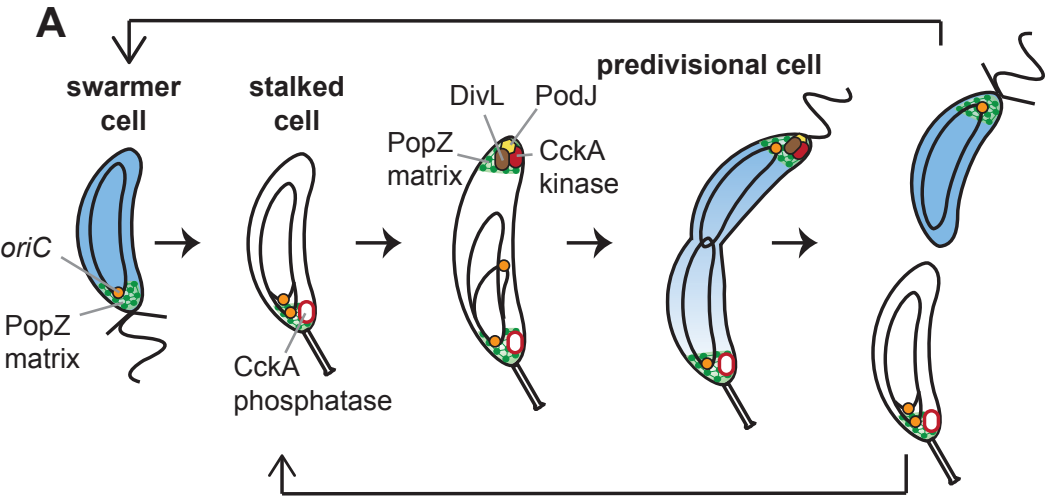

active CtrA

B

\begin{tabular}{|c|c|c|c|c|c|c|c|c|}
\hline \multirow[b]{2}{*}{0} & \multicolumn{2}{|r|}{$+d n a A$} & \multicolumn{2}{|r|}{ - dnaA } & \multicolumn{3}{|c|}{$\begin{array}{r}-\operatorname{dna} A \\
+\operatorname{ctrA}\end{array}$} & $\begin{array}{c}-d n a A \\
+\operatorname{ctr} A+\text { podJ }\end{array}$ \\
\hline & 40 & $100 \quad 120$ & 40 & 100120 & 40 & 100 & 120 & $100 \quad 120$ \\
\hline
\end{tabular}
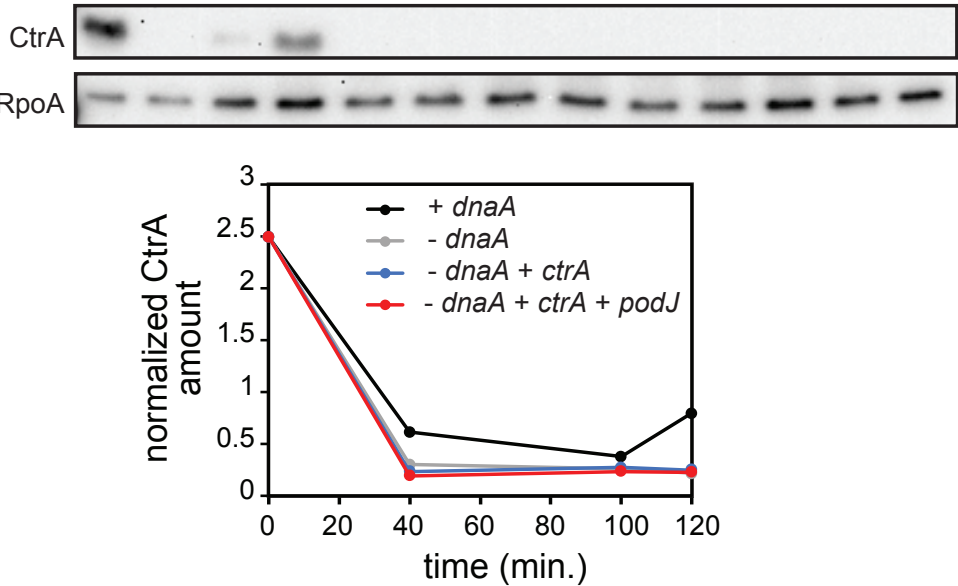

C

$\mathrm{P}_{\text {lac }}-$ dnaA, $\mathrm{P}_{\text {cumate }}-$ podJ, $\mathrm{P}_{\text {xyl }}-\operatorname{ctr} A \Delta 3 \Omega$

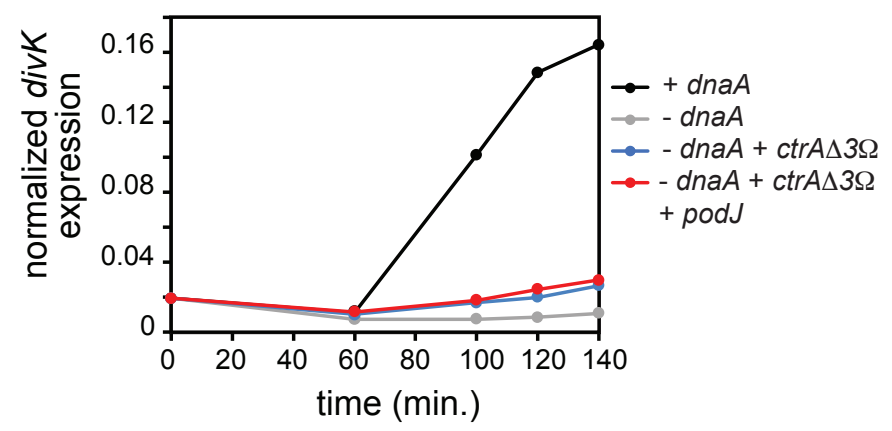

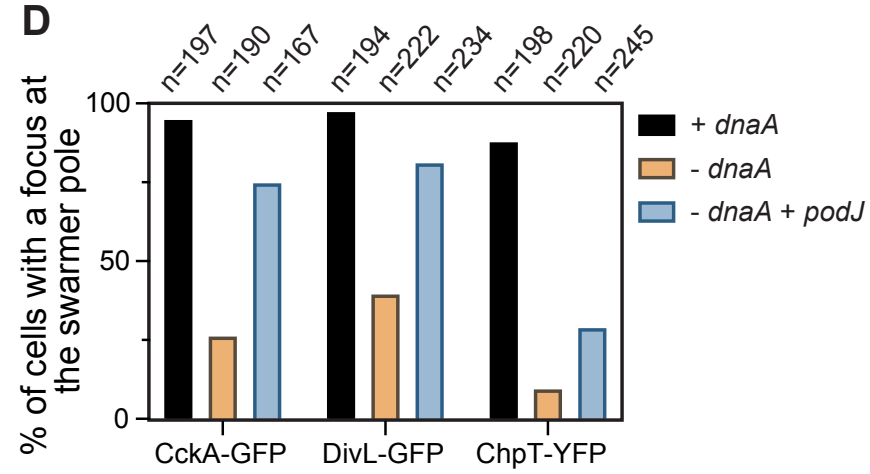

E

CckA-GFP, $\mathrm{P}_{\text {lac }}-$ dnaA, $\mathrm{P}_{\text {cumate }}-$ podJ

$\begin{array}{lll}+\operatorname{dna} A & -d n a A & -d n a A \\ & +p o d J\end{array}$

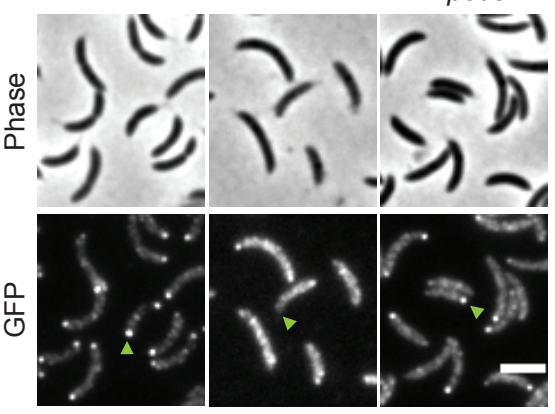

F DivL-GFP, $\mathrm{P}_{\text {lac }}-$ dnaA, $\mathrm{P}_{\text {cumate }}-$ podJ

\begin{tabular}{lll}
\hline$+d n a A$ & $-d n a A$ & $-d n a A$ \\
& $+p o d J$
\end{tabular}

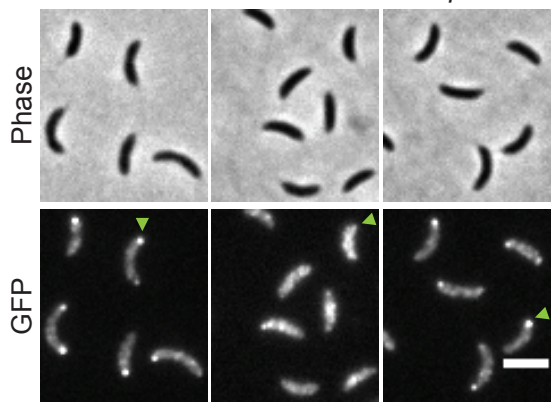

G

ChpT-YFP, $\mathrm{P}_{\text {lac }}$-dnaA, $\mathrm{P}_{\text {cumate }}$-podJ

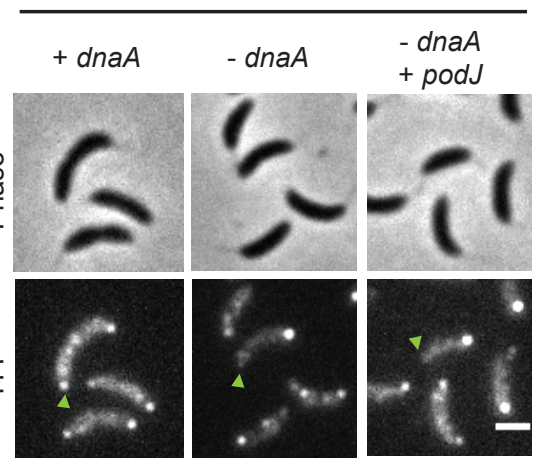


bioRxiv preprint doi: https://doi.org/10.1101/2021.03.08.434431; this version posted March 8, 2021. The copyright holder for this preprint (which was not certified by peer review) is the author/funder, who has granted bioRxiv a license to display the preprint in perpetuity. It is madFigure 2

\section{A}

$\mathrm{P}_{\text {lac }}-d n a A, \mathrm{P}_{x y 1}-d n a A(R 357 A)$

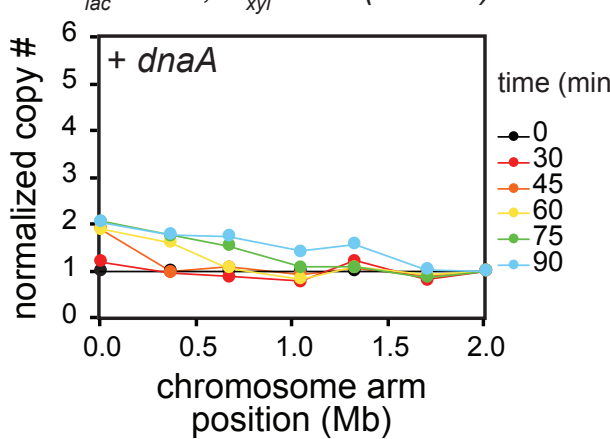

B

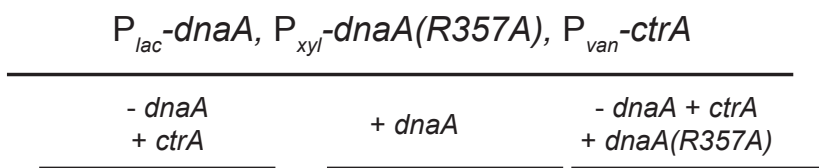

min.: $\quad 0 \overline{60100120140160} \quad 0 \overline{60100120140160} \overline{60100120140160}$

CtrA

RpoA
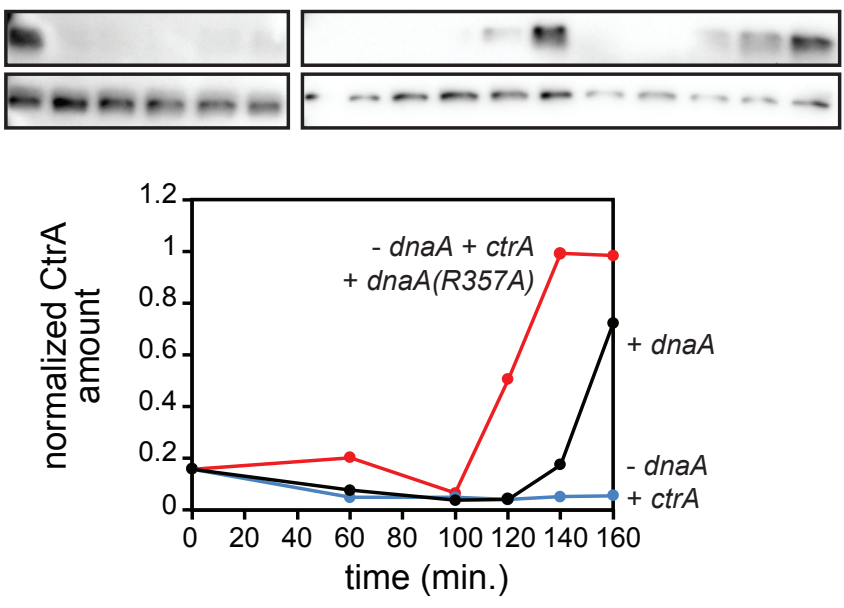

$\mathrm{P}_{\text {lac }}-d n a A, \mathrm{P}_{x y \mid}-d n a A(R 357 A), \mathrm{P}_{\text {van }}-c t r A$

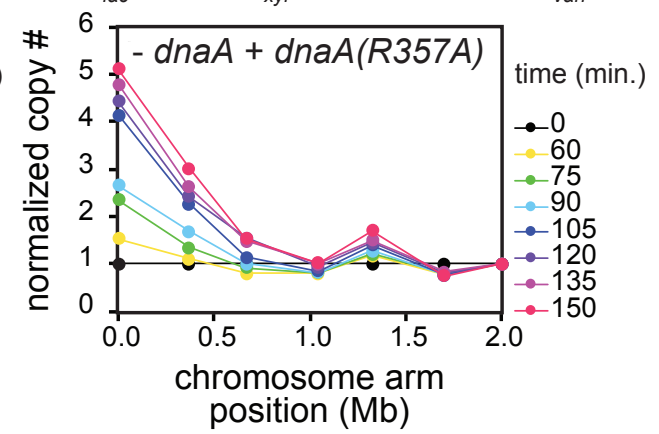

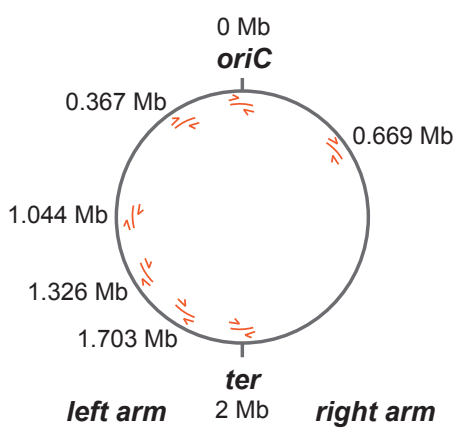

C
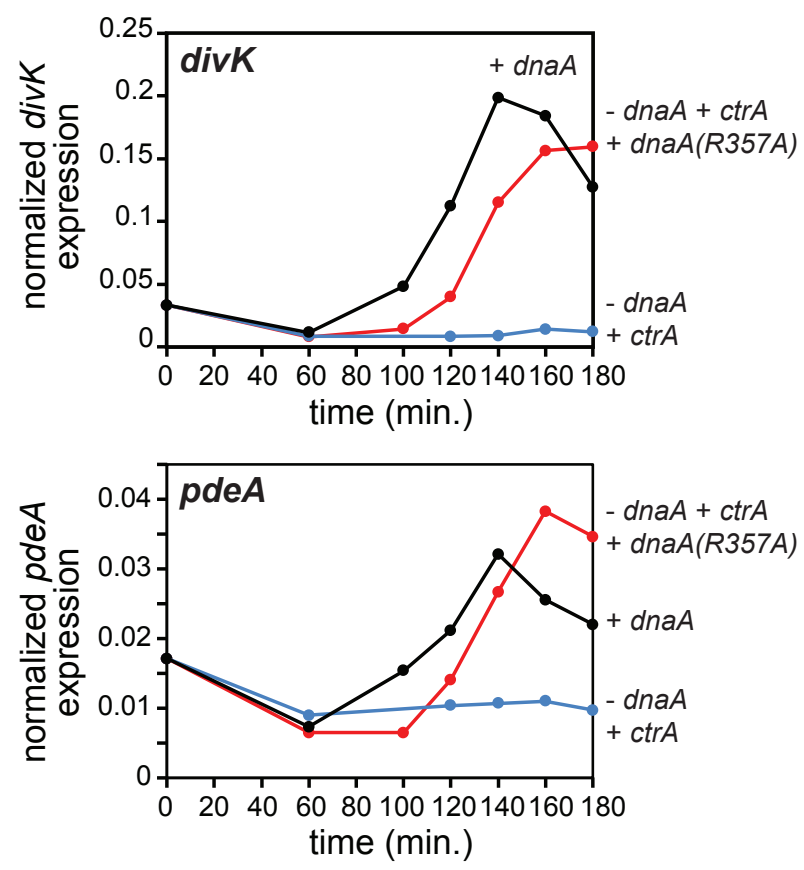
bioRxiv preprint doi: https://doi.org/10.1101/2021.03.08.434431; this version posted March 8, 2021. The copyright holder for this preprint (which was not certified by peer review) is the author/funder, who has granted bioRxiv a license to display the preprint in perpetuity. It is made available under aCC-BY-NC-ND 4.0 International license.

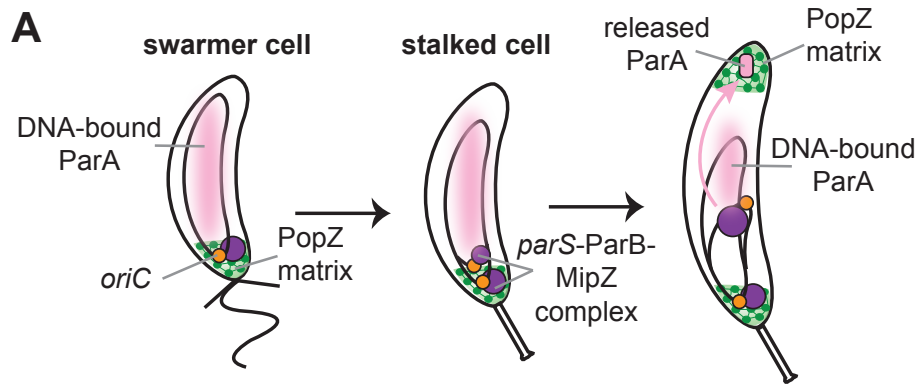

B
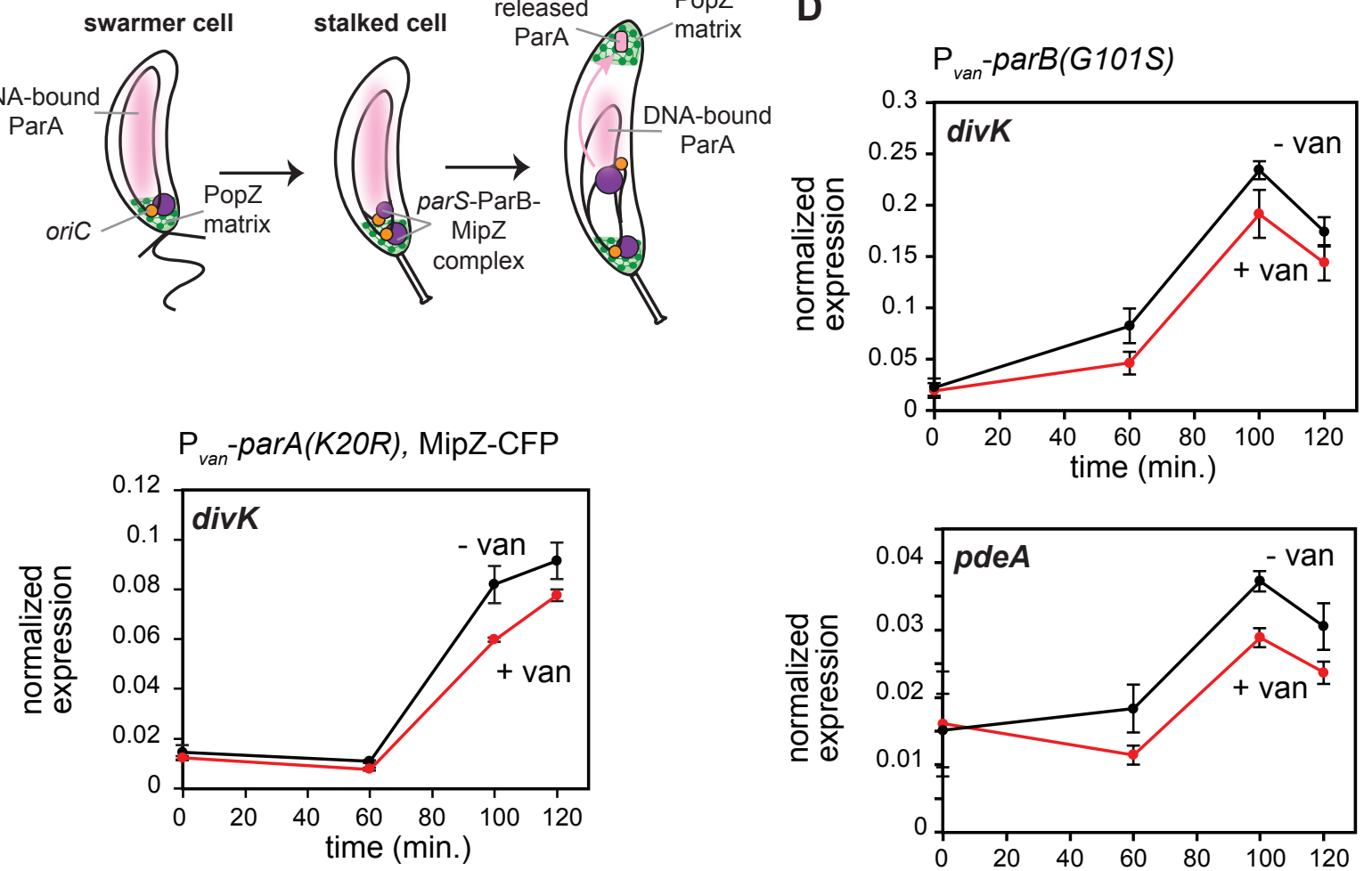

$$
P_{\text {van }}-\operatorname{parB}(\mathrm{G} 101 S)
$$
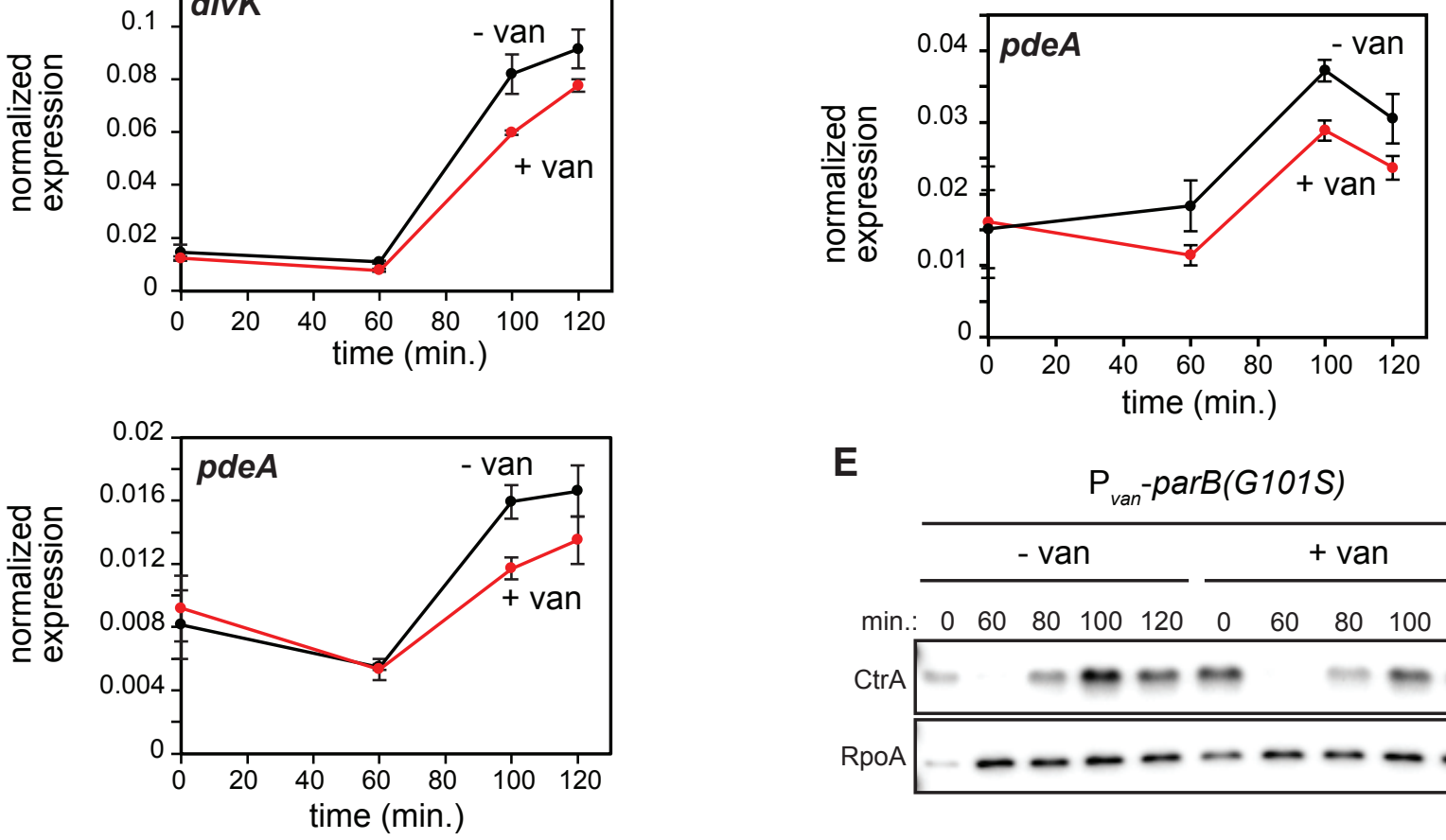

E

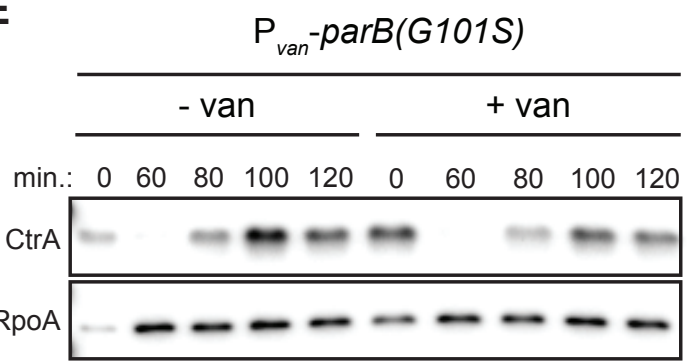

C
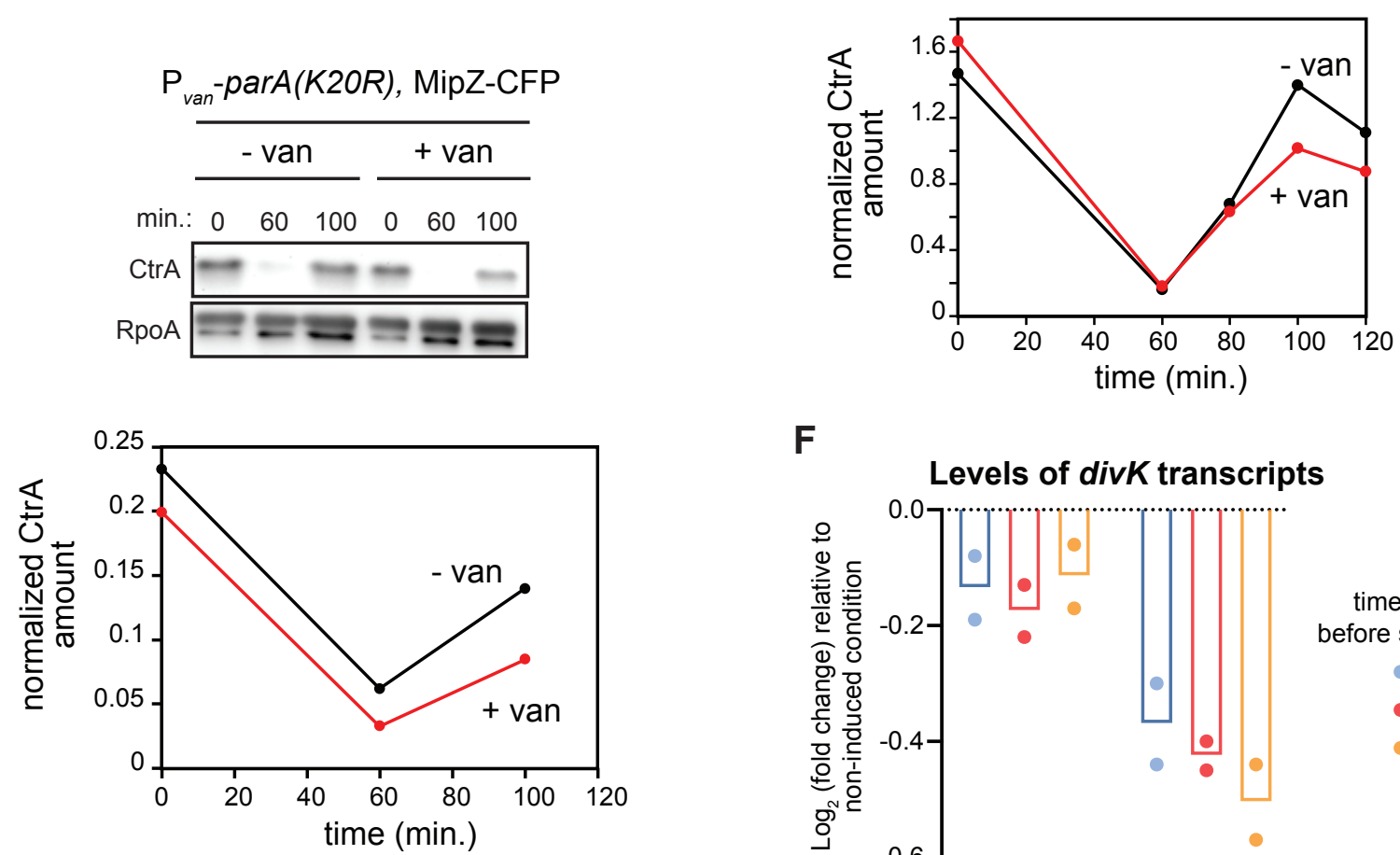

$\mathbf{F}$

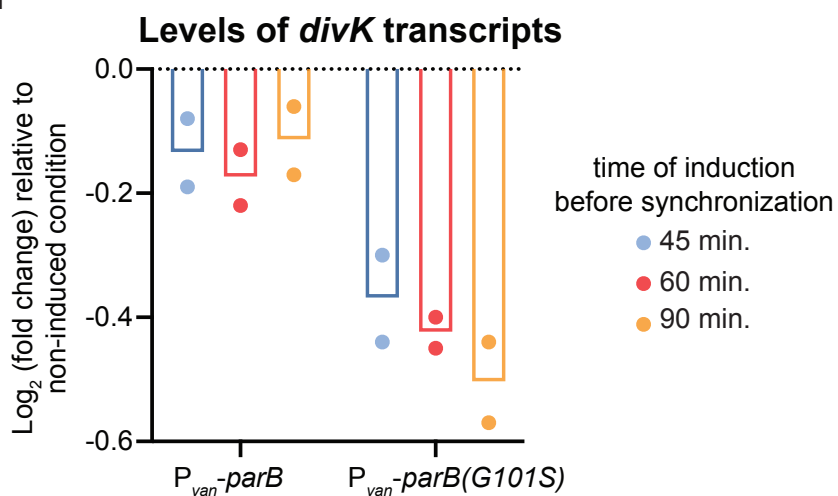


bioRxiv preprint doi: https://doi.org/10.1101/2021.03.08 434431; this version posted March 8, 2021. The copyright holder for this preprint

(which was not certified by peer review) is the author/funder, who has granted bioRxiv a license to display the preprint in perpetuity. It is madeFigure 4 available under aCC-BY-NC-ND 4.0 International license.

A

ChpTsfGFP

60

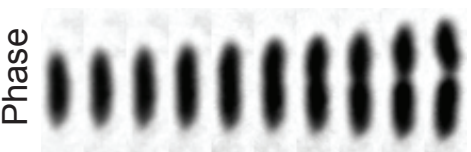

:Liiliiiliti

CckA-GFP, $\mathrm{P}_{\text {van }}$-parA(K20R)

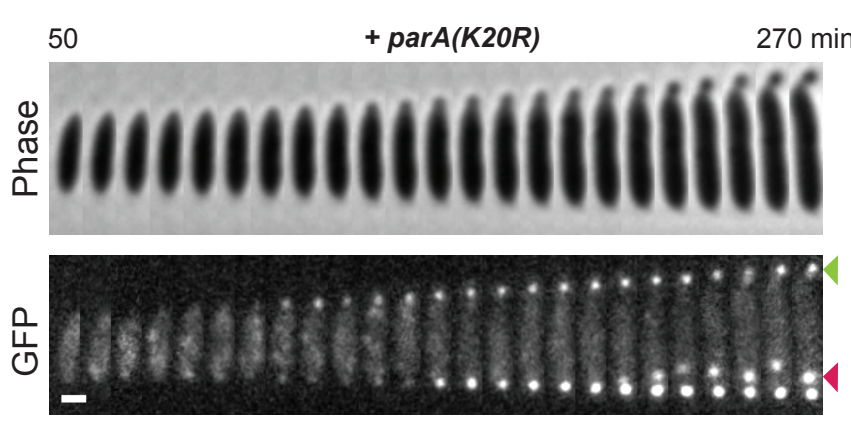

ChpTsfGFP, $P_{\text {van }}-$ parA

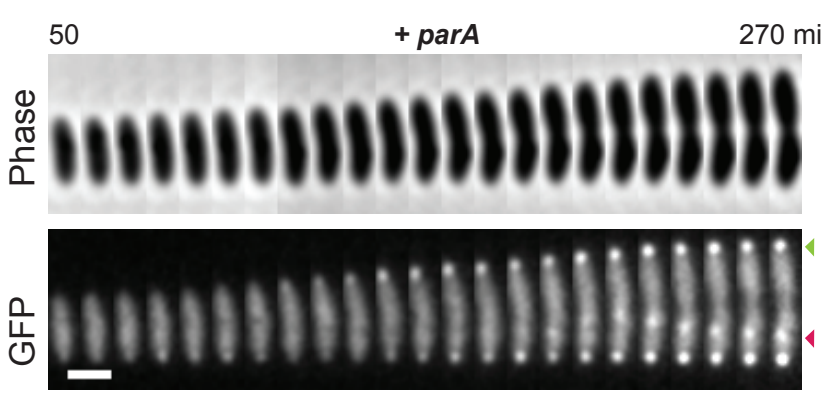

B $\%$ of cells with a focus at

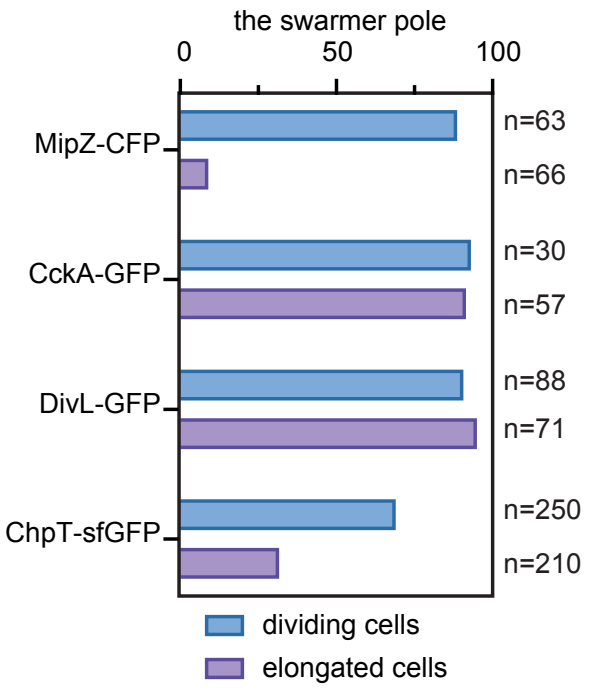

C

MipZ-CFP, $\mathrm{P}_{\text {van }}-$ parA(K20R)
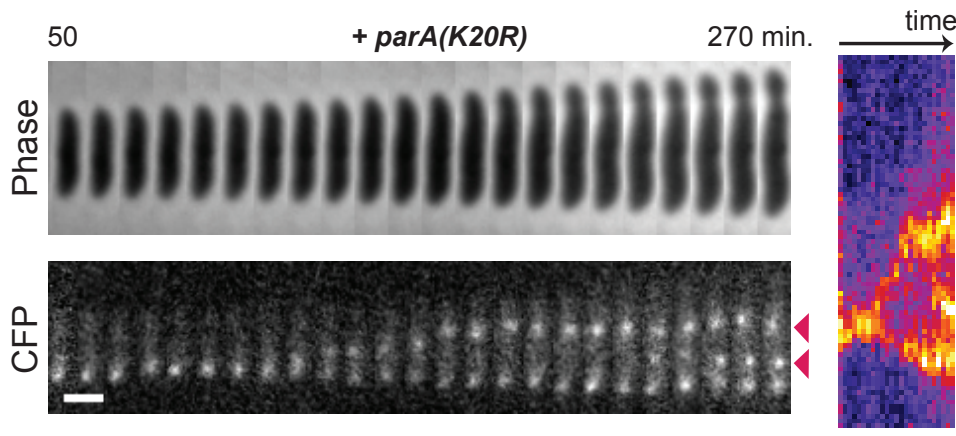

E ChpTsfGFP, $P_{\text {van }}-p a r A(K 20 R)$

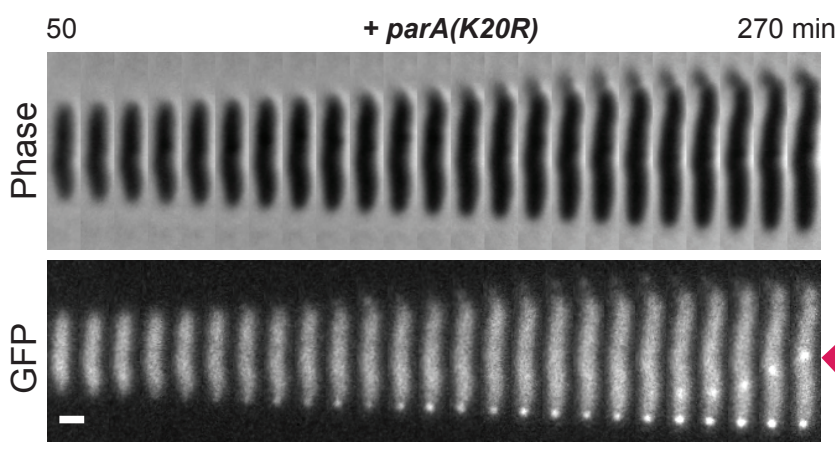

G $\%$ of cells with a focus at the swarmer pole
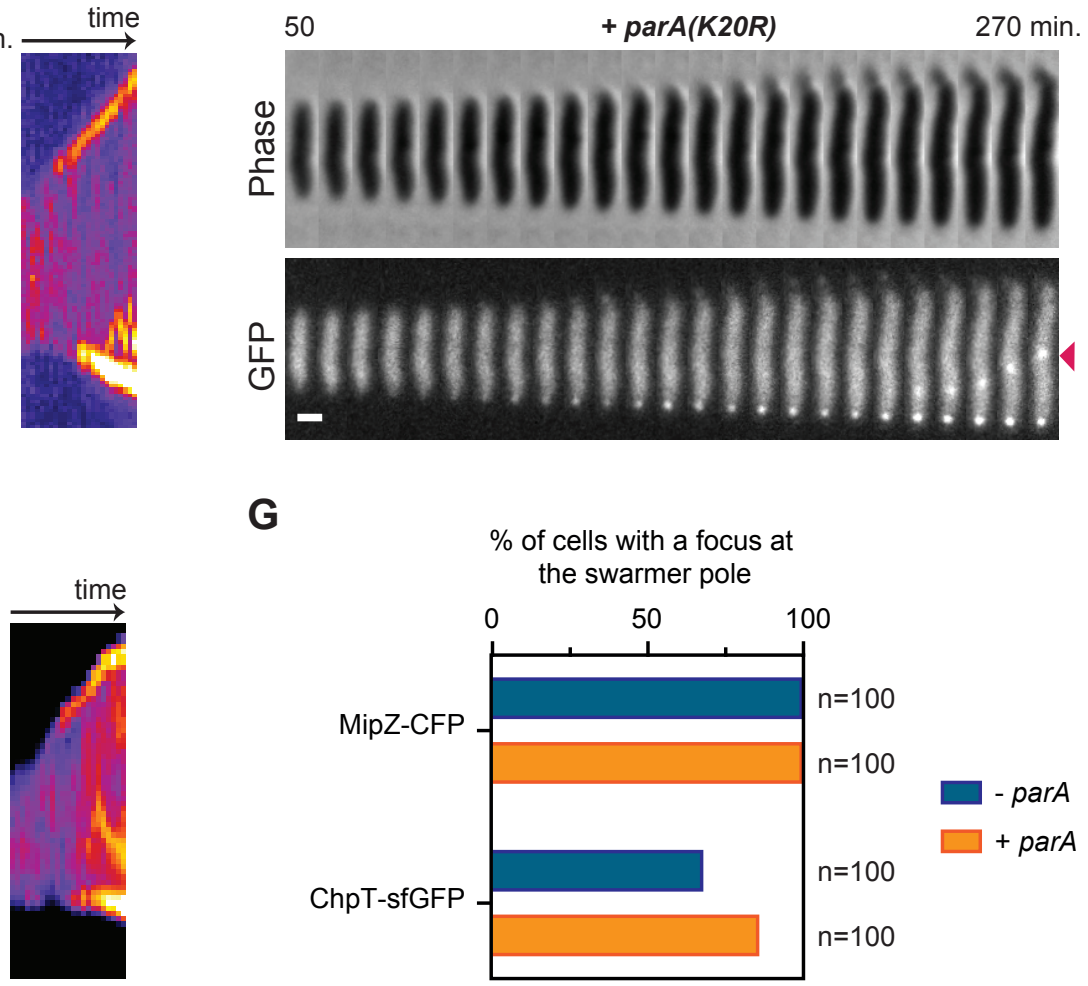
bioRxiv preprint doi: https://doi.org/10.1101/2021.03.08.434431; this version posted March 8, 2021. The copyright holder for this preprint (which was not certified by peer review) is the author/funder, who has granted bioRxiv a license to display the preprint in perpetuity. It is made available under aCC-BY-NC-ND 4.0 International license.

A

CckA-GFP, $\mathrm{P}_{\text {van }}-$ parA(K20R), $\mathrm{P}_{x y l}-$ popZ $\mathrm{t}=190$ min. post synchronization

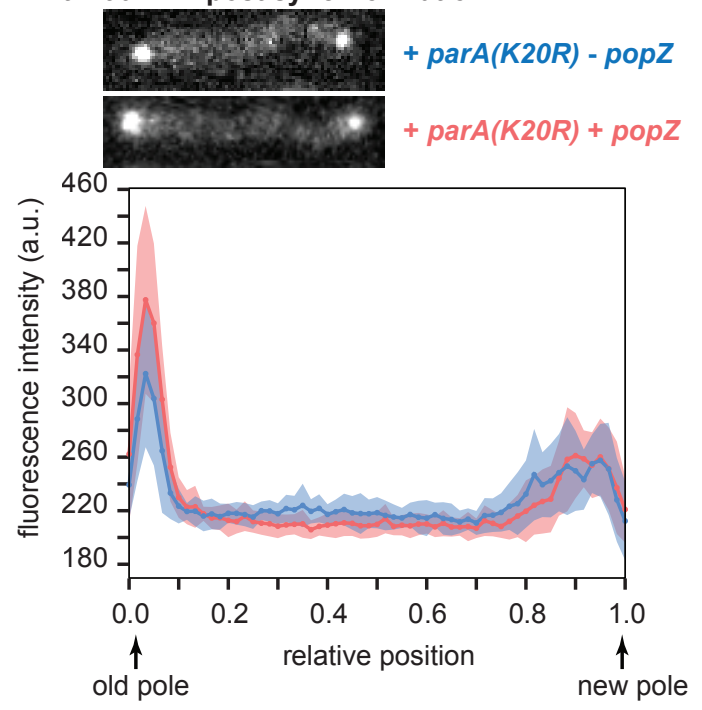

C

ChpT-YFP, $\mathrm{P}_{\text {van }}-$ parA(K20R)-CFP

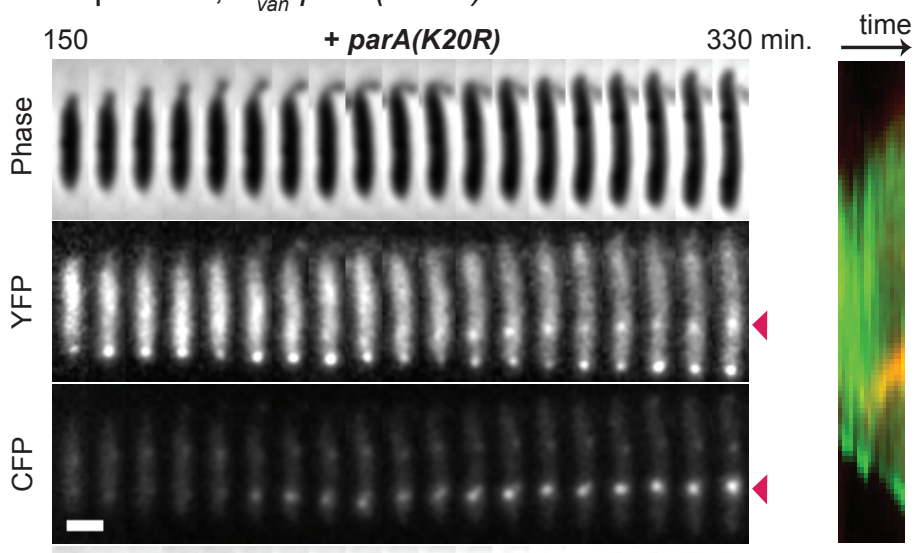

${ }_{0}^{\frac{\pi}{0}}$

$\mathbf{E}$

$\mathrm{P}_{\text {van }}-$ parA(K20R), $\mathrm{P}_{x y \mid}-$ chpT-sfGFP

$30+\operatorname{parA}(K 20 R)+\operatorname{chpT}$-sfGFP

$270 \mathrm{~min}$
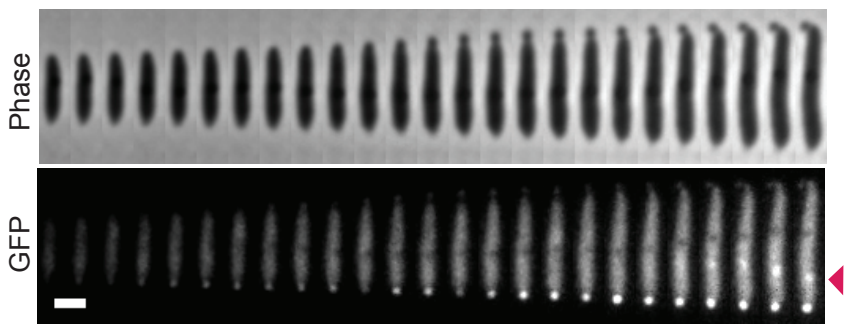

B

ChpT-sfGFP, $\mathrm{P}_{\text {van }}-$ parA(K20R), $\mathrm{P}_{x y 1}-p o p Z$ $\mathrm{t}=190 \mathrm{~min}$. post synchronization
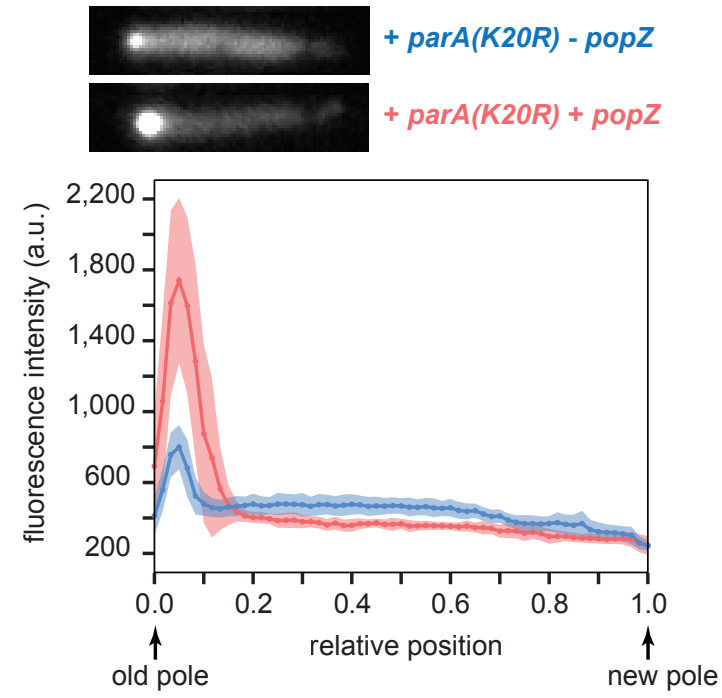

D

ChpT-T25

ChpT $_{\text {DHP }}-\mathrm{T} 25$

T25-CckA ${ }_{H K}$

ChpT $^{*}-\mathrm{T} 25$

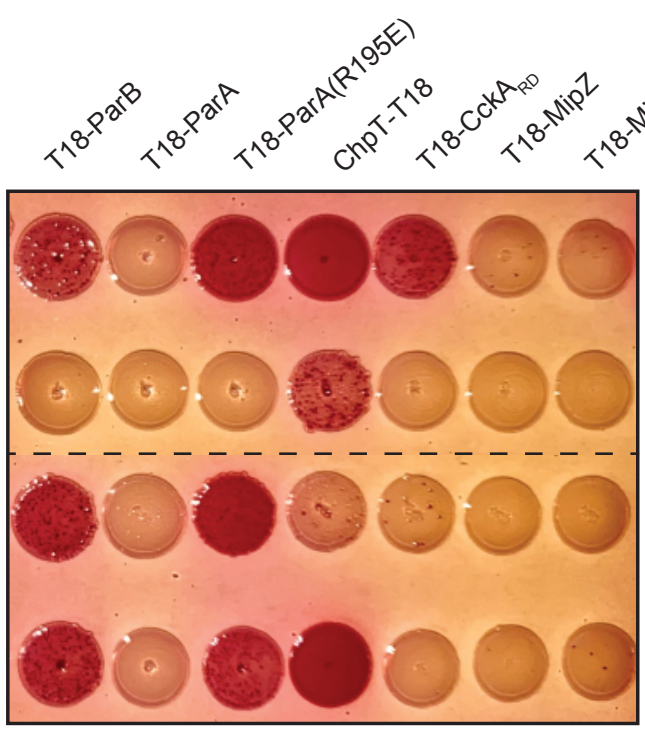

F

$\mathrm{P}_{\text {van }}-\operatorname{parA}(K 20 R), \mathrm{P}_{x y 1}-c h p T^{*}-s f G F P$

30

$+\operatorname{parA}(K 20 R)+\operatorname{chpT}^{*_{-}-\text {sfGFP }}$

$270 \mathrm{~min}$

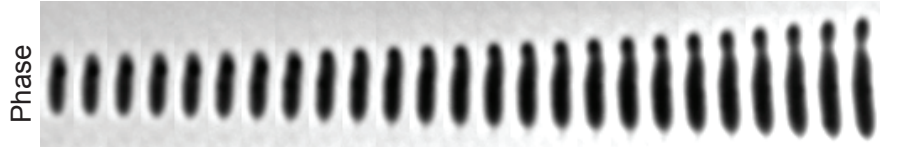

品

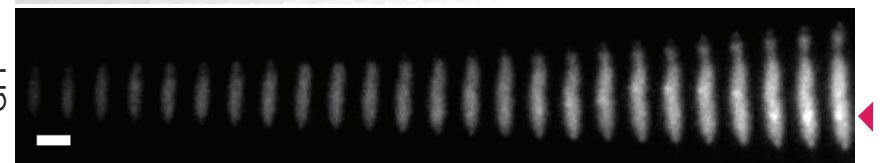


bioRxiv preprint doi: https://doi.org/10.1101/2021.03.08.434431; this version posted March 8, 2021. The copyright holder for this preprint (which was not certified by peer review) is the author/funder, who has granted bioRxiv a license to display the preprint in perpetuity. It is made available under aCC-BY-NC-ND 4.0 International license.
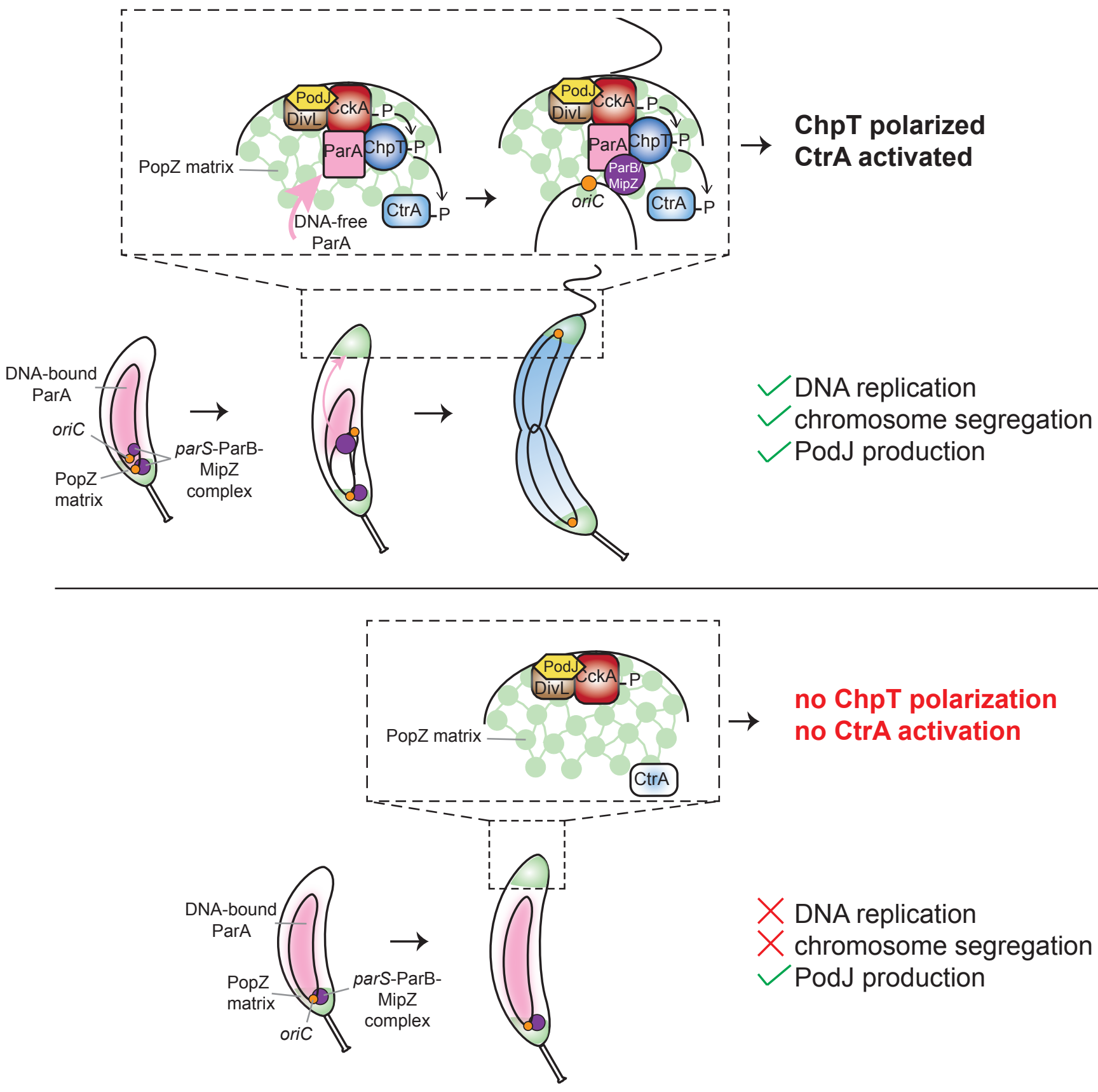

no ChpT polarization

no CtrA activation

X DNA replication

X chromosome segregation

$\checkmark$ PodJ production 
bioRxiv preprint doi: https://doi.org/10.1101/2021.03.08.434431; this version posted March 8, 2021. The copyright holder for this preprint (which was not certified by peer review) is the author/funder, who has granted bioRxiv a license to display the preprint in perpetuity. It is made 9 gre S1
avaBable under aCC-BY-NC-ND 4.0 International license.

A

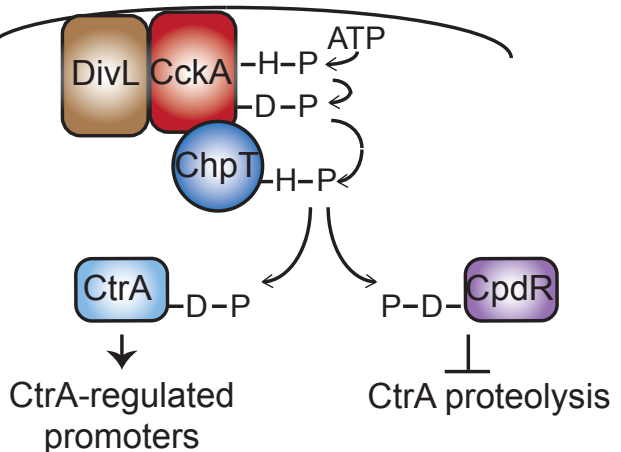

$\mathrm{P}_{\text {lac }}-$ dnaA, $\mathrm{P}_{\text {cumate }}-$ podJ, $\mathrm{P}_{\text {xyl }}-$ ctrA $\quad 0,40,100,120 \mathrm{~min}$.
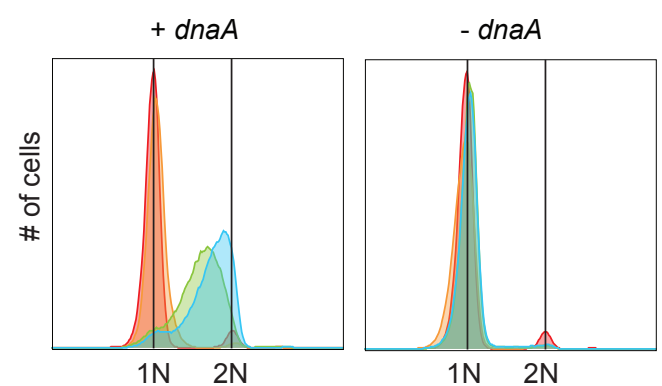

$1 \mathrm{~N} \quad 2 \mathrm{~N}$

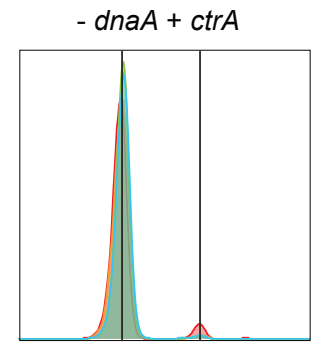

$1 \mathrm{~N} \quad 2 \mathrm{~N}$
- dna $A+c t r A+$ podJ

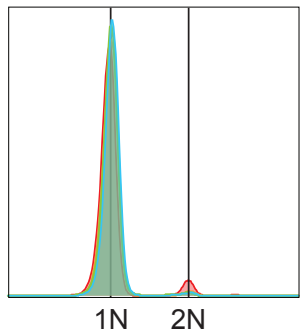

C

$\mathrm{P}_{\text {lac }}-d n a A, \mathrm{P}_{\text {cumate }}-$ podJ, $\mathrm{P}_{x y \mid}-c t r A \Delta 3 \Omega$

$\longrightarrow \quad+\operatorname{dnaA}$

$\rightarrow-d n a A+\operatorname{ctr} A \Delta 3 \Omega$

$\rightarrow-d n a A+\operatorname{ctr} A \Delta 3 \Omega+$ podJ

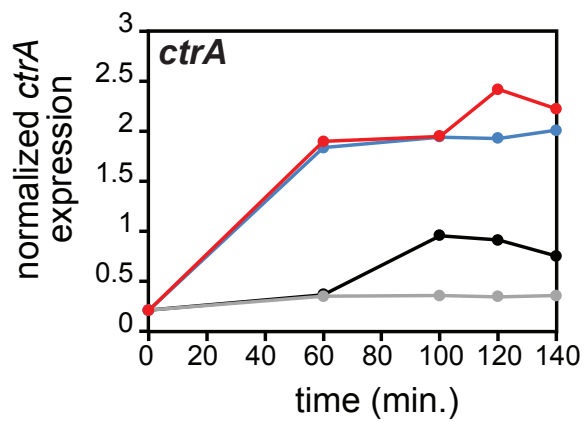

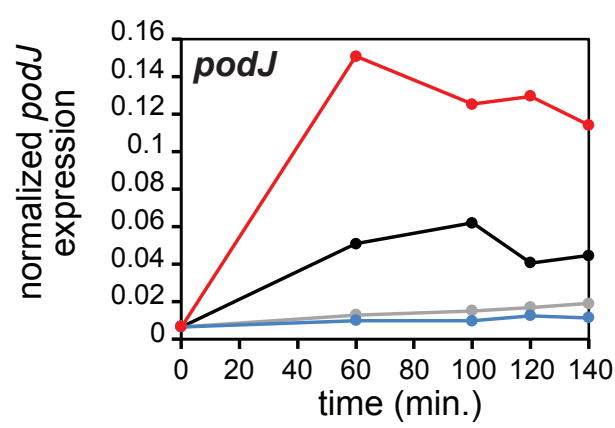

D
$\mathrm{P}_{\text {lac }}-$ dnaA, $\mathrm{P}_{\text {cumate }}-$ podJ, $\mathrm{P}_{x y \mid}-c t r A \Delta 3 \Omega$ - dna $A \quad-d n a A+\operatorname{ctr} A \Delta 3 \Omega$

$\min .:$

$0 \quad 60100 \quad 120140 \quad 60 \quad 100120140$

$\mathrm{CtrA} \triangle 3 \Omega$

RpoA

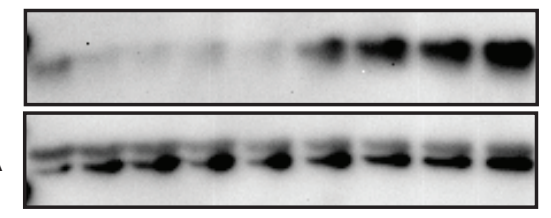

E

$$
\begin{aligned}
& \mathrm{P}_{l a c}-d n a A, \mathrm{P}_{x y l}-g c r A-3 X \text { flag, } \mathrm{P}_{v a n}-c t r A \\
& + \text { dnaA } \frac{-d n a A+c t r A}{+}+\text { ctrA + gcrA-3Xflag }
\end{aligned}
$$

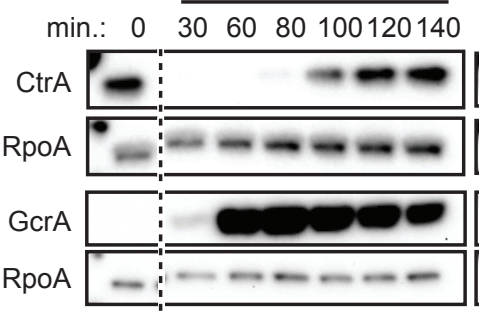

$\rightarrow+\operatorname{dna} A$

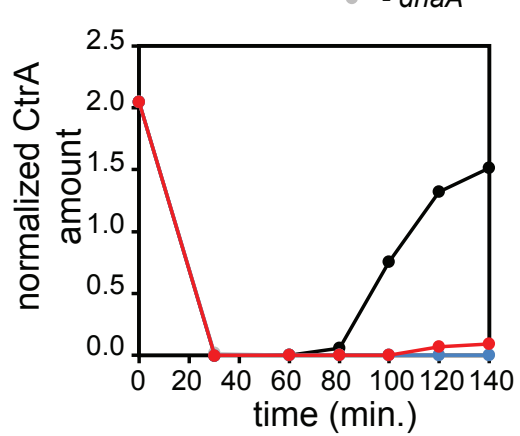

$\rightarrow-d n a A+$ ctrA + gcrA-3Xflag

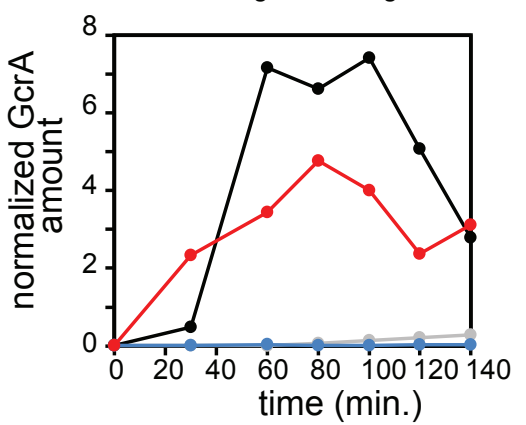

H

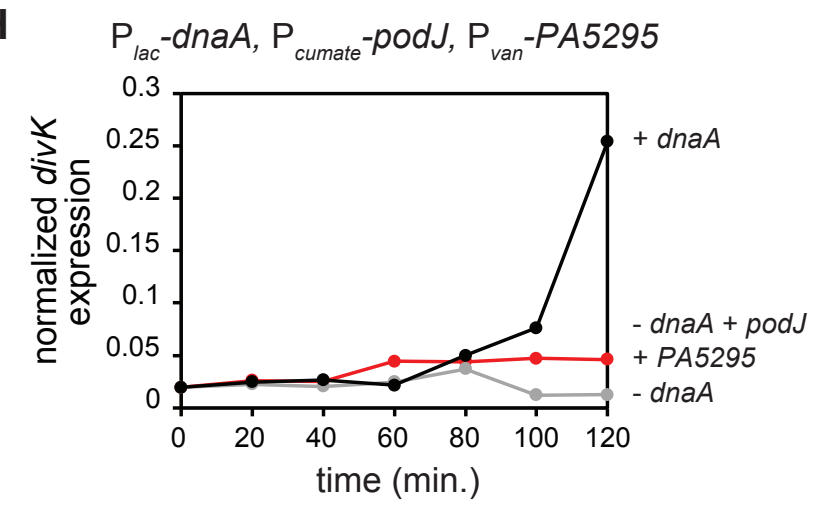

G

F

$\mathrm{P}_{\text {lac }}-$ dnaA, $\mathrm{P}_{x y \mid}-$ gcrA-3Xflag, $\mathrm{P}_{\text {van }}-\operatorname{ctr} A \Delta 3 \Omega$
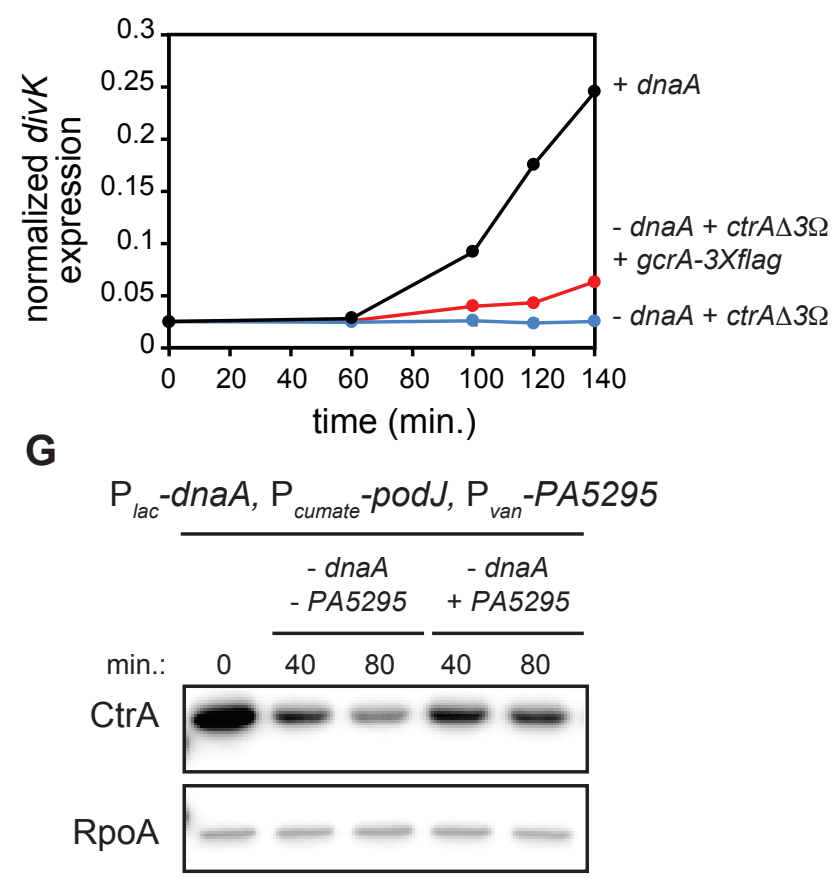
bioRxiv preprint doi: https://doi.org/10.1101/2021.03.08.434431; this version posted March 8, 2021. The copyright holder for this preprint (which was not certified by peer review) is the author/funder, who has granted bioRxiv a license to display the preprint in perpetuity. It is made
available under aCC-BY-NC-ND 4.0 International license.

A

$\mathrm{P}_{l a c}-d n a A, \mathrm{P}_{x y \mid}-d n a A(R 357 A), \mathrm{P}_{\mathrm{van}}-c t r A$

$+d n a A$

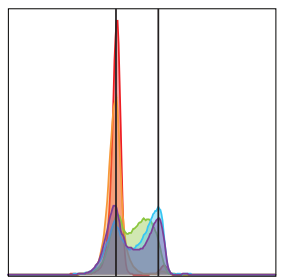

$1 \mathrm{~N} \quad 2 \mathrm{~N}$ $-d n a A+c t r A$

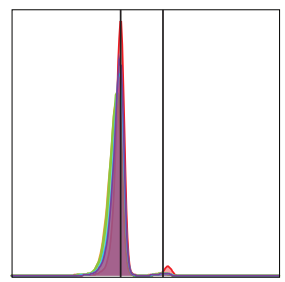

$1 \mathrm{~N} \quad 2 \mathrm{~N}$
- dna $A+$ ctrA $+\operatorname{dnaA}(R 357 A)$

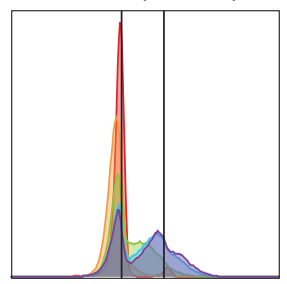

$1 \mathrm{~N} 2 \mathrm{~N}$

B

$0,60,120,160,180 \mathrm{~min}$.

C

$$
\mathrm{P}_{l a c}-d n a A, \mathrm{P}_{x y y}-d n a A(R 357 A), \mathrm{P}_{\mathrm{van}}-c t r A
$$

$$
+d n a A
$$

\begin{tabular}{|c|c|c|c|c|c|}
\hline $\min .:$ & 0 & 40 & $\begin{array}{lll}60 & 80 & 100120140\end{array}$ & 0 & 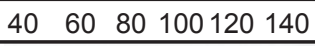 \\
\hline GcrA & & & 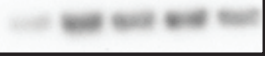 & & 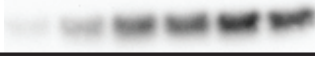 \\
\hline RpoA & 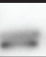 & - & $-\infty-\infty$ & $=$ & 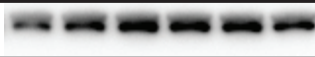 \\
\hline
\end{tabular}

- dnaA + ctrA + dnaA(R357A)

$$
\mathrm{P}_{l a c}-d n a A, \mathrm{P}_{x y l}-d n a A(R 357 A)
$$

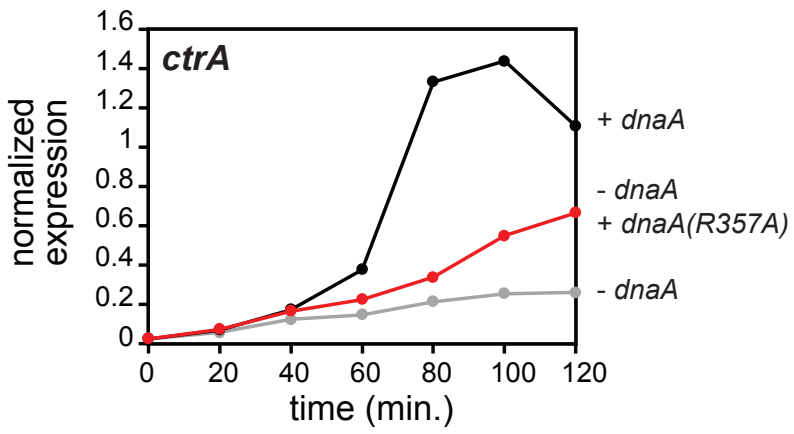

D

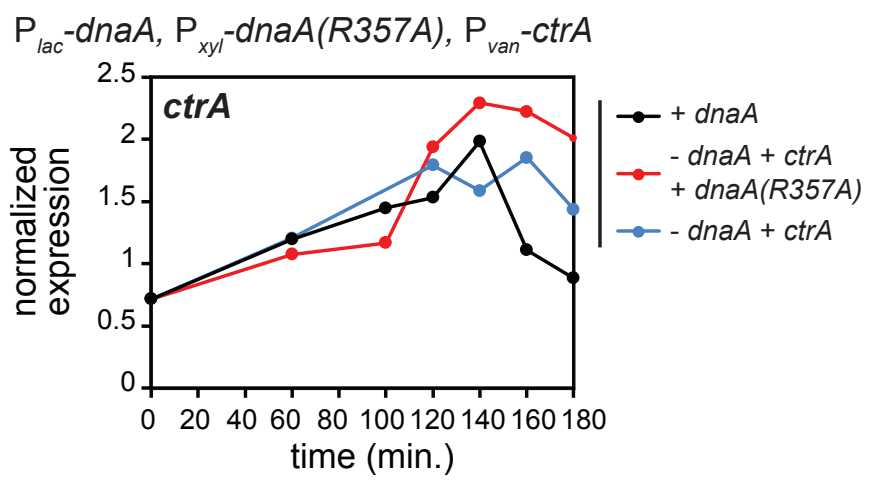


bioRxiv preprint doi: https://doi.org/10.1101/2021.03.08.434431; this version posted March 8, 2021. The copyright holder for this preprint (which was not certified by peer review) is the author/funder, who has granted bioRxiv a license to display the preprint in perpetuity. It is mapte

A

$\mathrm{P}_{\text {van }}-\operatorname{parA}(K 20 R)$, MipZ-CFP

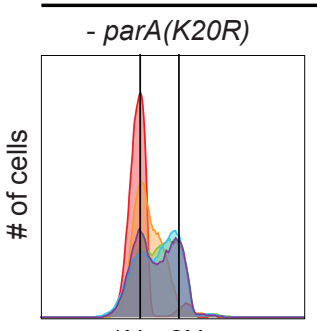

$1 \mathrm{~N} 2 \mathrm{~N}$
$+\operatorname{parA}(K 20 R)$

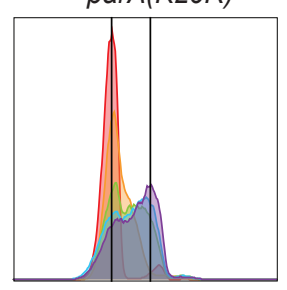

$1 \mathrm{~N} 2 \mathrm{~N}$

B

0, 60, 100, 120, 140 min.

$\mathrm{P}_{\text {van }}-\operatorname{parB}(\mathrm{G} 101 \mathrm{~S})$

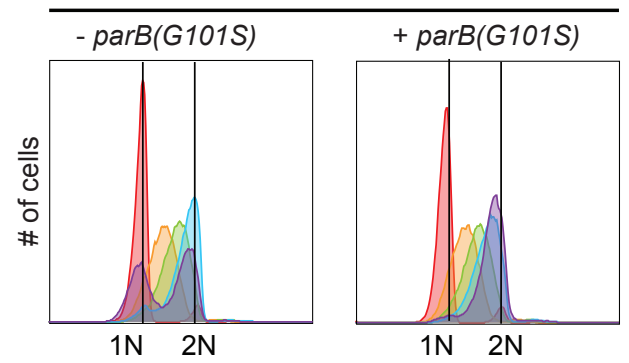

0, 60, 80, 100, 120 min.

\section{C}

MipZ-CFP, $P_{\text {van }}-\operatorname{parB(G101S)}$

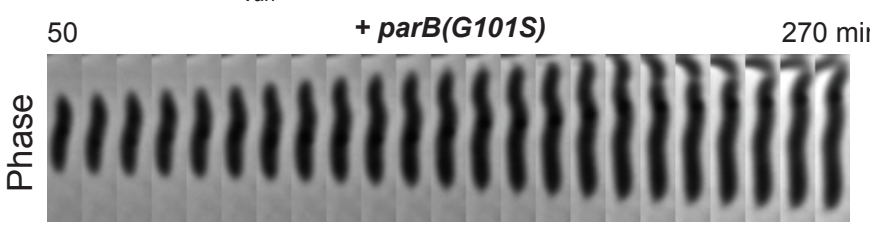

$\frac{0}{0}$
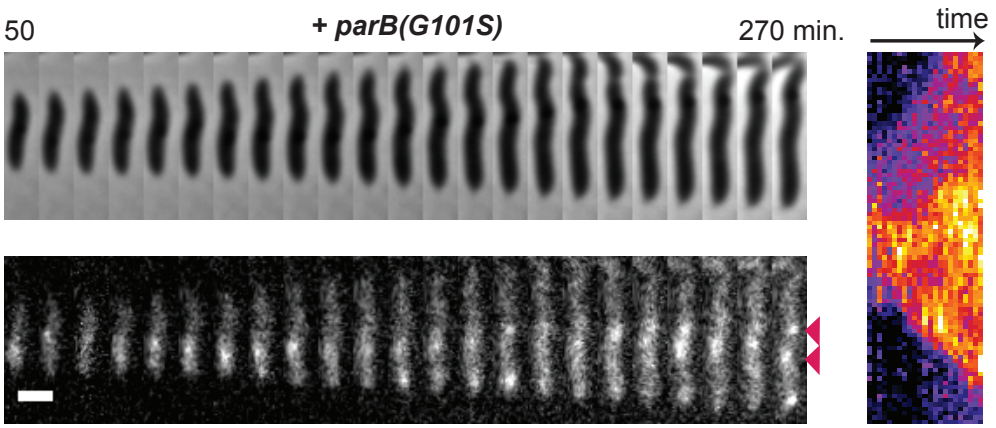
bioRxiv preprint doi: https://doi.org/10.1101/2021.03.08.434431; this version posted March 8, 2021. The copyright holder for this preprint (which was not certified by peer review) is the author/funder, who has granted bioRxiv a license to display the preprint in perpetuity. It is malpgure $\mathbf{S 4}$ available under aCC-BY-NC-ND 4.0 International license.

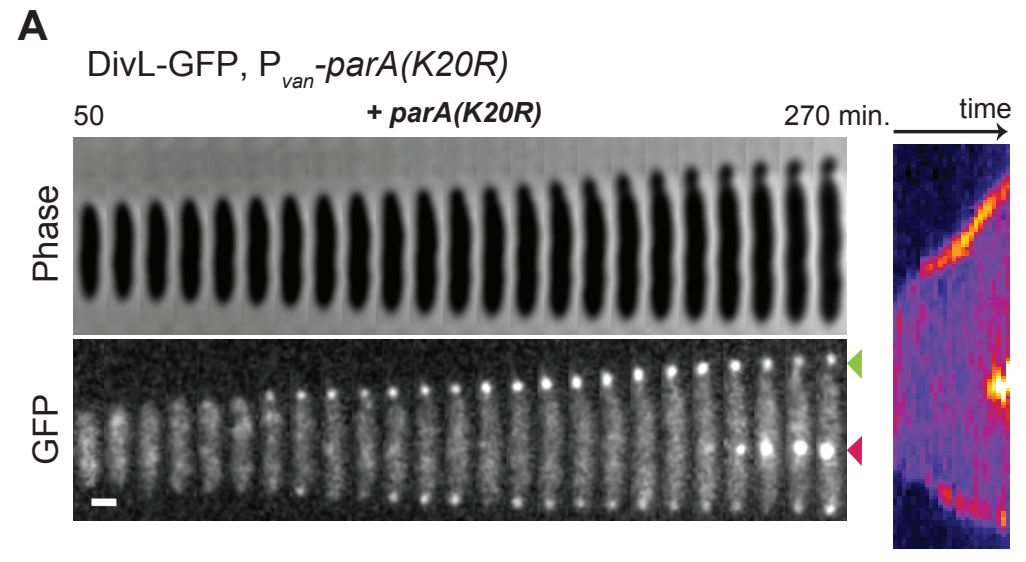

B

MipZ-CFP, $\mathrm{P}_{\text {van }}-$ parA
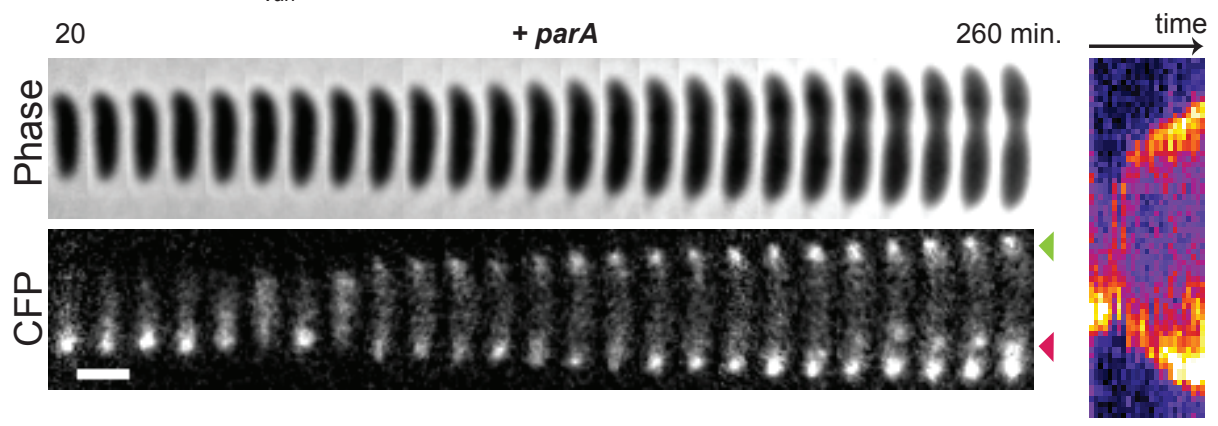

C

PopZ-YFP, $\mathrm{P}_{\text {van }}-\operatorname{parA}(K 20 R)$

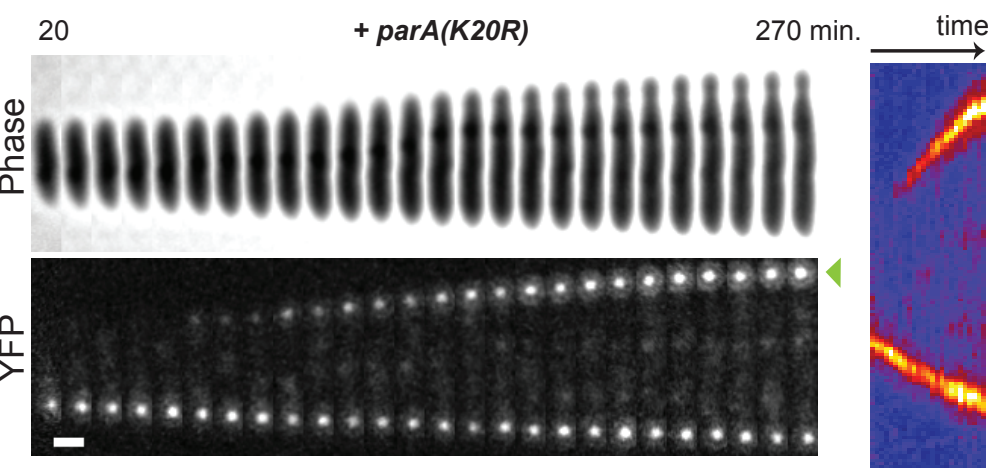


bioRxiv preprint doi: https://doi.org/10.1101/2021.03.08.434431; this version posted March 8, 2021. The copyright holder for this preprint (which was not certified by peer review) is the author/funder, who has granted bioRxiv a license to display the preprint in perpetuity. It is megigure $\mathbf{S 5}$
available under aCC-BY-NC-ND 4.0 International license.

A

CckA-GFP, $P_{v a n}-p a r A(K 20 R), P_{x y 1}-p o p Z$

$+\operatorname{parA}(K 20 R)$ - popZ

$190 \mathrm{~min}$.

$+\operatorname{parA}(K 20 R)+\operatorname{popZ}$

60

$\downarrow$ $270 \min$

60 $270 \min$
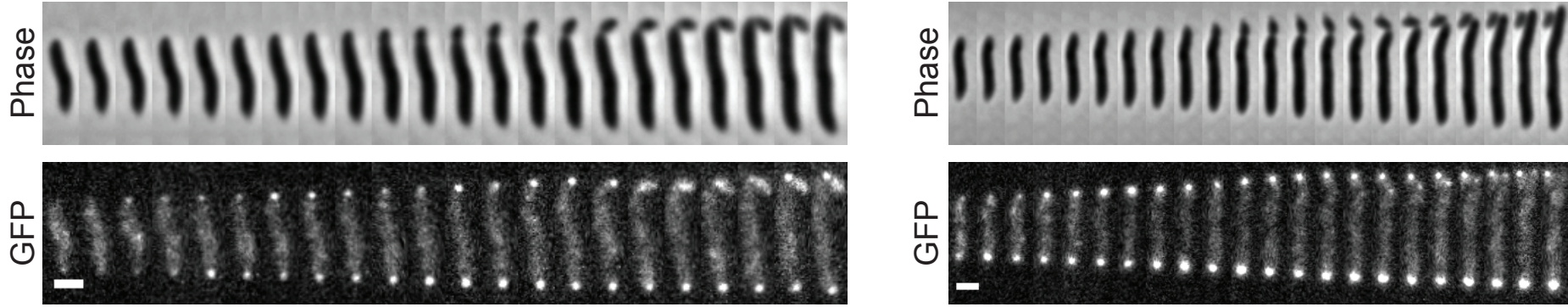

B

ChpTsfGFP, $\mathrm{P}_{\text {van }}-$ parA(K20R), $\mathrm{P}_{x y /}-$ popZ

+ parA $(K 20 R)$ - popZ

60

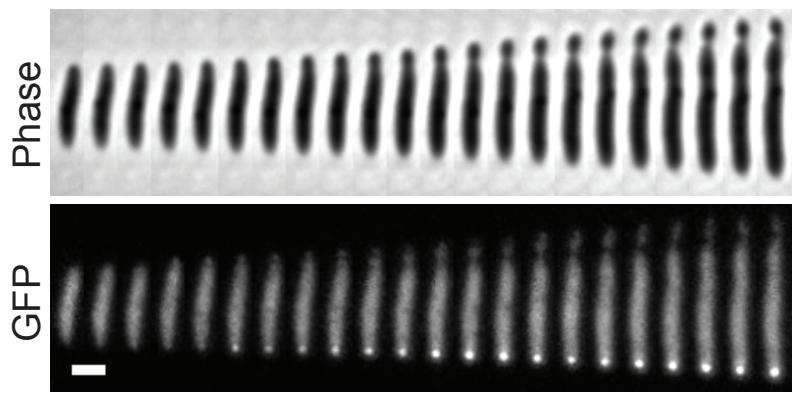

$+p a r A(K 20 R)+p o p Z$

$270 \min$.

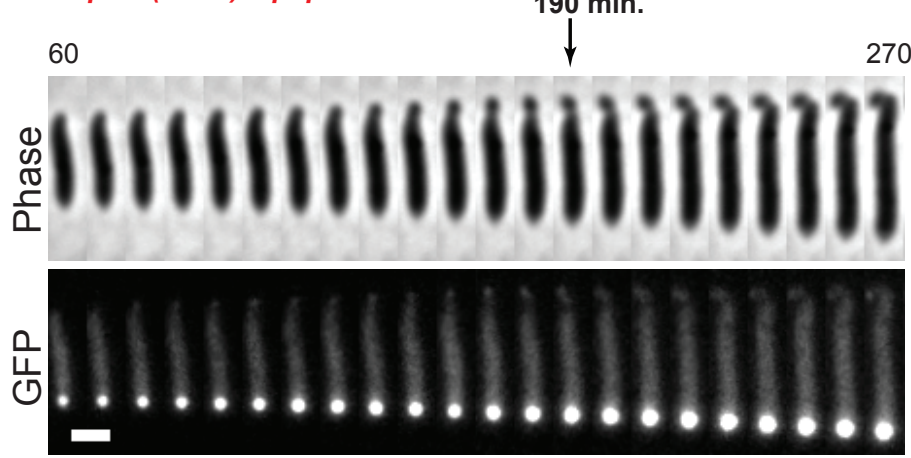

C

YFP-MipZ, $\mathrm{P}_{\text {van }}-$ parA(K20R)-CFP

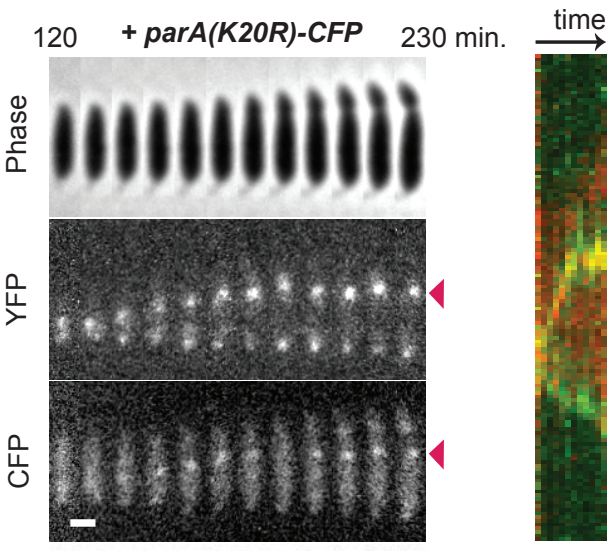

D

ChpT-T25

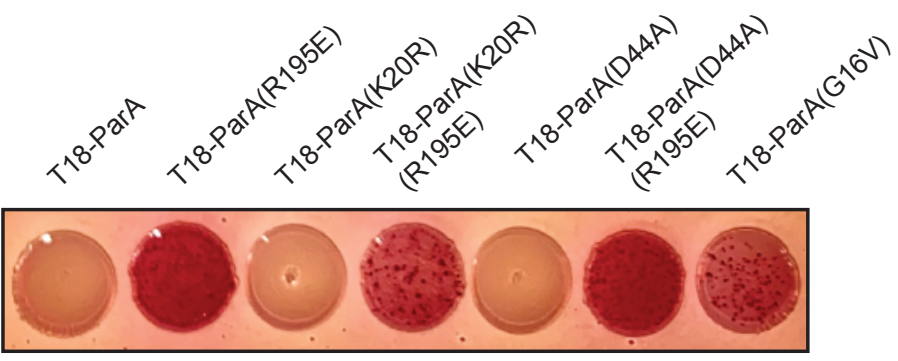

Is:

E

$\mathrm{P}_{\text {van }}-\operatorname{parA}(K 20 R), \mathrm{P}_{x y 1}-\operatorname{chpT}$-sfGFP

$30-$ - parA $(K 20 R)+$ chpT-sfGFP

$170 \mathrm{~min}$.

[IIIIIIIIIIIII)

品

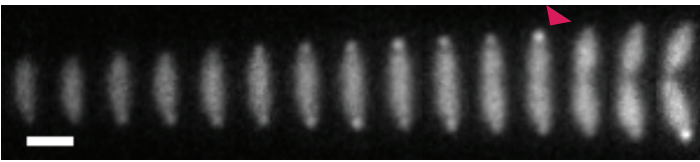

$\mathbf{F}$ $\mathrm{P}_{\text {van }}-\operatorname{parA}(K 20 R), \mathrm{P}_{x y 1}-\operatorname{chp} T^{*}$-sfGFP

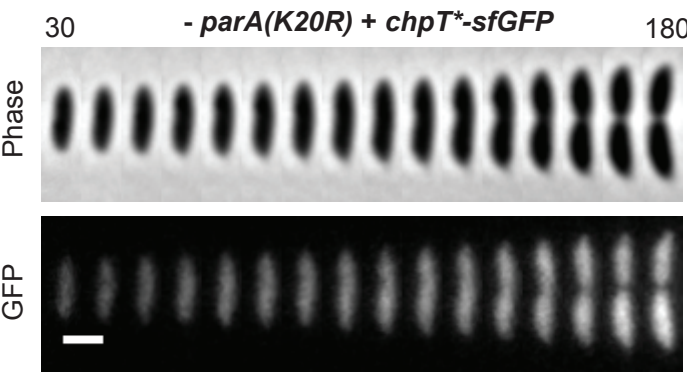

